Prepared for the U.S. Department of Energy under Contract DE-AC05-76RL01830

\title{
GTRI Remote Monitoring System: Training and Operational Needs Assessment Analysis Report
}

Prepared by:

Debra Day, Senior Security Operations Specialist

Sorcha Fox, Instructional Designer

April 2012

Pacific Northwest

NATIONAL LABORATORY

Proudly Operated by Battelle Since 1965 


\title{
DISCLAIMER
}

This report was prepared as an account of work sponsored by an agency of the United States Government. Neither the United States Government nor any agency thereof, nor Battelle Memorial Institute, nor any of their employees, makes any warranty, express or implied, or assumes any legal liability or responsibility for the accuracy, completeness, or usefulness of any information, apparatus, product, or process disclosed, or represents that its use would not infringe privately owned rights. Reference herein to any specific commercial product, process, or service by trade name, trademark, manufacturer, or otherwise does not necessarily constitute or imply its endorsement, recommendation, or favoring by the United States Government or any agency thereof, or Battelle Memorial Institute. The views and opinions of authors expressed herein do not necessarily state or reflect those of the United States Government or any agency thereof.

\author{
PACIFIC NORTHWEST NATIONAL LABORATORY \\ operated by \\ BATTELLE \\ for the \\ UNITED STATES DEPARTMENT OF ENERGY \\ under Contract DE-AC05-76RL01830
}

Printed in the United States of America
Available to DOE and DOE contractors from the Office of Scientific and Technical Information,
P.O. Box 62, Oak Ridge, TN 37831-0062;
ph: (865) 576-8401
fax: $(865)$ 576-5728
email: reports@adonis.osti.gov

\begin{abstract}
Available to the public from the National Technical Information Service, U.S. Department of Commerce, 5285 Port Royal Rd., Springfield, VA 22161 ph: (800) 553-6847 fax: $(703) 605-6900$ email: orders@ntis.fedworld.gov online ordering: http://www.ntis.gov/ordering.htm
\end{abstract}

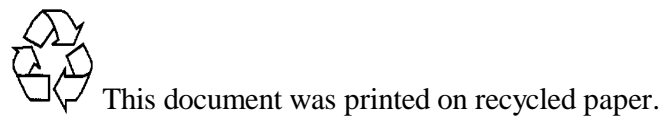




\section{GTRI Remote Monitoring System: Training and Operational Needs Assessment Analysis Report}

Debra Day

Sorcha Fox

April 2012

Prepared for the U.S. Department of Energy under Contract DE-AC05-76RL01830

Pacific Northwest National Laboratory

Richland, Washington 99352 
PNNL-21312 


\section{Table of Contents}

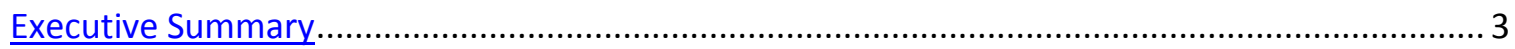

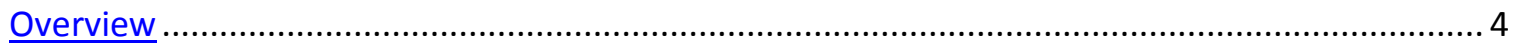

Goals

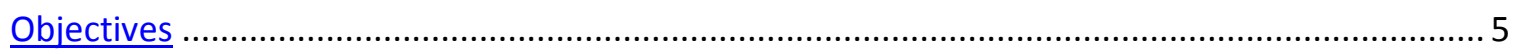

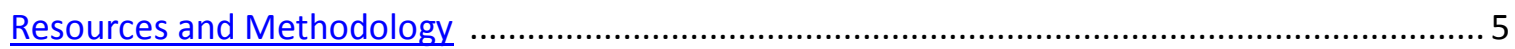

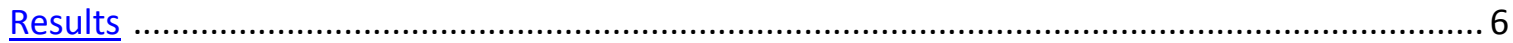

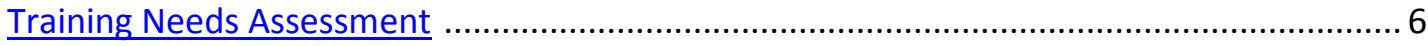

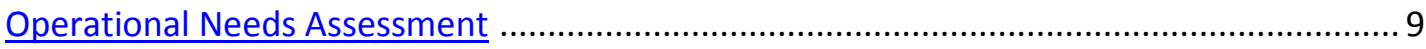

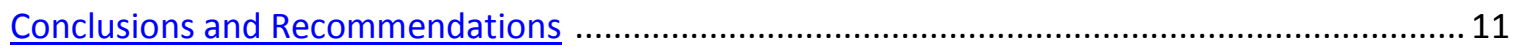

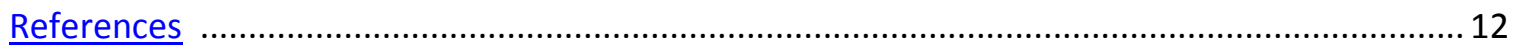

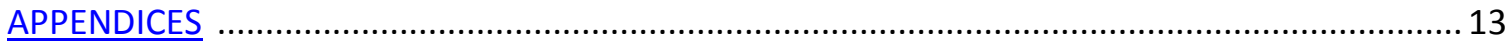

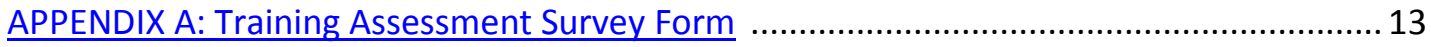

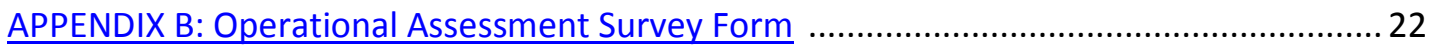

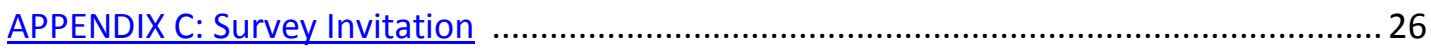

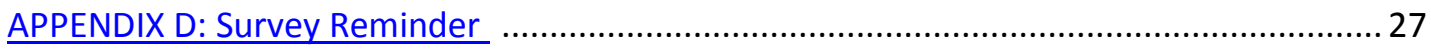

APPENDIX E: Training Needs Assessment Data Tables and Graphs …................................. 28

APPENDIX F: Operational Needs Assessment Data Tables and Graphs ............................... 49

APPENDIX G: Initial Survey Recipient Listing by Location ...................................................5

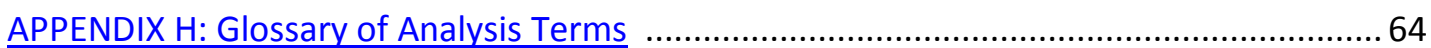




\section{GTRI Remote Monitoring System: Training and Operational Needs Assessment Analysis Report}

\section{Executive Summary}

The mission of the United States Department of Energy (DOE) National Nuclear Security Administrations' (NNSA's) Global Threat Reduction Initiative (GTRI) is to identify, secure, recover and facilitate the disposition of vulnerable nuclear and high-risk radioactive materials around the world that pose a threat to the United States and the international community. The GTRI's unique mission to reduce and protect vulnerable nuclear and radiological materials located at civilian sites worldwide directly addresses recommendations of the $9 / 11$ Commission ${ }^{1}$, and is a vital part of the President's National Security Strategy and the Global Initiative.

The Remote Monitoring System (RMS) is a tool implemented by PNNL and other labs that integrates critical facility alarms at nuclear and radiological sites and supports the GTRI mission. The RMS is a standalone security system that includes radiation and tamper alarms, and CCTV; which can be transmitted securely over the Internet to multiple on-site and off-site locations. It was designed by a third party vendor, Yamasato Fujiwara Higa \& Associates, Inc. (YFH), who also provides RMS installation, training, and support services. Training is provided by YFH engineers during the initial installation visit. Advance notice is provided to sites with recommendations on which key stakeholders should attend the training. Manuals are also e-mailed to the site ahead of time. The training consists of a group demonstration of the software, usually for one to five users and stakeholders. YFH also provides quarterly troubleshooting sessions via WebEx web conferencing, which are typically attended by IT technicians and system administrators.

Through our experiences during installation of the system at 162 sites, plus feedback received from Alarm Response Training course participants, site input to project teams and analysis of trouble calls; indications were that current system training may be lacking and inconsistent. An assessment survey was undertaken to gather information from RMS users across the nation, to evaluate the current level of training and determine what, if any, improvements needed to be made. An additional survey focused on operational ease of the RMS software. Both surveys focused on day-to-day RMS software tasks, as most users are not responsible for operation and maintenance of the entire RMS system architecture. The surveys were initially sent electronically to 245 users at the PNNL GTRI partner sites with RMS installations and achieved a $37.6 \%$ return rate for the training needs survey, and a $17.1 \%$ return rate for the operational needs assessment.

Analysis of the training survey data revealed that $34.6 \%$ of the respondents had not received training or were unsure if they had, despite the fact that vendor engineers provide training at installation of the system. Some respondents commented that their training was "minimal," and "brief, not documented, and nothing in writing." $63.7 \%$ of respondents said they were either not at all prepared or only somewhat prepared to use the RMS software required to effectively operate the system. In addition, some feedback indicated a potential training or supplementary resources need for the overall system architecture, such as: "I feel the RMS monitoring screen and the systems being monitored are too complicated. We have had large numbers of EMS responses to our site because of misinterpretations of the alarm screens by central station operators at the police departments. Simple loss of internet connection is resulting in a full armed response by the EMS team"; "I do not feel well prepared to

\footnotetext{
${ }^{1}$ The 9/11 Commission Report: Final Report of the Commission on Terrorist Attacks upon the United States (New York: Norton 2004).
} 
troubleshoot"; and "The training covered the main alarms but did not cover the workings of the communication between the field hardware and the assessment computer".

As for the operational needs assessment survey, overall satisfaction for the software was in the good range ( 3.5 out of 5). Satisfaction for maintenance procedures and helpdesk assistance was in the fair rang (2.39 and 2.95 respectively, out of 5).

As a result of this analysis, PNNL recommends a more structured and flexible training curriculum with a blended approach; designed and implemented with audience specific learning objectives and delivery options for different users (operators, responders, technicians, managers, IT, etc.). This training would be piloted at RMS sites; with initial instructor led training as a required element of RMS installation, and a blended approach of instructor led and e-learning options for refresher training and reference materials, to facilitate sustainability of operations.

As for the software itself, the operational needs survey results do not indicate a need for major changes at this time. However, as per the training survey results, we now know that many of the users do not fully understand all of the RMS functions and capabilities. A more targeted analysis of more advanced users and system administrators may be required to fully analyze operational ease and efficiency of the system, and to gather more detailed input on user needs for potential future RMS development efforts.

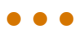

\section{Overview}

The RMS is a critical element of the GTRI protection strategy to mitigate threats to existing legal radioactive materials. Off-site monitoring of the RMS can be accomplished at locations including one or more of the following: local law enforcement, a commercial monitoring center, federal law enforcement, regulatory authority, or the military. The GTRI has adjusted the program strategy for RMS development and deployment, which has resulted in some significant changes to the current RMS development process. The current RMS development and deployment strategy has consisted of as-needed development and deployment activities. This approach will be replaced with a strategy to deploy a consistent and stable RMS configuration at all GTRI partner sites. The program is conducting a comprehensive RMS needs assessment to guide any further RMS development and training activities for the new strategy. This first phase of the assessment is focused on a training needs analysis and an operational analysis.

\section{Goals}

\section{Training Needs Analysis}

The primary purpose of the training needs analysis was to determine if the instruction being provided at the installation of an RMS unit was sufficient for proper operation and response to the system alerts and alarms. In addition, the survey team sought to find out if there were performance tasks that could be improved with specific training and what those areas might be.

\section{Operational Analysis}

The operational analysis was intended to gather information on the functionality of the software and seek input on how improvements might be made. User input is a critical part of the formal RMS 
development process. The resulting data and input will help guide focus for another portion of the development process - a more detailed user needs analysis.

\section{Objectives}

1. Design a survey based on the functional duties required to effectively monitor and interact with the RMS system interface.

2. Distribute the survey electronically to a wide array of end users as provided by GTRI Domestic Program Teams from PNNL and Sandia National Laboratories.

3. Retrieve survey data upon conclusion of the survey period.

4. Analyze data.

5. Present summary, conclusions, and recommendations in a written report.

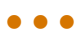

\section{Resources/Methodology}

Data analysis is the process of putting together qualitative and quantitative information to help derive answers to specific questions. Qualitative data must be analyzed for content and a determination made as to the intent of the information provided, whereas quantitative data can be provided in the form of numbers or numeric values generally on a scale from high to low, best to worst, etc. The raw data collected from analysis instruments is synthesized to form conclusions and recommendations to address the need for which the survey was first commissioned.

The first step in this training survey process was to review existing documentation on the RMS software to include the following YFH manuals:

1. GTRI Remote Monitoring System (RMS), Operator's Training Guide (V2.0 September 2010)

2. GTRI RMS Client Quick Start Training Guide (V2.10, February 2010)

A functional task list - duties performed by users through interaction with the RMS monitoring software - was developed from these materials and reviewed by members of the GTRI Domestic Materials Protection Program who are involved with site assessment and installations. The training needs survey needed to be based on task and function as opposed to job or position within the organization, as RMS users have a variety of other primary job duties. It was determined that a single training needs analysis survey would be designed and sent to the users of the RMS monitoring station computer and software. The list of users included:

- Radiation Safety Officer

- Dispatcher

- Site Operator

- Site Administrator

- Site Instructor/Trainer

- Site Responder

- Offsite Responder

- Law Enforcement
- Fire Service

- IT Specialist

- Security System Technician

- Site Security

- Security Manager/Director

- Assistant Security Manager/Director

- Other (to be specified in response) 
Once the list of tasks was finalized and approved, the survey was entered into the online survey tool ${ }^{2}$ for distribution. This data gathering platform was the most accessible for the majority of the proposed survey recipients, as they generally have computer access during their normal workday.

On March 19, 2012 the training survey invitation was distributed to 245 original recipients (Appendix G). The initial invitation (Appendix C) requested that each recipient "Please forward the survey links to all staff and vendors who monitor and interact with the Remote Monitoring System." The survey team was copied on at least one email where the initial recipient did indeed forward the survey to additional individuals. This was intended to broaden the pool of potential survey respondents. In addition, contact information for respondents not affiliated with PNNL RMS installations was requested from YFH who currently provides initial training with installation, and Sandia National Laboratories which also has work within the RMS network.

The survey process was unique in that it contained a "piggyback" survey on the operational satisfaction with the RMS. This was noted in the invitation and at the conclusion of the survey. A separate set of questions relating to the operability of the RMS system was developed and since many of the respondents would have been the same for this survey, it was distributed in a semi-combined fashion. As a result, 20 recipients responded to both the training and the operational assessments. The results of each will be addressed separately in the section below.

\section{Results}

\section{Training Needs Assessment}

92 responses were received for the training needs assessment for a return rate of $37.6 \%$ : a successful return rate ${ }^{3}$ indicative of targeting the proper audience and its desire to have feedback noted. It is significant to note that over $1 / 3$ of the respondents stated they had no training, or didn't know if they had been trained. At least half of the additional comments offered indicated that the training was "minimal" and that no formal training was provided.

\begin{tabular}{|l|c|c|}
\hline \multicolumn{2}{|l|}{ Question 14. Have you received any prior RMS software training? } \\
\hline Answer Options & $\begin{array}{c}\text { Response } \\
\text { Percent }\end{array}$ & $\begin{array}{c}\text { Response } \\
\text { Count }\end{array}$ \\
\hline Yes & $65.4 \%$ & 53 \\
\hline Not sure & $3.7 \%$ & 3 \\
\hline No & $30.9 \%$ & 25 \\
\hline Additional comments & & 18 \\
\hline
\end{tabular}

Respondents who stated they did receive prior training answered follow up questions on how well prepared they felt to perform various RMS functions. These questions resulted in a mixed response,

\footnotetext{
${ }^{2}$ The tool used to disseminate and gather data was Survey Monkey ${ }^{\circledR}$, an online survey design tool that provides 17 formats for asking questions, tracks respondents and non-respondents, sends out the survey and provides a link so that the survey can be forwarded to additional parties by the primary target audience.

${ }^{3}$ http://www.utexas.edu/academic/ctl/assessment/iar/teaching/gather/method/survey-Response.php Acceptable response rates vary by how the survey is administered: Mail: $50 \%$ adequate, $60 \%$ good, $70 \%$ very good, Email: $40 \%$ average, $50 \%$ good, $60 \%$ very good, Online: $30 \%$ average.
} 
however less than $36.2 \%$ reported being well-prepared to use the RMS software and less than $50 \%$ reported to understand what the alarms meant.

Question 16. Following the instruction you received, to what extent did you feel adequately
prepared to:
\begin{tabular}{|l|c|c|c|c|c|}
\hline Answer Options & $\begin{array}{c}\text { Not at all } \\
\text { prepared }\end{array}$ & $\begin{array}{c}\text { Somewhat } \\
\text { prepared }\end{array}$ & $\begin{array}{c}\text { Well } \\
\text { prepared }\end{array}$ & $\begin{array}{c}\text { Rating } \\
\text { Average }\end{array}$ & $\begin{array}{c}\text { Response } \\
\text { Count }\end{array}$ \\
\hline Use the RMS software? & $21.7 \%$ & $42.0 \%$ & $36.2 \%$ & 2.14 & 69 \\
\hline Monitor RMS alarms? & $14.5 \%$ & $37.7 \%$ & $47.8 \%$ & 2.33 & 69 \\
\hline Understand what the alarms meant? & $7.2 \%$ & $42.0 \%$ & $50.7 \%$ & 2.43 & 69 \\
\hline $\begin{array}{l}\text { Make proper notifications when } \\
\text { receiving alarms? }\end{array}$ & $10.1 \%$ & $34.8 \%$ & $55.1 \%$ & 2.45 & 69 \\
\hline Additional comments & & & & 11 \\
\hline
\end{tabular}

Comments included:

- No written manual. Prompts on screen are not adequate. More training - refresher is needed

- The instruction was conducted for a lot of people in a very busy office. There was no one-onone training

- More training should be provided at the time of installation of the RMS software

- Software is quite complicated. Would be good to have a hands-on demo with alarm scenarios. Our training was before the system was up and running so we never got to see actual alarms.

- Training was very brief, no way to know if it was complete

- Don't fully understand some of the alerts

- Lack of understanding on the part of our dispatchers

- The training took place a few months before system was in use. Did not remember specifics covered in training.

When queried in regard to what additional training they thought might be needed, $53.3 \%$ requested a software overview, $46.7 \%$ requested training on how to use the RMS software, and $65 \%$ requested annual refresher training in some form.

\begin{tabular}{|l|c|c|}
\hline \multicolumn{2}{|l|}{$\begin{array}{l}\text { Question 18. What other kinds of training would have helped you work with the RMS software? } \\
\text { Please select any/all that apply: }\end{array}$} & Response Percent \\
\hline Answer Options & Response Count \\
\hline $\begin{array}{l}\text { RMS Client Software Overview \{product } \\
\text { familiarization; software overview; status level } \\
\text { setup; setting alarm conditions; tabs (alarms, } \\
\text { general, sounds)\} }\end{array}$ & $53.3 \%$ & 32 \\
\hline $\begin{array}{l}\text { Using the RMS Client Software \{simple view } \\
\text { operation; live images; polled images; latest events; } \\
\text { radiation graph; student practice\} }\end{array}$ & $46.7 \%$ & 28 \\
\hline $\begin{array}{l}\text { RMS Alarms and Related Activities \{using the alarms } \\
\text { screen; alarm viewer (polled images, latest event, } \\
\text { radiation graph); acknowledge alarm actions; alarm } \\
\text { logs; information and warning messages\} }\end{array}$ & $41.7 \%$ & 25 \\
\hline One-time Refresher Training & & \\
\hline Quarterly Refresher/New Hire Training & $16.7 \%$ & 10 \\
\hline Annual Refresher Training & $21.7 \%$ & 39 \\
\hline
\end{tabular}


More significant data came from participant responses when evaluating self-proficiency for specific tasks, as respondents became aware of RMS functions that they were not previously known about. Overall for 26 separate tasks, respondents selected "I am Proficient at this Task" only $29.7 \%$ of the time and reported "I have minimal or no proficiency at this task" $20.9 \%$ of the time.

\begin{tabular}{|c|c|c|c|c|c|c|}
\hline $\begin{array}{l}\text { Question 21. If you use OR n } \\
\text { the following system tasks: }\end{array}$ & itor the & IS compu & please e & late your & rent sk & level with \\
\hline Answer Options & $\begin{array}{l}\text { I do not } \\
\text { perform } \\
\text { this task }\end{array}$ & $\begin{array}{c}\text { I have } \\
\text { minimal or } \\
\text { no } \\
\text { proficiency } \\
\text { at this task }\end{array}$ & $\begin{array}{l}\text { I am } \\
\text { proficient } \\
\text { at this } \\
\text { task }\end{array}$ & $\begin{array}{c}\text { I am } \\
\text { extremely } \\
\text { proficient } \\
\text { at this } \\
\text { task }\end{array}$ & $\begin{array}{l}\text { Rating } \\
\text { Average }\end{array}$ & $\begin{array}{l}\text { Response } \\
\text { Count }\end{array}$ \\
\hline $\begin{array}{l}\text { I can locate and open the } \\
\text { RMS software interface }\end{array}$ & $33.8 \%$ & $12.3 \%$ & $43.1 \%$ & $10.8 \%$ & 1.31 & 65 \\
\hline $\begin{array}{l}\text { I can locate and open the } \\
\text { RMS Viewer window }\end{array}$ & $33.8 \%$ & $9.2 \%$ & $44.6 \%$ & $12.3 \%$ & 1.35 & 65 \\
\hline $\begin{array}{l}\text { I can locate and open the } \\
\text { RMS Simple Viewer window }\end{array}$ & $35.4 \%$ & $9.2 \%$ & $43.1 \%$ & $12.3 \%$ & 1.32 & 65 \\
\hline $\begin{array}{l}\text { I can locate a specific RMS } \\
\text { device within the RMS } \\
\text { software interface }\end{array}$ & $34.8 \%$ & $15.2 \%$ & $39.4 \%$ & $10.6 \%$ & 1.26 & 66 \\
\hline $\begin{array}{l}\text { I understand the difference } \\
\text { between each status } \\
\text { indicator (good, marginal, } \\
\text { bad) }\end{array}$ & $30.8 \%$ & $18.5 \%$ & $40.0 \%$ & $10.8 \%$ & 1.31 & 65 \\
\hline $\begin{array}{l}\text { I can locate and view the } \\
\text { "Live View" }\end{array}$ & $27.7 \%$ & $6.2 \%$ & $52.3 \%$ & $13.8 \%$ & 1.52 & 65 \\
\hline $\begin{array}{l}\text { I can locate and view the } \\
\text { "Latest Events" }\end{array}$ & $28.8 \%$ & $15.2 \%$ & $43.9 \%$ & $12.1 \%$ & 1.39 & 66 \\
\hline $\begin{array}{l}\text { I can locate and view the } \\
\text { "Radiation Graph" }\end{array}$ & $25.8 \%$ & $16.7 \%$ & $45.5 \%$ & $12.1 \%$ & 1.44 & 66 \\
\hline $\begin{array}{l}\text { I can view individual graph } \\
\text { data point values }\end{array}$ & $34.4 \%$ & $20.3 \%$ & $32.8 \%$ & $12.5 \%$ & 1.23 & 64 \\
\hline $\begin{array}{l}\text { I can export the Radiation } \\
\text { Graph data }\end{array}$ & $51.6 \%$ & $29.7 \%$ & $12.5 \%$ & $6.3 \%$ & 0.73 & 64 \\
\hline $\begin{array}{l}\text { I can locate and view alarm } \\
\text { data }\end{array}$ & $29.2 \%$ & $15.4 \%$ & $44.6 \%$ & $10.8 \%$ & 1.37 & 65 \\
\hline $\begin{array}{l}\text { I can locate and view alerts } \\
\text { data }\end{array}$ & $29.7 \%$ & $20.3 \%$ & $39.1 \%$ & $10.9 \%$ & 1.31 & 64 \\
\hline $\begin{array}{l}\text { I can locate and view } \\
\text { radiation readings data }\end{array}$ & $29.2 \%$ & $16.9 \%$ & $41.5 \%$ & $12.3 \%$ & 1.37 & 65 \\
\hline $\begin{array}{l}\text { I can copy data rows to } \\
\text { paste into external } \\
\text { programs }\end{array}$ & $58.7 \%$ & $25.4 \%$ & $12.7 \%$ & $3.2 \%$ & 0.60 & 63 \\
\hline $\begin{array}{l}\text { I can enlarge RMS images or } \\
\text { image sections }\end{array}$ & $43.9 \%$ & $27.3 \%$ & $22.7 \%$ & $6.1 \%$ & 0.91 & 66 \\
\hline $\begin{array}{l}\text { I can check available disk } \\
\text { storage space for the RMS }\end{array}$ & $50.8 \%$ & $27.7 \%$ & $18.5 \%$ & $3.1 \%$ & 0.74 & 65 \\
\hline
\end{tabular}




\begin{tabular}{|l|l|l|l|l|l|l|}
\hline $\begin{array}{l}\text { software data on the } \\
\text { workstation }\end{array}$ & & & & & & \\
\hline $\begin{array}{l}\text { I can check the } \\
\text { communications status } \\
\text { between each RMS and the } \\
\text { workstation }\end{array}$ & $50.8 \%$ & $20.0 \%$ & $21.5 \%$ & $7.7 \%$ & 0.86 & 65 \\
\hline $\begin{array}{l}\text { I can check the status of } \\
\text { individual RMS components }\end{array}$ & $47.7 \%$ & $27.7 \%$ & $18.5 \%$ & $6.2 \%$ & 0.83 & 65 \\
\hline $\begin{array}{l}\text { I can enable data and alarm } \\
\text { filters for each RMS }\end{array}$ & $60.9 \%$ & $26.6 \%$ & $10.9 \%$ & $1.6 \%$ & 0.53 & 64 \\
\hline Additional comments & & & & & 10 \\
\hline
\end{tabular}

These responses clearly support the need for more comprehensive and structured training, especially since respondents could also have chosen "I do not perform this task." It also became evident that the survey respondents didn't realize how much they did not know, with comments such as: "Boy I really need some training after seeing this list", "So when is the next class?" and "Sounds like we should be invited to a course." Another telling comment was "The Laboratory's security department and two police departments probably have a total of 4 individuals out of over 100 that are truly proficient in the tasks described above. That is why there are a large number of mistakes made at the monitoring sites."

\section{Operational Needs Assessment}

The response rate on the operational need assessment was $17.1 \%$. The majority of the respondents to the survey indicated only having the RMS at their facility $1-2$ years (55.3\%). Their overall satisfaction with the system was in the good range (3.5 out of 5), however they rated "Ease of conducting maintenance procedures" and "Quality of helpdesk assistance" in the fair range with 2.39 and 2.95 respectively - therefore these areas may be considered as specific focus areas in any additional operational needs analysis activities.

Question 6. Indicate your overall satisfaction regarding the RMS software usability:

\begin{tabular}{|l|l|l|l|l|l|c|c|c|}
\hline Answer Options & Poor & Fair & Good & $\begin{array}{c}\text { Very } \\
\text { Good }\end{array}$ & Excellent & $\begin{array}{c}\text { Do Not } \\
\text { Recall/ } \\
\text { NA }\end{array}$ & $\begin{array}{c}\text { Rating } \\
\text { Average }\end{array}$ & $\begin{array}{c}\text { Response } \\
\text { Count }\end{array}$ \\
\hline $\begin{array}{l}\text { Ease of monitoring } \\
\text { alarms }\end{array}$ & $0.0 \%$ & $2.6 \%$ & $28.2 \%$ & $35.9 \%$ & $30.8 \%$ & $2.6 \%$ & 3.87 & 39 \\
\hline $\begin{array}{l}\text { Ease of navigating } \\
\text { software }\end{array}$ & $0.0 \%$ & $7.9 \%$ & $34.2 \%$ & $36.8 \%$ & $13.2 \%$ & $7.9 \%$ & 3.32 & 38 \\
\hline $\begin{array}{l}\text { Ease of assessing } \\
\text { alarm and alert } \\
\text { conditions }\end{array}$ & $2.6 \%$ & $0.0 \%$ & $28.9 \%$ & $34.2 \%$ & $28.9 \%$ & $5.3 \%$ & 3.71 & 38 \\
\hline $\begin{array}{l}\text { Accessibility of data } \\
\text { logs }\end{array}$ & $0.0 \%$ & $13.2 \%$ & $23.7 \%$ & $42.1 \%$ & $10.5 \%$ & $10.5 \%$ & 3.18 & 38 \\
\hline $\begin{array}{l}\text { Overall satisfaction } \\
\text { with the RMS } \\
\text { software }\end{array}$ & $0.0 \%$ & $7.7 \%$ & $28.2 \%$ & $38.5 \%$ & $20.5 \%$ & $5.1 \%$ & 3.56 & 39 \\
\hline
\end{tabular}




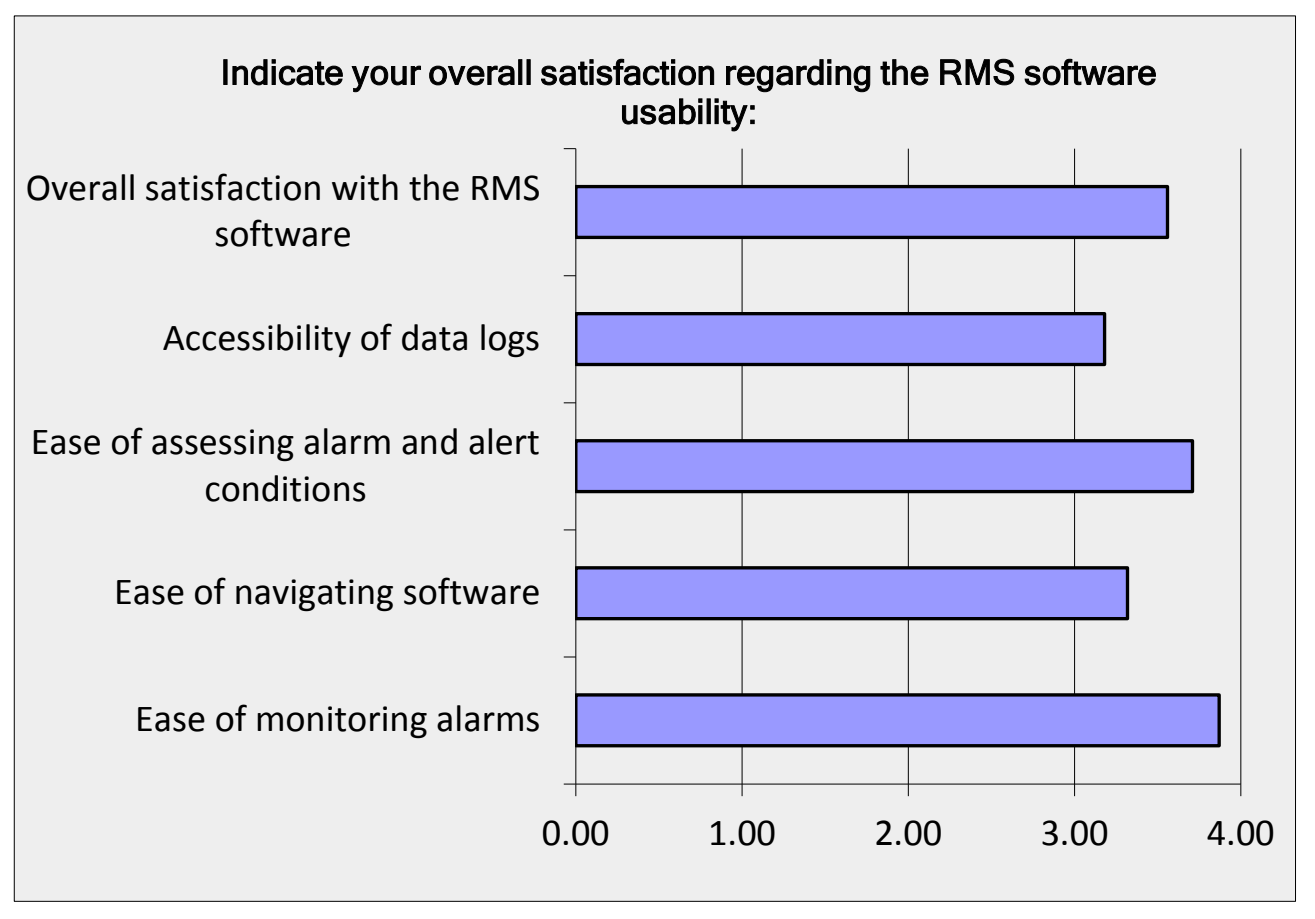

When asked about which RMS component or procedures needed improvement, almost $30 \%$ of the respondents answered that they were satisfied with the system, and another $27 \%$ stated they did not know the system well enough to make a determination regarding improvements.

It should be noted here that since nearly $32 \%$ of the respondents have had the system less than a year, there may not have been enough interaction with it for them to comfortably make an assessment of potential system improvements.

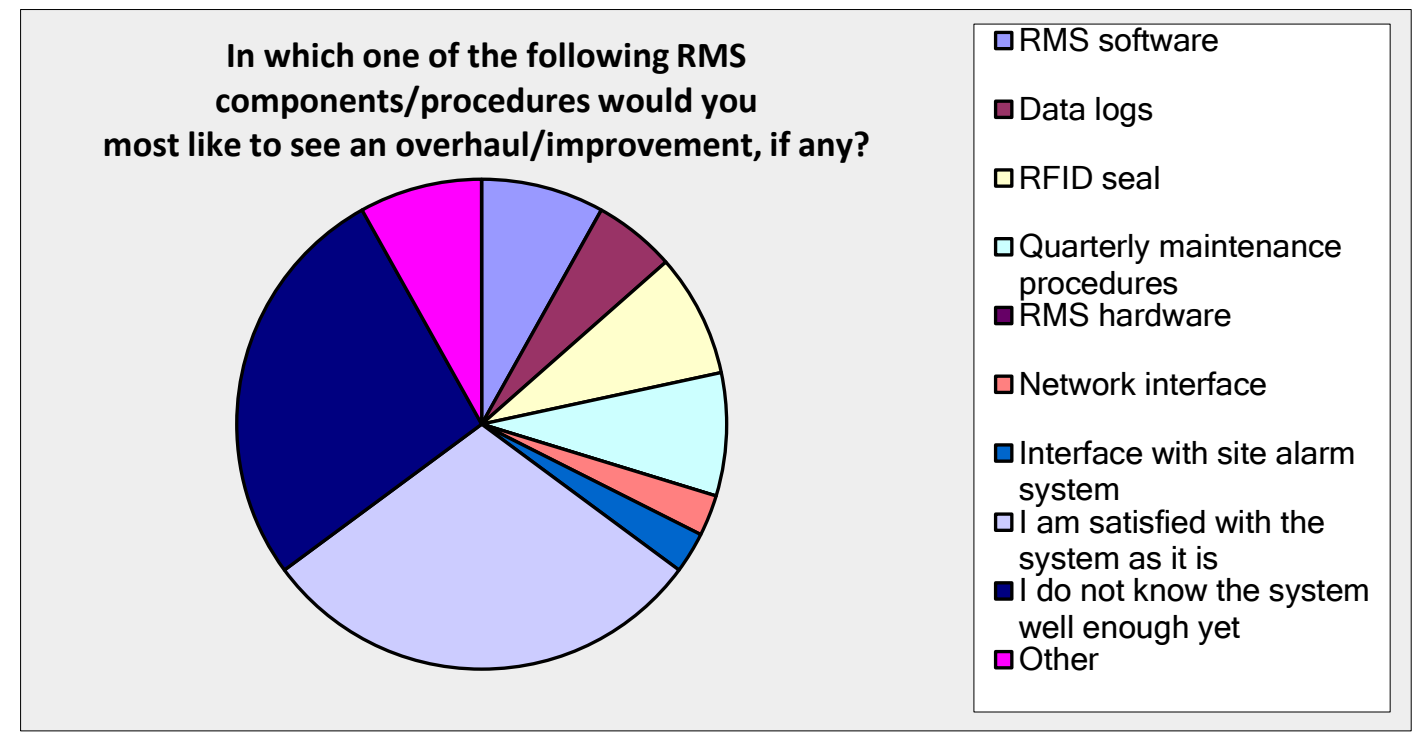




\section{Conclusions and Recommendations}

The information obtained from the training analysis sought to address three basic questions:

1. Is adequate training being provided to RMS sites at installation?

2. Are all users being trained?

3. What training is needed to help reduce the number of unnecessary or excessive external responses?

The answer to questions 1 and 2 is no; current training is not perceived as being adequate, nor does it seem to be reaching the appropriate audience in all cases. There is a need to develop structured, more formal, initial training, and annual or as needed refresher training. As for question 3 , the information obtained on individual tasks with which users were not even familiar can help determine the design and content of any revised training curriculum for specific RMS interface and operational duties.

The operational analysis showed that the RMS installations for most of the respondents were fairly recent $(55.3 \%$ reported less than two years and $31.9 \%$ were less than one year) and so they did not have a large amount of experience with the system. Still they reported overall satisfaction with the system, as indicated by results such as: good (28.2\%), very good (38.5\%), and excellent (20.5\%). Specific user recommendations for improvements in upcoming versions can be viewed in Appendix F.

To address the training concerns raised by the survey, the recommendation is two-fold. A formal instructor-led training course should be considered that can be delivered upon installation of the RMS unit by qualified GTRI personnel. Any training design should address the tasks and duties highlighted by this analysis. The training should make use of various training mediums and take a blended delivery approach for all users, with consideration to: thoroughness; ease of delivery; skill-based practice; and follow-up support as necessary. A training design document can outline this blended approach in more detail and will be generated using DOE-accepted instructional design principles upon approval of this recommendation.

Additionally the GTRI program may want to consider taking a broader view of training needs surrounding the entire system - users, installers, train-the-trainer for sites with training departments for continued onsite training efforts. 


\section{References}

Dillman, D. A. Mail and Internet Surveys: The Total Design Method (2nd Ed.). New York: Wiley, 2000.

Division of Instructional Innovation and Assessment, The University of Texas at Austin. "Guidelines for Maximizing Response Rates." Instructional Assessment Resources. 2007. <http://www.utexas.edu/academic/diia/assessment/iar/teaching/gather/method/surveyResponse.php>

Hamilton, M. B. "Online survey response rates and times: background and guidance for industry." Massachusetts: Tercent, Inc., 2003.

Punch, K. F. Survey Research: The Basics. London: Sage Publications Ltd, 2003.

Sheehan, K."E-mail Survey Response Rates: A Review." Journal of Computer-Mediated Communication, 2001: 6 .

SurveyMonkey ${ }^{\circledast}$. "Response Rates \& Surveying Techniques: Tips to Enhance Survey Respondent Participation." 2009. <http://s3.amazonaws.com/SurveyMonkeyFiles/Response_Rates.pdf > 


\section{Appendix A}

\section{Training Needs Assessment Survey Form \\ (Reprinted from SurveyMonkey ${ }^{\circledR}$ )}

\section{PAGE 1}

\section{$\underline{\text { Introduction }}$}

Welcome to the GTRI Remote Monitoring System (RMS) training needs assessment and thank you in advance for your time and feedback! As you know, the RMS is a real-time monitoring system to notify personnel of any attempted theft or sabotage of radiological or nuclear materials. The RMS monitoring software is an operator interface for the receipt and assessment of alarms via real time video, stored alarm images, alarm trigger data and radiation readings from the target area. You have been selected to participate in this brief survey because of your experiences with an RMS installation and the prior training you received as a part of that installation process. As a vital part of the global effort to combat nuclear and radiological terrorism, it is essential that you and your colleagues receive the training and tools you need to do your job - the results of this survey will help to make sure you have the tools you need.

Your candid and thoughtful feedback is anonymous and will be forwarded directly to our training developers. It will determine the content for new RMS software training and so is very important to the success of this effort. Please make sure you complete this survey - which should take you no more than 10 minutes - no later than $3 / 30 / 12$. At the end of this survey, there is additional opportunity to contact us directly, so please make sure to take the survey through to its conclusion. Thank you again for your input!

\section{PAGE 2}

\section{Background Information}

Q1

In what state or U.S. territory do you live?

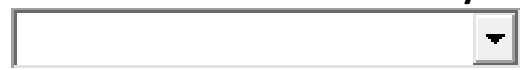

Q2

In what city do you live?

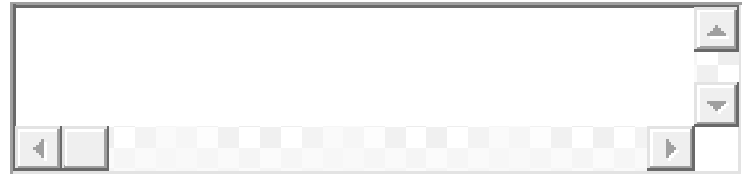

Q3

In which site or facility do you work?

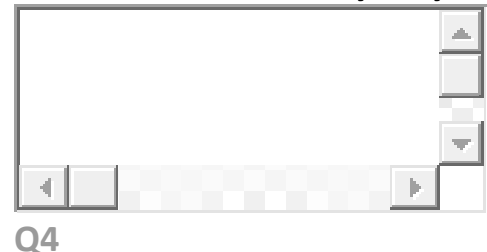

Position/primary role (choose all that apply): 


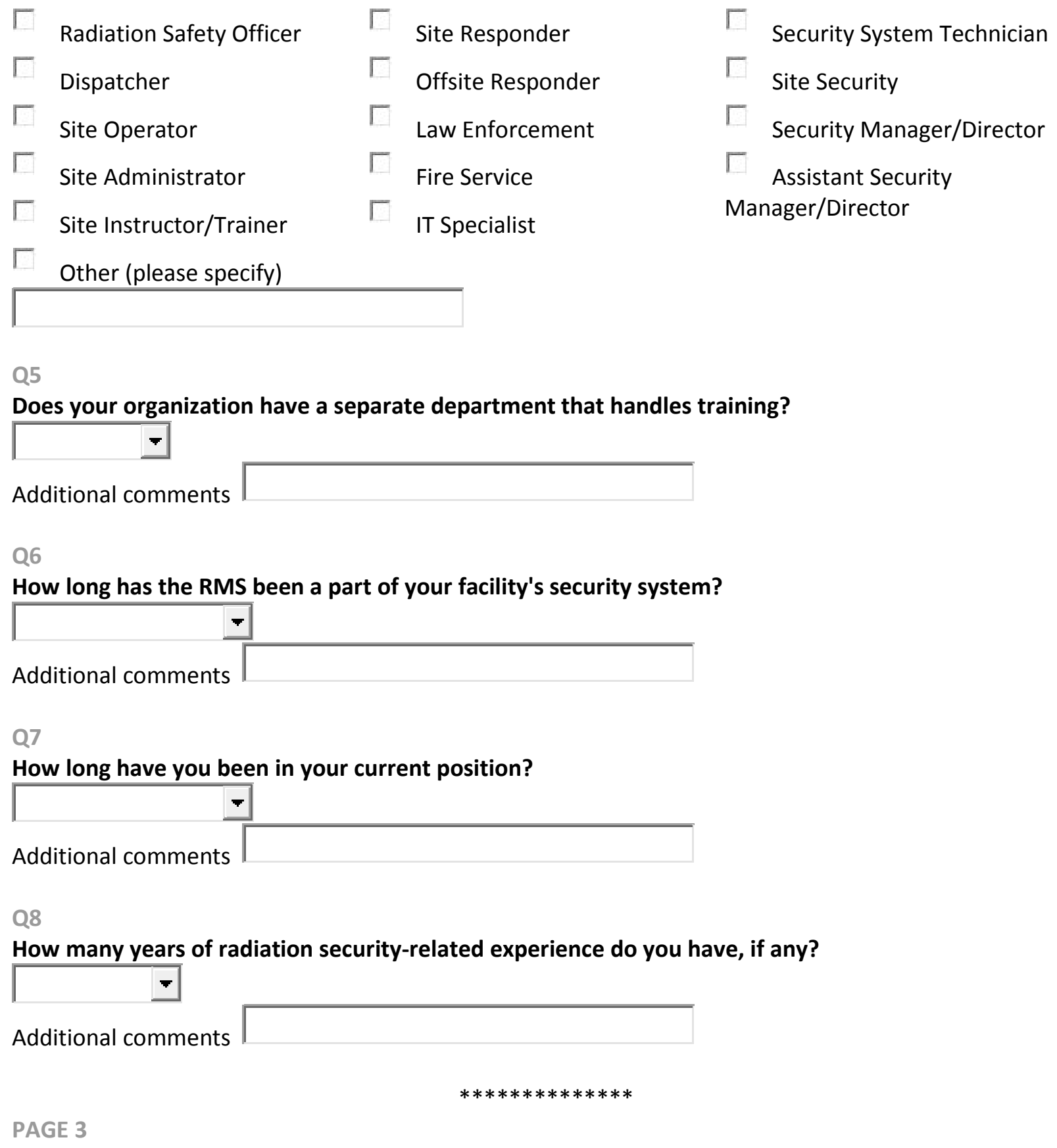

\section{Scheduling Preference}

Q9

Please select the most convenient day of the week for you to attend a training class:

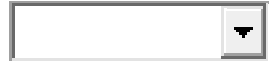

Additional comments

Q10

Please select the most convenient time during the day for you to attend a training class:

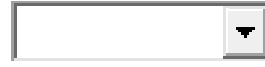

Additional comments 


\section{Job Duties}

\section{Q11}

Which of the following statements most accurately describes your responsibilities/interactions with the RMS software? Select all that apply

My job does not require any use or interaction with the RMS workstation.

$\Gamma$ I think the RMS software is installed on a workstation in my area, but I minimize it and do not really understand what it is for.

$\Gamma$

I monitor and review the RMS software data during my shift or on a daily/regular basis.

$\Gamma$ I oversee operator use of the RMS software data.

$\Gamma$ I review data as needed when alerted by the person monitoring the RMS.

$\Gamma$ Other (please specify)

\section{Q12}

How often do you review the RMS software data?

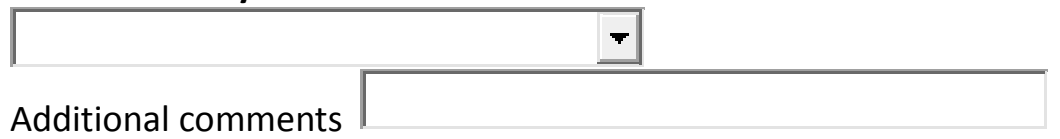

Q13

What percentage of your work day is spent monitoring the RMS software data?

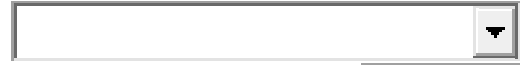

Additional comments

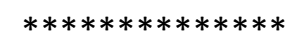

\section{PAGE 5}

\section{Prior RMS Software Training}

\section{Q14}

Have you received any prior RMS software training?

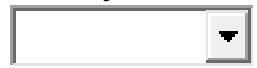

Additional comments 
Was the training too easy or too complex?

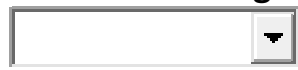

\section{Q16}

Following the instruction you received, to what extent did you feel adequately prepared to:

\begin{tabular}{|c|c|c|c|}
\hline & Not at all prepared & Somewhat prepared & Well prepared \\
\hline $\begin{array}{l}\text { Use the RMS } \\
\text { software? }\end{array}$ & Not at all prepared & Somewhat prepared & Well prepared \\
\hline $\begin{array}{l}\text { Monitor RMS } \\
\text { alarms? }\end{array}$ & Not at all prepared & Somewhat prepared & Well prepared \\
\hline $\begin{array}{l}\text { Understand what } \\
\text { the alarms meant? }\end{array}$ & Not at all prepared & Somewhat prepared & Well prepared \\
\hline $\begin{array}{l}\text { Make proper } \\
\text { notifications when } \\
\text { receiving alarms? }\end{array}$ & Not at all prepared & Somewhat prepared & Well prepared \\
\hline
\end{tabular}

If you did not feel prepared to use the RMS software, please explain why not:

\section{Q18}

What other kinds of training would have helped you work with the RMS software? Please select any/all that apply:

ГRMS Client Software Overview \{product familiarization; software overview; status level setup; setting alarm conditions; tabs (alarms, general, sounds)\}

$\Gamma$ Using the RMS Client Software \{simple view operation; live images; polled images; latest events; radiation graph; student practice $\}$

$\Gamma \quad$ RMS Alarms and Related Activities \{using the alarms screen; alarm viewer (polled images, latest event, radiation graph); acknowledge alarm actions; alarm logs; information and warning messages\}

$\Gamma \quad$ One-time Refresher Training

$\Gamma$ Quarterly Refresher/New Hire Training

$\Gamma$ Annual Refresher Training

Other (please specify)

Q19

Please rate the quality of the following elements of the training you received:

\begin{tabular}{|c|c|c|c|c|c|c|}
\hline 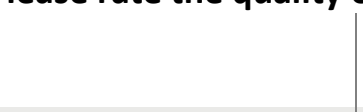 & Poor & Fair & Good & $\underline{\text { Very Good }}$ & Excellent & 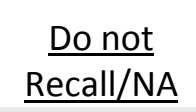 \\
\hline $\begin{array}{l}\text { Overall content of the } \\
\text { training }\end{array}$ & Poor & Fair & Good & $\begin{array}{l}\text { Very } \\
\text { Good }\end{array}$ & Excellent & $\begin{array}{l}\text { Do not } \\
\text { Recall/NA }\end{array}$ \\
\hline User $\xi$ & Poor & Fair & Good & Very & & Do not \\
\hline
\end{tabular}




\begin{tabular}{|c|c|c|c|c|c|c|}
\hline materials & & & & Good & Excellent & Recall/NA \\
\hline $\begin{array}{l}\text { Presentation of } \\
\text { material by Instructor }\end{array}$ & Poor & Fair & Good & $\begin{array}{l}\text { Very } \\
\text { Good }\end{array}$ & Excellent & $\begin{array}{l}\text { Do not } \\
\text { Recall/NA }\end{array}$ \\
\hline $\begin{array}{l}\text { Participant/Group } \\
\text { activities or actual } \\
\text { practice during } \\
\text { training with the RMS } \\
\text { software }\end{array}$ & Poor & Fair & Good & $\begin{array}{l}\text { Very } \\
\text { Good }\end{array}$ & Excellent & $\begin{array}{l}\text { Do not } \\
\text { Recall/NA }\end{array}$ \\
\hline $\begin{array}{l}\text { Facilitation of } \\
\text { activities by } \\
\text { Instructor }\end{array}$ & Poor & Fair & Good & $\begin{array}{l}\text { Very } \\
\text { Good }\end{array}$ & Excellent & $\begin{array}{l}\text { Do not } \\
\text { Recall/NA }\end{array}$ \\
\hline
\end{tabular}

Additional comments

Q20

Please share any other comments you have that would help us improve the next RMS software training.

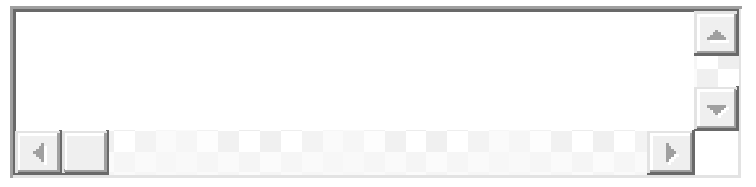

\section{PAGE 6}

\section{Current RMS Software Use and Software Response Skills}

\section{Q21}

If you use OR monitor the RMS computer, please evaluate your current skill level with the following system tasks:

\begin{tabular}{|c|c|c|c|c|}
\hline & $\frac{\text { I do not perform }}{\text { this task }}$ & $\frac{\text { I have minimal or }}{\frac{\text { no proficiency at }}{\text { this task }}}$ & $\frac{\text { I am proficient at }}{\underline{\text { this task }}}$ & $\frac{\frac{\text { I am extremely }}{\text { proficient at this }}}{\underline{\text { task }}}$ \\
\hline $\begin{array}{l}\text { I can locate and } \\
\text { open the RMS } \\
\text { software interface }\end{array}$ & $\begin{array}{l}\text { I do not perform } \\
\text { this task }\end{array}$ & $\begin{array}{l}\text { I have minimal } \\
\text { or no proficiency at } \\
\text { this task }\end{array}$ & $\begin{array}{c}\text { I am proficient at } \\
\text { this task }\end{array}$ & $\begin{array}{l}\text { I am extremely } \\
\text { proficient at this } \\
\text { task }\end{array}$ \\
\hline $\begin{array}{l}\text { I can locate and } \\
\text { open the RMS } \\
\text { Viewer window }\end{array}$ & $\begin{array}{l}\text { I do not perform } \\
\text { this task }\end{array}$ & $\begin{array}{l}\text { I have minimal } \\
\text { or no proficiency at } \\
\text { this task }\end{array}$ & $\begin{array}{c}\text { I am proficient at } \\
\text { this task }\end{array}$ & $\begin{array}{c}\text { I am extremely } \\
\text { proficient at this } \\
\text { task }\end{array}$ \\
\hline $\begin{array}{l}\text { I can locate and } \\
\text { open the RMS } \\
\text { Simple Viewer } \\
\text { window }\end{array}$ & $\begin{array}{l}\text { I do not perform } \\
\text { this task }\end{array}$ & $\begin{array}{l}\text { I have minimal } \\
\text { or no proficiency at } \\
\text { this task }\end{array}$ & $\begin{array}{c}\text { I am proficient at } \\
\text { this task }\end{array}$ & $\begin{array}{c}\text { I am extremely } \\
\text { proficient at this } \\
\text { task }\end{array}$ \\
\hline $\begin{array}{l}\text { I can locate a } \\
\text { specific RMS device }\end{array}$ & $\begin{array}{l}\text { I do not perform } \\
\text { this task }\end{array}$ & $\begin{array}{l}\text { I have minimal } \\
\text { or no proficiency at }\end{array}$ & $\begin{array}{c}\text { I am proficient at } \\
\text { this task }\end{array}$ & $\begin{array}{l}\text { I am extremely } \\
\text { proficient at this }\end{array}$ \\
\hline
\end{tabular}




\begin{tabular}{|c|c|c|c|c|}
\hline $\begin{array}{l}\text { within the RMS } \\
\text { software interface }\end{array}$ & & this task & & task \\
\hline $\begin{array}{l}\text { I understand the } \\
\text { difference between } \\
\text { each status indicator } \\
\text { (good, marginal, } \\
\text { bad) }\end{array}$ & $\begin{array}{l}\text { I do not perform } \\
\text { this task }\end{array}$ & $\begin{array}{c}\text { I have minimal } \\
\text { or no proficiency at } \\
\text { this task }\end{array}$ & $\begin{array}{c}\text { I am proficient at } \\
\text { this task }\end{array}$ & $\begin{array}{c}\text { I am extremely } \\
\text { proficient at this } \\
\text { task }\end{array}$ \\
\hline $\begin{array}{l}\text { I can locate and } \\
\text { view the "Live View" }\end{array}$ & $\begin{array}{l}\text { I do not perform } \\
\text { this task }\end{array}$ & $\begin{array}{l}\text { I have minimal } \\
\text { or no proficiency at } \\
\text { this task }\end{array}$ & $\begin{array}{c}\text { I am proficient at } \\
\text { this task }\end{array}$ & $\begin{array}{c}\text { I am extremely } \\
\text { proficient at this } \\
\text { task }\end{array}$ \\
\hline $\begin{array}{l}\text { I can locate and } \\
\text { view the "Latest } \\
\text { Events" }\end{array}$ & $\begin{array}{l}\text { I do not perform } \\
\text { this task }\end{array}$ & $\begin{array}{l}\text { I have minimal } \\
\text { or no proficiency at } \\
\text { this task }\end{array}$ & $\begin{array}{c}\text { I am proficient at } \\
\text { this task }\end{array}$ & $\begin{array}{c}\text { I am extremely } \\
\text { proficient at this } \\
\text { task }\end{array}$ \\
\hline $\begin{array}{l}\text { I can locate and } \\
\text { view the "Radiation } \\
\text { Graph" }\end{array}$ & $\begin{array}{l}\text { I do not perform } \\
\text { this task }\end{array}$ & $\begin{array}{l}\text { I have minimal } \\
\text { or no proficiency at } \\
\text { this task }\end{array}$ & $\begin{array}{c}\text { I am proficient at } \\
\text { this task }\end{array}$ & $\begin{array}{l}\text { I am extremely } \\
\text { proficient at this } \\
\text { task }\end{array}$ \\
\hline $\begin{array}{l}\text { I can view individual } \\
\text { graph data point } \\
\text { values }\end{array}$ & $\begin{array}{l}\text { I do not perform } \\
\text { this task }\end{array}$ & $\begin{array}{c}\text { I have minimal } \\
\text { or no proficiency at } \\
\text { this task }\end{array}$ & $\begin{array}{c}\text { I am proficient at } \\
\text { this task }\end{array}$ & $\begin{array}{c}\text { I am extremely } \\
\text { proficient at this } \\
\text { task }\end{array}$ \\
\hline $\begin{array}{l}\text { I can export the } \\
\text { Radiation Graph } \\
\text { data }\end{array}$ & $\begin{array}{l}\text { I do not perform } \\
\text { this task }\end{array}$ & $\begin{array}{l}\text { I have minimal } \\
\text { or no proficiency at } \\
\text { this task }\end{array}$ & $\begin{array}{c}\text { I am proficient at } \\
\text { this task }\end{array}$ & $\begin{array}{c}\text { I am extremely } \\
\text { proficient at this } \\
\text { task }\end{array}$ \\
\hline $\begin{array}{l}\text { I can locate and } \\
\text { view alarm data }\end{array}$ & $\begin{array}{l}\text { I do not perform } \\
\text { this task }\end{array}$ & $\begin{array}{l}\text { I have minimal } \\
\text { or no proficiency at } \\
\text { this task }\end{array}$ & $\begin{array}{c}\text { I am proficient at } \\
\text { this task }\end{array}$ & $\begin{array}{c}\text { I am extremely } \\
\text { proficient at this } \\
\text { task }\end{array}$ \\
\hline $\begin{array}{l}\text { I can locate and } \\
\text { view alerts data }\end{array}$ & $\begin{array}{l}\text { I do not perform } \\
\text { this task }\end{array}$ & $\begin{array}{c}\text { I have minimal } \\
\text { or no proficiency at } \\
\text { this task }\end{array}$ & $\begin{array}{c}\text { I am proficient at } \\
\text { this task }\end{array}$ & $\begin{array}{c}\text { I am extremely } \\
\text { proficient at this } \\
\text { task }\end{array}$ \\
\hline $\begin{array}{l}\text { I can locate and } \\
\text { view radiation } \\
\text { readings data }\end{array}$ & $\begin{array}{l}\text { I do not perform } \\
\text { this task }\end{array}$ & $\begin{array}{l}\text { I have minimal } \\
\text { or no proficiency at } \\
\text { this task }\end{array}$ & $\begin{array}{c}\text { I am proficient at } \\
\text { this task }\end{array}$ & $\begin{array}{l}\text { I am extremely } \\
\text { proficient at this } \\
\text { task }\end{array}$ \\
\hline $\begin{array}{l}\text { I can copy data rows } \\
\text { to paste into } \\
\text { external programs }\end{array}$ & $\begin{array}{c}\text { I do not perform } \\
\text { this task }\end{array}$ & $\begin{array}{l}\text { I have minimal } \\
\text { or no proficiency at } \\
\text { this task }\end{array}$ & $\begin{array}{c}\text { I am proficient at } \\
\text { this task }\end{array}$ & $\begin{array}{l}\text { I am extremely } \\
\text { proficient at this } \\
\text { task }\end{array}$ \\
\hline $\begin{array}{l}\text { I can enlarge RMS } \\
\text { images or image }\end{array}$ & $\begin{array}{l}\text { I do not perform } \\
\text { this task }\end{array}$ & $\begin{array}{l}\text { I have minimal } \\
\text { or no proficiency at }\end{array}$ & $\begin{array}{c}\text { I am proficient at } \\
\text { this task }\end{array}$ & $\begin{array}{l}\text { I am extremely } \\
\text { proficient at this }\end{array}$ \\
\hline
\end{tabular}




\begin{tabular}{|c|c|c|c|c|}
\hline sections & & this task & & task \\
\hline $\begin{array}{l}\text { I can check available } \\
\text { disk storage space } \\
\text { for the RMS } \\
\text { software data on } \\
\text { the workstation }\end{array}$ & $\begin{array}{l}\text { I do not perform } \\
\text { this task }\end{array}$ & $\begin{array}{c}\text { I have minimal } \\
\text { or no proficiency at } \\
\text { this task }\end{array}$ & $\begin{array}{c}\text { I am proficient at } \\
\text { this task }\end{array}$ & $\begin{array}{c}\text { I am extremely } \\
\text { proficient at this } \\
\text { task }\end{array}$ \\
\hline $\begin{array}{l}\text { I can check the } \\
\text { communications } \\
\text { status between each } \\
\text { RMS and the } \\
\text { workstation }\end{array}$ & $\begin{array}{l}\text { I do not perform } \\
\text { this task }\end{array}$ & $\begin{array}{c}\text { I have minimal } \\
\text { or no proficiency at } \\
\text { this task }\end{array}$ & $\begin{array}{c}\text { I am proficient at } \\
\text { this task }\end{array}$ & $\begin{array}{c}\text { I am extremely } \\
\text { proficient at this } \\
\text { task }\end{array}$ \\
\hline $\begin{array}{l}\text { I can check the } \\
\text { status of individual } \\
\text { RMS components }\end{array}$ & $\begin{array}{l}\text { I do not perform } \\
\text { this task }\end{array}$ & $\begin{array}{l}\text { I have minimal } \\
\text { or no proficiency at } \\
\text { this task }\end{array}$ & $\begin{array}{l}\text { I am proficient at } \\
\text { this task }\end{array}$ & $\begin{array}{c}\text { I am extremely } \\
\text { proficient at this } \\
\text { task }\end{array}$ \\
\hline $\begin{array}{l}\text { I can enable data } \\
\text { and alarm filters for } \\
\text { each RMS }\end{array}$ & $\begin{array}{l}\text { I do not perform } \\
\text { this task }\end{array}$ & $\begin{array}{c}\text { I have minimal } \\
\text { or no proficiency at } \\
\text { this task }\end{array}$ & $\begin{array}{l}\text { I am proficient at } \\
\text { this task }\end{array}$ & $\begin{array}{c}\text { I am extremely } \\
\text { proficient at this } \\
\text { task }\end{array}$ \\
\hline
\end{tabular}

\section{Q22}

Software Response Tasks

\begin{tabular}{|c|c|c|c|c|}
\hline & $\frac{\text { d do not perform this }}{\text { task }}$ & $\mid \frac{\text { proficiency at this }}{\underline{\text { task }}}$ & $\frac{\text { I am proficient at }}{\text { this task }}$ & $\frac{\frac{\text { I am extremely }}{\text { proficient at this }}}{\text { task }}$ \\
\hline $\begin{array}{l}\text { I know how to } \\
\text { contact the System } \\
\text { Administrator }\end{array}$ & $\begin{array}{c}\text { I do not } \\
\text { perform this task }\end{array}$ & $\begin{array}{c}\text { I have minimal } \\
\text { or no proficiency at } \\
\text { this task }\end{array}$ & $\begin{array}{l}\text { I am proficient } \\
\text { at this task }\end{array}$ & $\begin{array}{l}\text { I am extremely } \\
\text { proficient at this } \\
\text { task }\end{array}$ \\
\hline $\begin{array}{l}\text { I know when to } \\
\text { contact the System } \\
\text { Administrator }\end{array}$ & $\begin{array}{c}\text { I do not } \\
\text { perform this task }\end{array}$ & $\begin{array}{l}\text { I have minimal } \\
\text { or no proficiency at } \\
\text { this task }\end{array}$ & $\begin{array}{l}\text { I am proficient } \\
\text { at this task }\end{array}$ & $\begin{array}{l}\text { I am extremely } \\
\text { proficient at this } \\
\text { task }\end{array}$ \\
\hline $\begin{array}{l}\text { I know how to } \\
\text { contact the GTRI } \\
\text { RMS Help Desk }\end{array}$ & $\begin{array}{l}\text { I do not } \\
\text { perform this task }\end{array}$ & $\begin{array}{l}\text { I have minimal } \\
\text { or no proficiency at } \\
\text { this task }\end{array}$ & $\begin{array}{l}\text { I am proficient } \\
\text { at this task }\end{array}$ & $\begin{array}{l}\text { I am extremely } \\
\text { proficient at this } \\
\text { task }\end{array}$ \\
\hline $\begin{array}{l}\text { I know when to } \\
\text { contact the GTRI } \\
\text { RMS Help Desk }\end{array}$ & $\begin{array}{l}\text { I do not } \\
\text { perform this task }\end{array}$ & $\begin{array}{l}\text { I have minimal } \\
\text { or no proficiency at } \\
\text { this task }\end{array}$ & $\begin{array}{l}\text { I am proficient } \\
\text { at this task }\end{array}$ & $\begin{array}{l}\text { I am extremely } \\
\text { proficient at this } \\
\text { task }\end{array}$ \\
\hline $\begin{array}{l}\text { I recognize the } \\
\text { audio alarm signal } \\
\text { from the } \\
\text { monitoring } \\
\text { workstation }\end{array}$ & $\begin{array}{l}\text { I do not } \\
\text { perform this task }\end{array}$ & $\begin{array}{l}\text { I have minimal } \\
\text { or no proficiency at } \\
\text { this task }\end{array}$ & $\begin{array}{l}\text { I am proficient } \\
\text { at this task }\end{array}$ & $\begin{array}{l}\text { I am extremely } \\
\text { proficient at this } \\
\text { task }\end{array}$ \\
\hline
\end{tabular}




\begin{tabular}{|c|c|c|c|c|}
\hline $\begin{array}{l}\text { I know the } \\
\text { appropriate } \\
\text { response for a } \\
\text { "Seal Left" alert }\end{array}$ & $\begin{array}{c}\text { I do not } \\
\text { perform this task }\end{array}$ & $\begin{array}{l}\text { I have minimal } \\
\text { or no proficiency at } \\
\text { this task }\end{array}$ & $\begin{array}{l}\text { I am proficient } \\
\text { at this task }\end{array}$ & $\begin{array}{l}\text { I am extremely } \\
\text { proficient at this } \\
\text { task }\end{array}$ \\
\hline $\begin{array}{l}\text { I know the } \\
\text { appropriate } \\
\text { response for a } \\
\text { "Radnet Trigger" } \\
\text { alarm }\end{array}$ & $\begin{array}{l}\text { I do not } \\
\text { perform this task }\end{array}$ & $\begin{array}{l}\text { I have minimal } \\
\text { or no proficiency at } \\
\text { this task }\end{array}$ & $\begin{array}{l}\text { I am proficient } \\
\text { at this task }\end{array}$ & $\begin{array}{l}\text { I am extremely } \\
\text { proficient at this } \\
\text { task }\end{array}$ \\
\hline $\begin{array}{l}\text { I know the } \\
\text { appropriate } \\
\text { response for a } \\
\text { "RFID Seal" alarm }\end{array}$ & $\begin{array}{c}\text { I do not } \\
\text { perform this task }\end{array}$ & $\begin{array}{l}\text { I have minimal } \\
\text { or no proficiency at } \\
\text { this task }\end{array}$ & $\begin{array}{l}\text { I am proficient } \\
\text { at this task }\end{array}$ & $\begin{array}{l}\text { I am extremely } \\
\text { proficient at this } \\
\text { task }\end{array}$ \\
\hline $\begin{array}{l}\text { I know the } \\
\text { appropriate } \\
\text { response for a } \\
\text { "Housing" alarm }\end{array}$ & $\begin{array}{l}\text { I do not } \\
\text { perform this task }\end{array}$ & $\begin{array}{l}\text { I have minimal } \\
\text { or no proficiency at } \\
\text { this task }\end{array}$ & $\begin{array}{l}\text { I am proficient } \\
\text { at this task }\end{array}$ & $\begin{array}{l}\text { I am extremely } \\
\text { proficient at this } \\
\text { task }\end{array}$ \\
\hline $\begin{array}{l}\text { I know the } \\
\text { appropriate } \\
\text { response for an } \\
\text { "Intrusion" alarm }\end{array}$ & $\begin{array}{c}\text { I do not } \\
\text { perform this task }\end{array}$ & $\begin{array}{l}\text { I have minimal } \\
\text { or no proficiency at } \\
\text { this task }\end{array}$ & $\begin{array}{l}\text { I am proficient } \\
\text { at this task }\end{array}$ & $\begin{array}{l}\text { I am extremely } \\
\text { proficient at this } \\
\text { task }\end{array}$ \\
\hline $\begin{array}{l}\text { I know the } \\
\text { appropriate } \\
\text { response for a } \\
\text { "Duress" alarm }\end{array}$ & $\begin{array}{c}\text { I do not } \\
\text { perform this task }\end{array}$ & $\begin{array}{l}\text { I have minimal } \\
\text { or no proficiency at } \\
\text { this task }\end{array}$ & $\begin{array}{l}\text { I am proficient } \\
\text { at this task }\end{array}$ & $\begin{array}{l}\text { I am extremely } \\
\text { proficient at this } \\
\text { task }\end{array}$ \\
\hline $\begin{array}{l}\text { I know the } \\
\text { appropriate } \\
\text { response for a } \\
\text { "Trigger" alarm }\end{array}$ & $\begin{array}{c}\text { I do not } \\
\text { perform this task }\end{array}$ & $\begin{array}{l}\text { I have minimal } \\
\text { or no proficiency at } \\
\text { this task }\end{array}$ & $\begin{array}{l}\text { I am proficient } \\
\text { at this task }\end{array}$ & $\begin{array}{l}\text { I am extremely } \\
\text { proficient at this } \\
\text { task }\end{array}$ \\
\hline dditional commer & & & & \\
\hline
\end{tabular}

PAGE 7

\section{Contact Information (Optional)}

Q23

Name

Q24

Title

Q25

Phone Number 


\section{Additional Feedback Opportunities}

Do you perform alarm maintenance or information technology functions within the RMS? If so, provide additional feedback on operational satisfaction by pasting the following link into a new browser: https://www.surveymonkey.com/s/RMS Operational Satisfaction Assessment Survey Please feel free to forward this survey to other alarm maintenance or information technology individuals in your organization. If you have any questions, concerns, or additional feedback about the RMS please contact any of the below GTRI individuals.

Sorcha Fox, Instructional Designer

Global Threat Reduction Program

Pacific Northwest National Laboratory

509-372-6342

sorcha.fox@pnnl.gov

Debra Day, CPT, PMP, Senior Security Operations Specialist

Global Threat Reduction Program

Pacific Northwest National Laboratory

509-371-6255

debra.day@pnnl.gov

Mike Henry, Remote Monitoring System Coordinator

Office of Global Threat Reduction

955 L'Enfant Plaza, SW

Washington, DC 20585

202-586-3755

michael.henry@nnsa.doe.gov 


\title{
Appendix B
}

\author{
Operational Assessment Survey Form \\ (Reprinted from SurveyMonkey ${ }^{\circledR}$ )
}

\section{PAGE 1}

\section{$\underline{\text { Introduction }}$}

Welcome to the GTRI Remote Monitoring System (RMS) operational satisfaction assessment and thank you in advance for your time and feedback! The GTRI program is currently conducting a review of the entire RMS, including: software, hardware, infrastructure, and integration with site security systems, and you have been selected to participate in this survey because of your experience with an RMS installation. Your candid, thoughtful feedback is anonymous and will help identify areas of concern and opportunities for improvement to the RMS. The survey should take no more than 10 minutes and needs to be completed by $3 / 30 / 12$. If you previously completed our training needs assessment, you will recognize a few of the demographic questions. We need to ask for those responses again as the two surveys are not connected except by the active link. At the end of this survey, there is an opportunity to contact us directly, so please make sure to take the survey through to its conclusion. Thank you again for your input.

\section{PAGE 2}

\section{Background Information}

Q1

In what state or U.S. territory do you live?

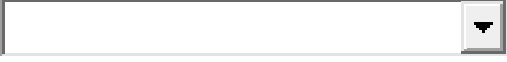

Q2

In what city do you live?

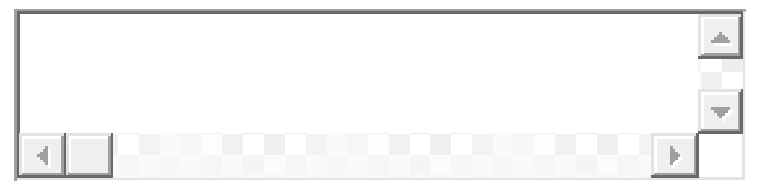

Q3

In which site or facility do you work?

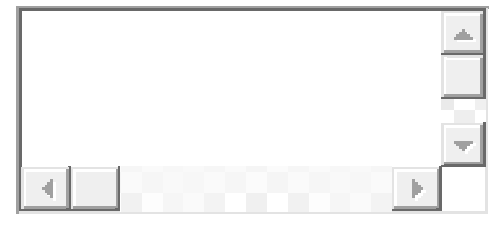

Q4

Position/primary role (choose all that apply): 


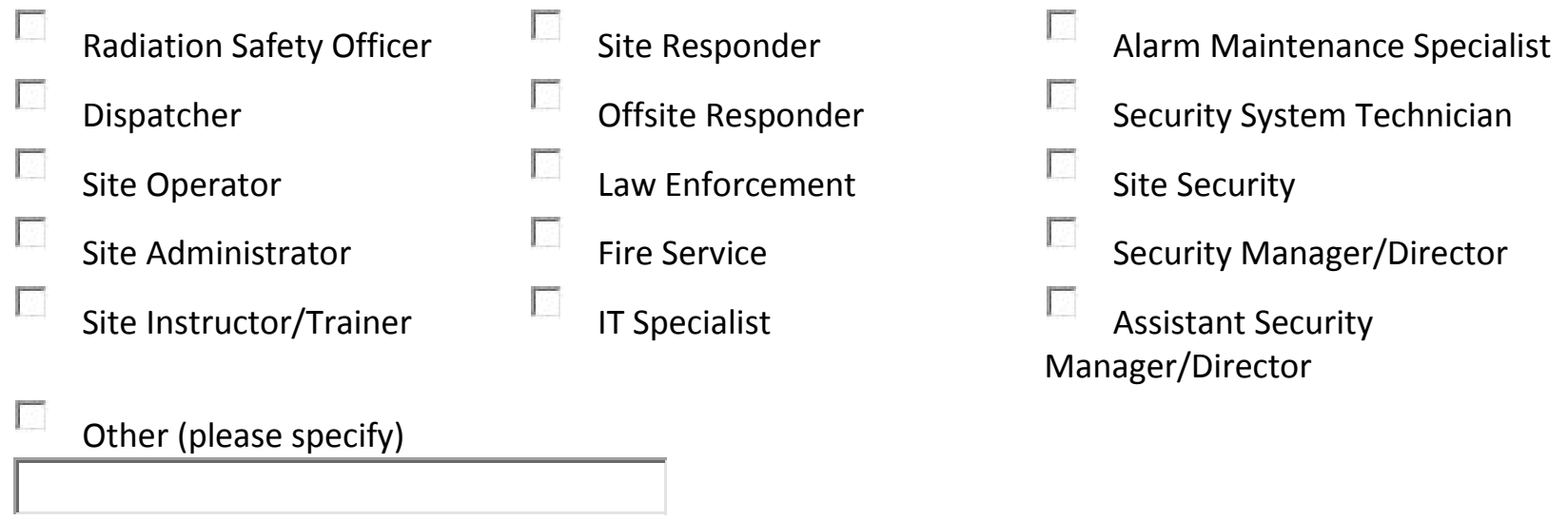

Q5

How long has the RMS been a part of your facility's security system?

Additional comments

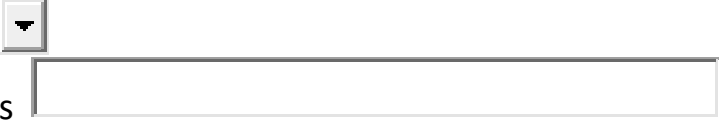

\section{PAGE 3}

\section{$\underline{\text { RMS Satisfaction }}$}

Q6

Indicate your overall satisfaction regarding the RMS software usability:

\begin{tabular}{|c|c|c|c|c|c|c|}
\hline & $\underline{\text { Poor }}$ & $\underline{\text { Fair }}$ & Good & $\underline{\text { Very Good }}$ & Excellent & $\underline{\text { Do Not }}$ \\
\hline $\begin{array}{l}\text { Ease of } \\
\text { monitoring } \\
\text { alarms }\end{array}$ & Poor & Fair & Good & $\begin{array}{l}\text { Very } \\
\text { Good }\end{array}$ & Excellent & $\begin{array}{l}\text { Do Not } \\
\text { Recall/NA }\end{array}$ \\
\hline $\begin{array}{l}\text { Ease of } \\
\text { navigating } \\
\text { software }\end{array}$ & Poor & Fair & Good & $\begin{array}{l}\text { Very } \\
\text { Good }\end{array}$ & Excellent & $\begin{array}{l}\text { Do Not } \\
\text { Recall/NA }\end{array}$ \\
\hline $\begin{array}{l}\text { Ease of } \\
\text { assessing alarm } \\
\text { and alert } \\
\text { conditions }\end{array}$ & Poor & Fair & Good & $\begin{array}{l}\text { Very } \\
\text { Good }\end{array}$ & Excellent & $\begin{array}{l}\text { Do Not } \\
\text { Recall/NA }\end{array}$ \\
\hline $\begin{array}{l}\text { Accessibility of } \\
\text { data logs }\end{array}$ & Poor & Fair & Good & $\begin{array}{l}\text { Very } \\
\text { Good }\end{array}$ & Excellent & $\begin{array}{l}\text { Do Not } \\
\text { Recall/NA }\end{array}$ \\
\hline $\begin{array}{l}\text { Overall } \\
\text { satisfaction } \\
\text { with the RMS } \\
\text { software }\end{array}$ & Poor & Fair & Good & $\begin{array}{l}\text { Very } \\
\text { Good }\end{array}$ & - Excellent & $\begin{array}{l}\text { Do Not } \\
\text { Recall/NA }\end{array}$ \\
\hline
\end{tabular}

Q7

Please provide feedback on best practices and/or potential areas for system improvement of the RMS software: 


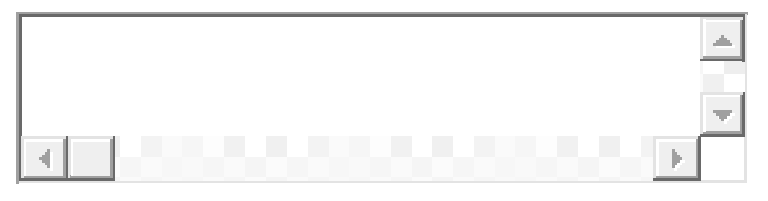

Q8

Please indicate your overall satisfaction regarding the RMS hardware and infrastructure usability:

\begin{tabular}{|c|c|c|c|c|c|c|}
\hline - & Poor & $\underline{\text { Fair }}$ & Good & Very Good & Excellent & $\underline{\text { Do Not }}$ \\
\hline $\begin{array}{l}\text { Acceptability of } \\
\text { false alarm rate } \\
\text { (frequency of } \\
\text { incidents) }\end{array}$ & Poor & Fair & Good & $\begin{array}{l}\text { Very } \\
\text { Good }\end{array}$ & Excellent & $\begin{array}{l}\text { Do Not } \\
\text { Recall/NA }\end{array}$ \\
\hline $\begin{array}{l}\text { Acceptability of } \\
\text { nuisance alarm } \\
\text { rate (frequency } \\
\text { of incidents) }\end{array}$ & Poor & Fair & Good & $\begin{array}{l}\text { Very } \\
\text { Good }\end{array}$ & Excellent & $\begin{array}{l}\text { Do Not } \\
\text { Recall/NA }\end{array}$ \\
\hline $\begin{array}{l}\text { Quality of } \\
\text { video images }\end{array}$ & Poor & Fair & Good & $\begin{array}{l}\text { Very } \\
\text { Good }\end{array}$ & Excellent & $\begin{array}{l}\text { Do Not } \\
\text { Recall/NA }\end{array}$ \\
\hline $\begin{array}{l}\text { Integration } \\
\text { with house } \\
\text { alarm system }\end{array}$ & Poor & Fair & Good & $\begin{array}{l}\text { Very } \\
\text { Good }\end{array}$ & Excellent & $\begin{array}{l}\text { Do Not } \\
\text { Recall/NA }\end{array}$ \\
\hline $\begin{array}{l}\text { Reliability of } \\
\text { RFID seal }\end{array}$ & Poor & Fair & Good & $\begin{array}{l}\text { Very } \\
\text { Good }\end{array}$ & Excellent & $\begin{array}{l}\text { Do Not } \\
\text { Recall/NA }\end{array}$ \\
\hline $\begin{array}{l}\text { Reliability of } \\
\text { network } \\
\text { interface }\end{array}$ & Poor & Fair & Good & $\begin{array}{l}\text { Very } \\
\text { Good }\end{array}$ & Excellent & $\begin{array}{l}\text { Do Not } \\
\text { Recall/NA }\end{array}$ \\
\hline $\begin{array}{l}\text { Reliability of } \\
\text { RMS hardware }\end{array}$ & Poor & Fair & Good & $\begin{array}{l}\text { Very } \\
\text { Good }\end{array}$ & Excellent & $\begin{array}{l}\text { Do Not } \\
\text { Recall/NA }\end{array}$ \\
\hline $\begin{array}{l}\text { Ease of } \\
\text { conducting } \\
\text { maintenance } \\
\text { procedures }\end{array}$ & Poor & Fair & Good & $\begin{array}{l}\text { Very } \\
\text { Good }\end{array}$ & Excellent & $\begin{array}{l}\text { Do Not } \\
\text { Recall/NA }\end{array}$ \\
\hline $\begin{array}{l}\text { Quality of help } \\
\text { desk assistance }\end{array}$ & Poor & Fair & Good & $\begin{array}{l}\text { Very } \\
\text { Good }\end{array}$ & Excellent & $\begin{array}{l}\text { Do Not } \\
\text { Recall/NA }\end{array}$ \\
\hline $\begin{array}{l}\text { Overall stability } \\
\text { of the system }\end{array}$ & Poor & Fair & Good & $\begin{array}{l}\text { Very } \\
\text { Good }\end{array}$ & Excellent & $\begin{array}{l}\text { Do Not } \\
\text { Recall/NA }\end{array}$ \\
\hline $\begin{array}{l}\text { Overall } \\
\text { satisfaction } \\
\text { with RMS? }\end{array}$ & Poor & Fair & Good & $\begin{array}{l}\text { Very } \\
\text { Good }\end{array}$ & Excellent & $\begin{array}{l}\text { Do Not } \\
\text { Recall/NA }\end{array}$ \\
\hline
\end{tabular}


Q9

In which one of the following RMS components/procedures would you most like to see an overhaul/improvement, if any?

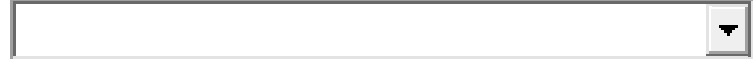

If other, please specify:

\section{Q10}

Please provide feedback on best practices and/or potential areas for system improvement of the complete RMS:

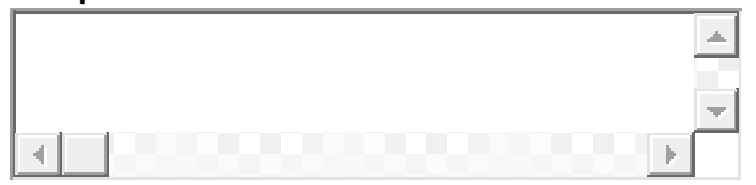

\section{Contact Information (Optional)}

\section{Q11}

Name

Q

Q12

Title

Q13

Phone Number

Q14

E-mail Address

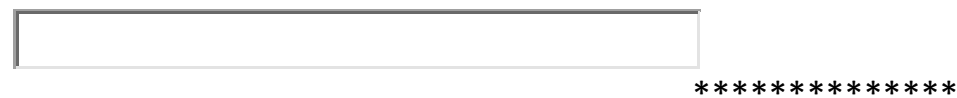

\section{Additional Feedback Opportunities}

Thank you for your input! If you have any questions, concerns, or additional feedback about the RMS please contact:

Mike Henry

Remote Monitoring System Coordinator

Office of Global Threat Reduction

955 L'Enfant Plaza, SW

Washington, DC 20585

202-586-3755

michael.henry@nnsa.doe.gov 


\section{Appendix C}

\section{Survey Invitation}

Attention RMS User,

You recently received an email invitation to participate in two surveys about training and operations of the Remote Monitoring System installed at your facility. We wanted to follow up and let you know just how important this survey is in deciding what future training will be made available for system users and administrators, and that we also need your input to make improvements in the operational aspects of the system. Just in case your system flagged the email as a possible problem, we are providing the links to both surveys again below:

Training Assessment Survey:

https://www.surveymonkey.com/s.aspx?sm=Kej 2f6tpl4CUgV 2f69lnm60Q 3d 3d

Operational Assessment Survey:

https://www.surveymonkey.com/s/RMS Operational Satisfaction Assessment Survey

These will take less than 10 minutes of your time and will provide us with critical information for making the RMS better; please be sure to take both of them.

You were selected because you are a primary RMS site contact however we need input from others who interact with the system as well. Please forward the survey links to all staff and vendors who monitor and interact with the Remote Monitoring System. Those individuals may include:

$\begin{array}{ll}\text { Radiation Safety Officer } & \text { Fire Service } \\ \text { Dispatcher } & \text { IT Specialist } \\ \text { Site Operator } & \text { Security System Technician } \\ \text { Site Administrator } & \text { Site Security } \\ \text { Site Instructor/Trainer } & \text { Security Manager/Director } \\ \text { Site Responder } & \text { Assistant Security Manager/Director } \\ \text { Offsite Responder } & \text { Security Vendor or Other Provider }\end{array}$

We understand your time is valuable and appreciate your willingness to help in this effort. Both surveys will close on 3/30/12. If you have any questions, please feel free to contact your PNNL POC or any of the survey team directly:

Debra Day, PNNL Senior Security Specialist, 509-371-6255, debra.day@pnnl.gov

Sorcha Fox, PNNL Instructional Designer, 509-372-6342, sorcha.fox@pnnl.gov

Mike Henry, Remote Monitoring System Coordinator, 202-586-3755, michael.henry@nnsa.doe.gov

Sincerely,

$<$ name $>$

RMS Survey Team

Return to Table of Contents 


\section{Appendix D}

\section{Survey Reminder}

Attention RMS Users,

The RMS training assessment and operational assessment surveys will end tomorrow - so if you haven't had a chance to complete them yet, please do so, and if you already have, thank you! These will take less than 10 minutes of your time and will provide us with critical information for making the RMS better; please be sure to take both of them. Here are the links to both surveys:

Training Assessment Survey:

https://www.surveymonkey.com/s/RMS Training Needs Assessment Survey

Operational Assessment Survey:

https://www.surveymonkey.com/s/RMS Operational Satisfaction Assessment Survey

Again, please forward the survey links to all staff and vendors who monitor and interact with the Remote Monitoring System. Those individuals may include:

- Radiation Safety Officer

- Dispatcher

- Site Operator

- Site Administrator

- Site Instructor/Trainer

- Site Responder

- Offsite Responder

- Fire Service
- IT Specialist

- Security System Technician

- Site Security

- Security Manager/Director

- Assistant Security Manager/Director

- Security Vendor or other provider

- Law Enforcement

We understand your time is valuable and appreciate your willingness to help in this effort. If you have any questions, please feel free to contact me or any of the survey team directly:

- Debra Day, PNNL Senior Security Specialist, 509-371-6255, debra.day@pnnl.gov

- Sorcha Fox, PNNL Instructional Designer, 509-372-6342, sorcha.fox@pnnl.gov

- Mike Henry, Remote Monitoring System Coordinator, 202-586-3755, michael.henry@nnsa.doe.gov

Sincerely,

$<$ name $>$

RMS Survey Team

$\underline{\text { Return to Table of Contents }}$ 
Training Needs Assessment:

Data Tables and Graphs

\section{Question 1. In what state or U.S. territory do you live?}

\begin{tabular}{|l|c|c|}
\hline Answer Options & Response Percent & Response Count \\
\hline Connecticut & $3.4 \%$ & 3 \\
\hline District of Columbia & $1.1 \%$ & 1 \\
\hline Illinois & $10.3 \%$ & 9 \\
\hline Indiana & $1.1 \%$ & 1 \\
\hline Maryland & $12.6 \%$ & 11 \\
\hline Massachusetts & $8.0 \%$ & 7 \\
\hline Mississippi & $1.1 \%$ & 1 \\
\hline Missouri & $8.0 \%$ & 7 \\
\hline Montana & $3.4 \%$ & 3 \\
\hline New Jersey & $2.3 \%$ & 2 \\
\hline New York & $25.3 \%$ & 22 \\
\hline Ohio & $2.3 \%$ & 7 \\
\hline Pennsylvania & $8.0 \%$ & 1 \\
\hline Puerto Rico & $1.1 \%$ & 3 \\
\hline Rhode Island & $3.4 \%$ & 1 \\
\hline Texas & $1.1 \%$ & 5 \\
\hline Washington & $5.7 \%$ & 1 \\
\hline Wisconsin & $1.1 \%$ & 2 \\
\hline
\end{tabular}

\section{Question 2. In what city do you live? \\ Response/Counts}

\begin{tabular}{|l|l|l|}
\hline Baltimore & Highlands & Pleasant Prairie \\
\hline Bethesda & Houston & Plymouth \\
\hline Blue Bell & Kansas City (5) & Prefer Not to Answer \\
\hline Boston (2) & Kensington & Providence (2) \\
\hline Boyd's & Kirkland & Raymore \\
\hline Chelmsford & Lee's Summit & Seattle (4) \\
\hline Chicago (7) & Lowell & Southaven \\
\hline Cincinnati (2) & Maywood & State College (2) \\
\hline Cold Spring Harbor (2) & Millville & Rochester (2) \\
\hline College Park (3) & Needham & Rockville (3) \\
\hline Cortland Manor & New Britain & Schenectady \\
\hline Cranston & New Hyde Park & Suburbs \\
\hline Demotte & New York (12) & Tacoma \\
\hline Gaithersburg & North Haven & Valhalla \\
\hline Hamilton (2) & Northborough & Washington, DC \\
\hline & Philadelphia (4) & Yonkers \\
\hline
\end{tabular}




\begin{tabular}{|l|l|}
\hline \multicolumn{2}{|l|}{ Question 3. In which site or facility do you work? } \\
Response/Count & Penn-Jersey Region \\
\hline Albany Medical Center & Prefer Not to Answer for Security Reasons \\
\hline Brown University (4) & Puget Sound Blood Center (2) \\
\hline Cambridge & Rocky Mountain Laboratories (3) \\
\hline Casco Security & Rush University Medical Center, Chicago, IL \\
\hline Children's Hospital Boston & Shady Grove Adventist Hospital (2) \\
\hline Children's Mercy Hospital (6) & Temple University \\
\hline Cold Spring Harbor Laboratory (2) & The Methodist Hospital Research Institute \\
\hline Columbia University (4) & George Washington Medical Center \\
\hline Drexel University, Philadelphia, Pennsylvania & The Rockefeller University \\
\hline Evanston \& Chicago & Tufts Medical Center \\
\hline Fox Chase Cancer Center & UIC \\
\hline Harvard University PD & Uniformed Services University of the Health \\
\hline & Sciences (USUHS) \\
\hline Hospital Lifeblood & University of Chicago (4) \\
\hline Medical Center & University of Cincinnati (2) \\
\hline Medstar Washington Hospital Center & University of Connecticut Health Center (2) \\
\hline Merck and Co. & University of Illinois \\
\hline MIT Montefiore Medical Center & University of Maryland (5) \\
\hline Mount Sinai Medical Center (4) & University of Massachusetts Lowell \\
\hline National Institutes of Health & University of Pennsylvania \\
\hline New York Presbyterian Hospital & University of Washington \\
\hline NIST Northwest Hospital & \\
\hline NYU Langone Medical Center/ Bellevue Hospital \\
(2) & Vaccinex \\
\hline PD U of I Chicago & VACT \\
\hline Penn State University (2) & Washington Alarm, Inc. Seattle, WA \\
\hline & Westchester Medical Center \\
\hline & \\
\hline
\end{tabular}

\section{Question 4. Position/primary role (choose all that apply):}

\begin{tabular}{|l|c|c|}
\hline Answer Options & Response Percent & Response Count \\
\hline Radiation Safety Officer & $43.7 \%$ & 38 \\
\hline Dispatcher & $8.0 \%$ & 7 \\
\hline Site Operator & $5.7 \%$ & 5 \\
\hline Site Administrator & $12.6 \%$ & 11 \\
\hline Site Instructor/Trainer & $11.5 \%$ & 10 \\
\hline Site Responder & $9.2 \%$ & 8 \\
\hline Offsite Responder & $1.1 \%$ & 1 \\
\hline Law Enforcement & $9.2 \%$ & 8 \\
\hline Fire Service & $1.1 \%$ & 1 \\
\hline IT Specialist & $2.3 \%$ & 2 \\
\hline Security System Technician & $5.7 \%$ & 5 \\
\hline
\end{tabular}




\begin{tabular}{|l|c|c|}
\hline Site Security & $11.5 \%$ & 10 \\
\hline Security Manager/Director & $11.5 \%$ & 10 \\
\hline Assistant Security Manager/Director & $6.9 \%$ & 6 \\
\hline Other (please specify) & $10.3 \%$ & 9 \\
\hline
\end{tabular}

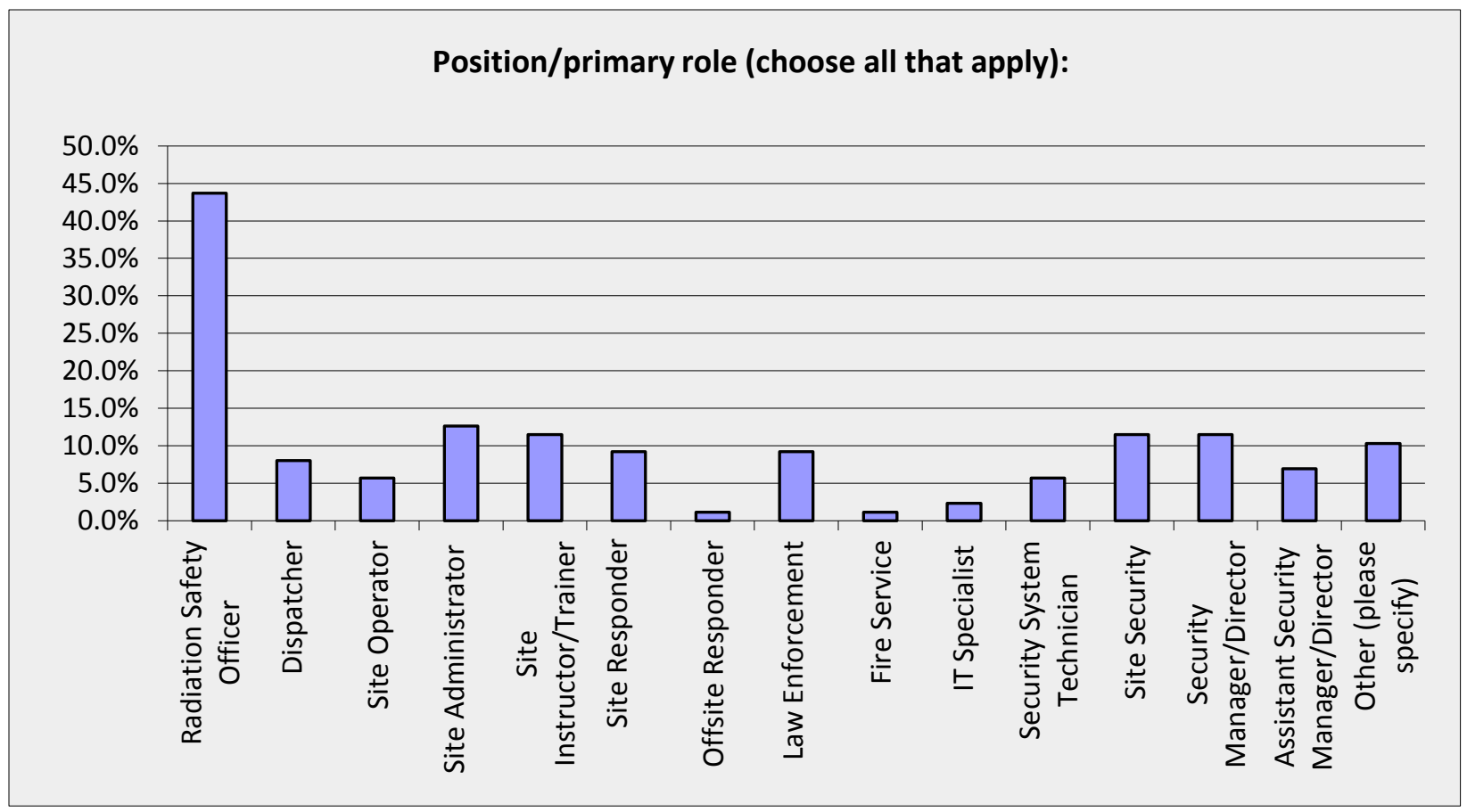

\begin{tabular}{|l|c|c|}
\hline \multicolumn{2}{|l|}{ Question 5. Does your organization have a separate department that handles training? } \\
\hline Answer Options & Response Percent & Response Count \\
\hline Yes & $24.1 \%$ & 21 \\
\hline Not sure & $4.6 \%$ & 4 \\
\hline No & $71.3 \%$ & 62 \\
\hline Additional comments & & 12 \\
\hline
\end{tabular}

\section{Comments:}

- I am the Training Academy Director

- Radiation Safety Dept. handles radioactive materials training

- Radiation Safety Office, Department of Environmental Safety

- Radiation Safety Department

- We give training only about Radiation and Lasers

- Depends on the training

- Radiation Safety Office conducts radiation related training

- What type of training? Many departments do their own training

- I offer training often times

- Routine training done in house. Specialized is contracted.

- All Safety training is performed by us 


\begin{tabular}{|l|c|c|}
\hline Question 6. How long has the RMS been a part of your facility's security system? \\
\hline Answer Options & Response Percent & Response Count \\
\hline Less than 1 year & $45.3 \%$ & 39 \\
\hline 1-2 years & $45.3 \%$ & 39 \\
\hline 3-4 years & $7.0 \%$ & 6 \\
\hline 5+ years & $2.3 \%$ & 2 \\
\hline Additional comments & & 7 \\
\hline
\end{tabular}

\section{Comments:}

- 5 ARC site up and running on RMS, 5 more being installed

- 2-3 years

- Unsure

- We have had them for years but they have just become important

- RMS has been live less than a year

- Installed Sep 2009

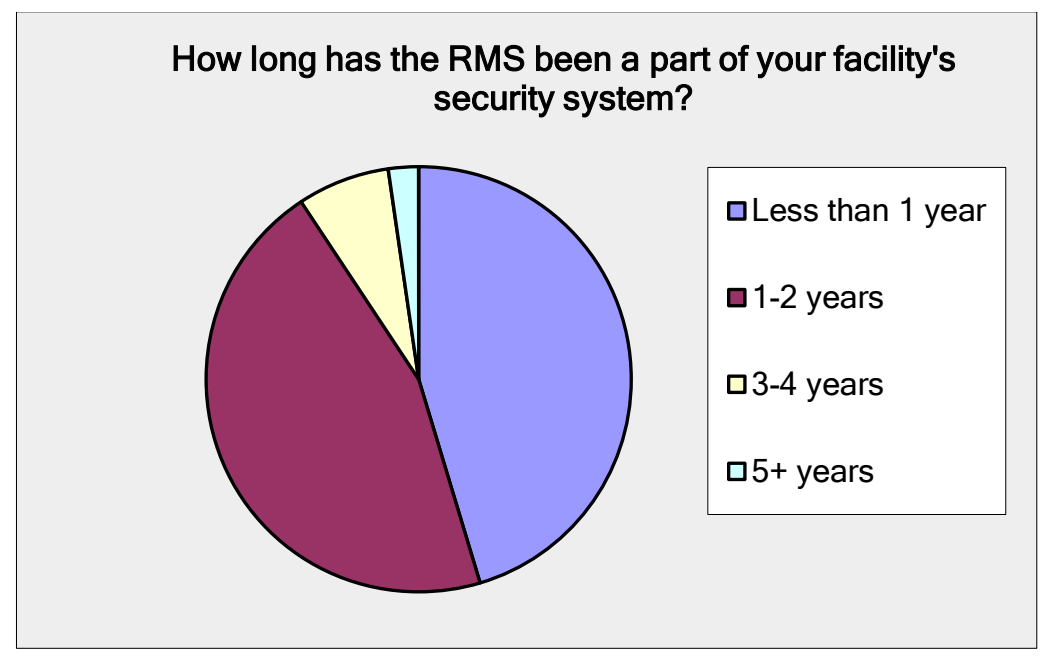

\section{Question 7. How long have you been in your current position?}

\begin{tabular}{|l|c|c|}
\hline Answer Options & Response Percent & Response Count \\
\hline Less than 1 year & $2.3 \%$ & 2 \\
\hline 1-2 years & $10.3 \%$ & 9 \\
\hline 3-4 years & $16.1 \%$ & 14 \\
\hline 5+ years & $71.3 \%$ & 62 \\
\hline
\end{tabular}




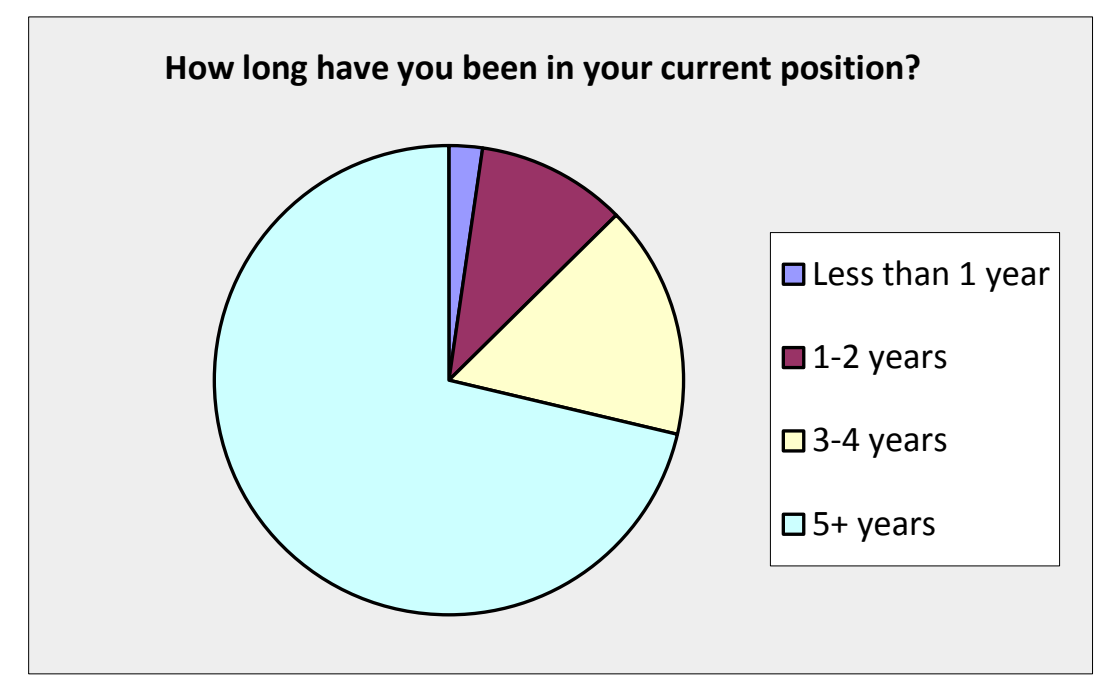

\begin{tabular}{|l|c|c|}
\hline Question 8. How many years of radiation security-related experience do you have, if any? \\
\hline Answer Options & Response Percent & Response Count \\
\hline None & $12.6 \%$ & 11 \\
\hline $0-1$ years & $6.9 \%$ & 6 \\
\hline $1-2$ years & $6.9 \%$ & 6 \\
\hline 3-4 years & $16.1 \%$ & 14 \\
\hline $5+$ years & $57.5 \%$ & 50 \\
\hline
\end{tabular}

\section{Comments:}

- OJT since the NRC increased controls went into effect

- No training before we went to Tennessee

- All my radiation security-related experience is via our GTRI on-the-job deployment.

- 20 years

- This became more focused when the IC order went into effect

- Just what I have received in this position.

- Been doing this since 1980

- More than 10 years 
How many years of radiation security-related experience do you have, if any?

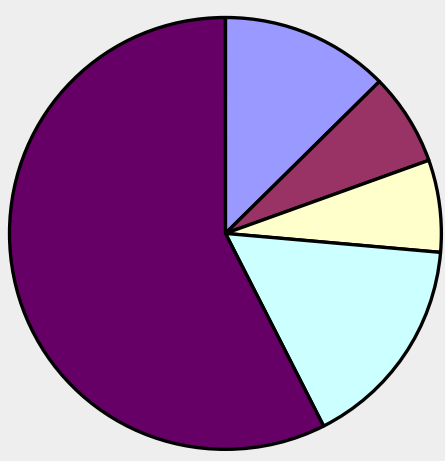

$\square$ None

$\square 0-1$ years

$\square 1-2$ years

$\square$ 3-4 years

$\square+$ years

\begin{tabular}{|l|c|c|}
\hline Question 9. Please select the most convenient day of the week for you to attend a training class: \\
\hline Answer Options & Response Percent & Response Count \\
\hline Monday & $17.7 \%$ & 14 \\
\hline Tuesday & $24.1 \%$ & 19 \\
\hline Wednesday & $29.1 \%$ & 23 \\
\hline Thursday & $11.4 \%$ & 9 \\
\hline Friday & $16.5 \%$ & 13 \\
\hline Saturday & $1.3 \%$ & 1 \\
\hline Sunday & $0.0 \%$ & 0 \\
\hline Additional comments & & 22 \\
\hline
\end{tabular}

\section{Comments:}

- Tuesday-Thursday

- Sunday through Saturday

- Generally Monday and Friday, but this term I will be teaching those days, so Tues. or Thurs.

- Varies according to work schedule

- My day off

- Tuesday thru Thursday

- It depends on the week

- Since I have to make sure the office is covered there is really no convenient time.

- But any day is generally fine.

- Any weekday will work

- online training is the best option for me

- Need training for RADEYE system

- Retiring in three months

- It would depend on where it is

- Any weekday is really ok - if onsite training

- Anytime but the weekends

- Most days are fine with advanced notice

- Any work day is really about the same as any other. 
- Any weekday is fine

- If travel is involved, any day mid-week is best

- Any day is OK

- Flexible depending on location e.g., travel

Please select the most convenient day of the week for you to attend a training class:

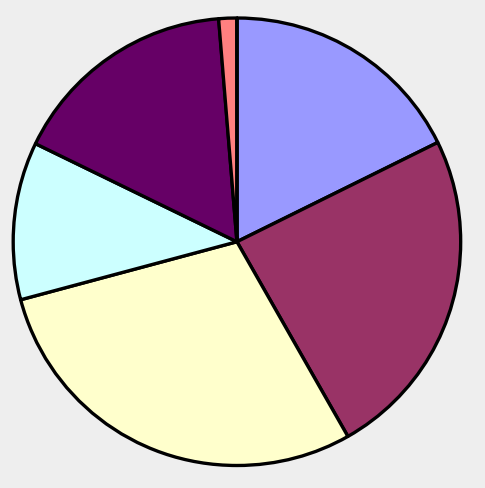

口Monday

口Tuesday

口Wednesday

口Thursday

口Friday

口Saturday

口unday

\begin{tabular}{|l|c|c|}
\hline $\begin{array}{l}\text { Question 10. Please select the most convenient time during the day for you to attend a training } \\
\text { class: }\end{array}$ & Response Percent & Response Count \\
\hline Answer Options & $67.5 \%$ & 54 \\
\hline Morning & $32.5 \%$ & 26 \\
\hline Afternoon & $0.0 \%$ & 0 \\
\hline Evening & & 14 \\
\hline Additional comments & & \\
\hline
\end{tabular}

\section{Comments:}

- Any time is the day is good for me

- All Three

- Afternoons or evenings

- It depends

- Please see above

- But any time is generally fine

- Or afternoon

- Retiring. No training needed.

- It would depend on where it is

- Anytime but the evening

- Anytime during normal working day, with advanced notice

- Morning or afternoon are really about the same.

- 8AM - 5 PM

- Flexible 


\begin{tabular}{|l|c|c|}
\hline $\begin{array}{l}\text { Question 11. Which of the following statements most accurately describes your } \\
\text { responsibilities/interactions with the RMS software? Select all that apply. }\end{array}$ & Response Count \\
\hline Answer Options & Response Percent & 14 \\
\hline $\begin{array}{l}\text { My job does not require any use or interaction with } \\
\text { the RMS workstation. }\end{array}$ & $17.1 \%$ & 1 \\
\hline $\begin{array}{l}\text { I think the RMS software is installed on a } \\
\text { workstation in my area, but I minimize it and do not } \\
\text { really understand what it is for. }\end{array}$ & $1.2 \%$ & 23 \\
\hline $\begin{array}{l}\text { I monitor and review the RMS software data during } \\
\text { my shift or on a daily/regular basis. }\end{array}$ & $28.0 \%$ & 15 \\
\hline I oversee operator use of the RMS software data. & $18.3 \%$ & 38 \\
\hline $\begin{array}{l}\text { I review data as needed when alerted by the person } \\
\text { monitoring the RMS. }\end{array}$ & $46.3 \%$ & 14 \\
\hline Other (please specify) & $17.1 \%$ & 2 \\
\hline
\end{tabular}

\section{Comments:}

- Oversee all ARC sites with RMS workstations

- I restart/ reload/ update RMS software

- MANTAIN AND INSPECT SYSTEM

- I only ensure that the system is working

- Trouble Shoot any issues with the RMS or Assessment Computer

- Security interacts with this. I am notified if there are issues.

- I understand it to a degree

- Assist with IT-related questions

- I need to understand RMS system and use of RADEYE

- As RSO I am informed of alarms from the RMS

- Manage its operability and functionality

- Supervisor of Communications where software is installed to be monitored

- The RMS software is installed on workstation in the secure area and a LLE workstation. I have very little understanding of how it works.

- I view parallel systems on my workstation, and some video feeds.

\section{Question 12. How often do you review the RMS software data?}

\begin{tabular}{|l|c|c|}
\hline Answer Options & Response Percent & Response Count \\
\hline Never - I forget that it is there & $53.1 \%$ & 34 \\
\hline $1-5$ times per day & $42.2 \%$ & 27 \\
\hline $6-10$ times per day & $1.6 \%$ & 1 \\
\hline $10+$ times per day & $3.1 \%$ & 2 \\
\hline Additional comments & & 37 \\
\hline
\end{tabular}


Comments:

- Oversee the project not the day to day operations

- I would only review the data regarding activity alarms, otherwise security oversees all other data. If need be, I am alerted to any unusual situation.

- Have not had the opportunity to review

- I don't review it directly

- RMS software is handled by Security and IS staff members

- Once a quarter

- I only get called when there is a problem

- Primarily rely on an alarm for notification

- When the alarm goes off

- I look at the data weekly

- Not part of my normal job

- As needed, you should offer a different choice depending on the question above

- We monitor 24/7. Any data such as an alarm are handled upon activation. Any additional data monitoring is handled by a department manager ( Physical Security Manager )

- I did not forget about it

- This is good - no or very few false activations

- Occasionally

- The RMS is monitored by HUPD dispatch and I am alerted if there is an 'admin' or 'network' issue. I also use it during quarterly tests and/or for troubleshooting issues.

- Review of software data occurs only when an alarm triggers.

- Once in a while

- as above

- More like 1-2 times per month

- I don't really forget it's there, I just don't monitor it.

- I have not forgotten that it is there. Job responsibilities do not require that this is reviewed. You should reword the question so that it doesn't lead to this conclusion.

- I am rarely asked to review any of the data

- I have not reviewed RMS data system, need training.

- Quarterly performance testing

- I didn't forget, but my job doesn't have me reviewing data.

- As needed for review and QA/QC

- I review only when Security notified me with an incident

- The access is on the other side of campus

- As needed, but not very often

- Our Police Monitors RMS - we only respond

- I review the screen when I go into the communications center a few times a week.

- Only checked when there is problem or false alarm

- Just video feeds, usually left on my desktop.

- As needed

- Not applicable 


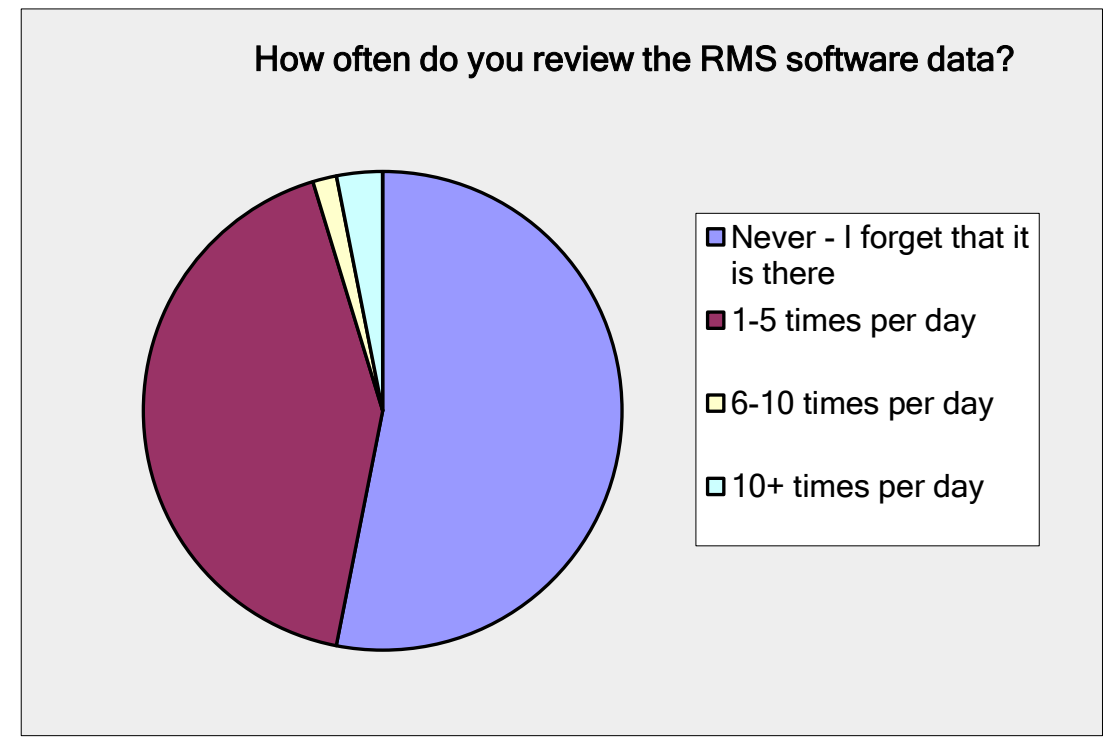

\begin{tabular}{|l|c|c|}
\hline Question 13. What percentage of your work day is spent monitoring the RMS software data? \\
\hline Answer Options & Response Percent & Response Count \\
\hline $0 \%-$ I forget it is there & $47.9 \%$ & 34 \\
\hline $1-25 \%$ & $46.5 \%$ & 33 \\
\hline $26-50 \%$ & $1.4 \%$ & 1 \\
\hline $51-75 \%$ & $1.4 \%$ & 1 \\
\hline $76-100 \%$ & $2.8 \%$ & 2 \\
\hline Additional comments & & 21 \\
\hline
\end{tabular}

\section{Comments:}

- Oversee the project not the day to day operations

- 1-2 hours / week

- I did not forget it is there, there is no need for me to review.

- I don't review it directly

- RMS software is handled by Security and IS staff members

- Once a quarter

- Minimal

- If it is running normally, only weekly for $5 \mathrm{~min}$. If they are down, I am on it until it is resolved.

- Not part of my normal job

- This is good - no or very few false activations

- It is on the low end of the above number, Dispatch monitor the software $24 \times 7$

- I don't forget it's there, I just don't actually monitor it.

- Same response as above

- Looking forward to review data

- Only when there is a problem

- I didn't forget, but I get informed on alarms and don't monitor personally.

- This is not my role

- Again, just the video feed.

- As needed 
What percentage of your work day is spent monitoring the RMS software data?

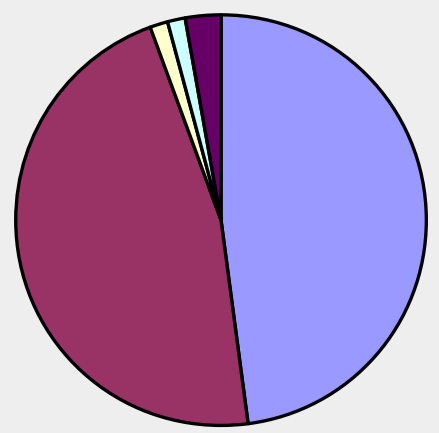

$\square 0 \%$ - I forget it is there

$\square 1-25 \%$

$\square 26-50 \%$

$\square 51-75 \%$

$76-100 \%$

Question 14. Have you received any prior RMS software training?

\begin{tabular}{|l|c|c|}
\hline Answer Options & Response Percent & Response Count \\
\hline Yes & $65.4 \%$ & 53 \\
\hline Not sure & $3.7 \%$ & 3 \\
\hline No & $30.9 \%$ & 25 \\
\hline Additional comments & & 18 \\
\hline
\end{tabular}

\section{Comments:}

- Only on site during installation but training was sufficient

- Training via installer and site visit engineers

- But it was geared towards installers.

- When initially installed and made operational

- Brief, not documented, nothing in writing - not manual

- Webinar from Aquila?

- In-service performed by installers.

- I did get an overview training when the system was initially deployed but most of my knowledge is from reading the manual and hands-on use.

- Minimal

- Alarm Response Training

- Minimal during technician's last part of install day

- I received training at the install, but not much

- $\mathrm{Y}-12$

- Hands on during installation and some at Y-12

- Minimal upon installation

- Minimal

- Brief and informal during installation

- Training received when unit was installed, no classroom training provided 


\section{Have you received any prior RMS software training?}

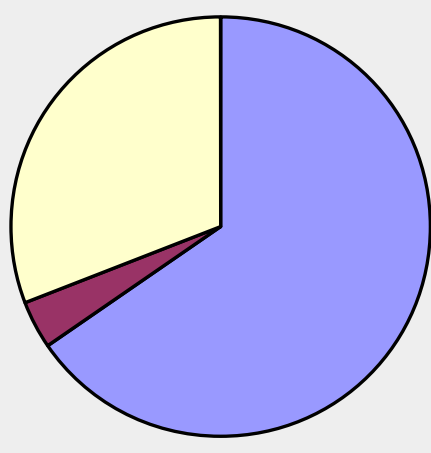

口Yes

$\square$ Not sure

$\square$ No

\section{Question 15. Was the training too easy or too complex?}

\begin{tabular}{|l|c|c|}
\hline Answer Options & Response Percent & Response Count \\
\hline Too easy & $21.8 \%$ & 12 \\
\hline Just right & $69.1 \%$ & 38 \\
\hline Too complex & $9.1 \%$ & 5 \\
\hline
\end{tabular}

Question 16. Following the instruction you received, to what extent did you feel adequately
prepared to:
\begin{tabular}{|l|c|c|c|c|c|}
\hline Answer Options & $\begin{array}{c}\text { Not at all } \\
\text { prepared }\end{array}$ & $\begin{array}{c}\text { Somewhat } \\
\text { prepared }\end{array}$ & $\begin{array}{c}\text { Well } \\
\text { prepared }\end{array}$ & $\begin{array}{c}\text { Rating } \\
\text { Average }\end{array}$ & $\begin{array}{c}\text { Response } \\
\text { Count }\end{array}$ \\
\hline Use the RMS software? & $21.7 \%$ & $42.0 \%$ & $36.2 \%$ & 2.14 & 69 \\
\hline Monitor RMS alarms? & $14.5 \%$ & $37.7 \%$ & $47.8 \%$ & 2.33 & 69 \\
\hline Understand what the alarms meant? & $7.2 \%$ & $42.0 \%$ & $50.7 \%$ & 2.43 & 69 \\
\hline $\begin{array}{l}\text { Make proper notifications when } \\
\text { receiving alarms? }\end{array}$ & $10.1 \%$ & $34.8 \%$ & $55.1 \%$ & 2.45 & 69 \\
\hline Additional comments & & & & 11 \\
\hline
\end{tabular}

Comments:

- I felt somewhat prepared, but have the impression our dispatchers are not prepared.

- A written manual is needed since multiple staff will respond to alarms. Training was brief and complex.

- These questions are dependent on the person's position and should be modified accordingly.

- I have not yet attended the online training webinar but plan to do so.

- The best training is from conducting quarterly alarm testing!

- Since I don't actually monitor alarms, I only interact with those who do monitor. However, I do not feel well prepared to troubleshoot.

- Not directly involved on a day to day basis.

- Not sure what "use the software" means. Notifications are a local event 
- One quick session. No back up if you forget something.

- I feel the RMS monitoring screen and the systems being monitored are too complicated. We have had large numbers of EMS responses to our site because of misinterpretations of the alarm screens by central station operators at the police departments. Simple loss of internet connection is resulting in a full armed response by the EMS team. It is also very cumbersome to clear events (alarms and alerts) from the screen. Some of our PD's have over 120 workers in their central stations and training them on the nuances of the system has proven difficult.

- Have not received training, yet.

\section{Question 17. If you did not feel prepared to use the RMS software, please explain why not:}

Comments:

- Don't fully understand some of the alerts

- Dispatchers monitor system

- Lack of understanding on the part of our dispatchers.

- Never trained

- The training took place a few months before system was in use. Did not remember specifics covered in training.

- No written manual. Prompts on screen are not adequate. More training - refresher is needed

- This falls under the RSO.

- Did not receive training

- The instruction was conducted for a lot of people in a very busy office. There was no one-onone training

- More training should be provided at the time of installation of the RMS software

- The training covered the main alarms but did not cover the workings of the communication between the field hardware and the assessment computer, or the details about log/history files (for reviewing old alarms, or for troubleshooting)

- Had problems with false alarms and deciphering what should be alarmed at first

- See above

- The subscribers didn't have contact lists/procedures prepared to explain how to respond/notify on alarm conditions

- Would have preferred all users had a sit-down class with demo.

- No, you forget what you learned if you don't use it daily.

- I never use it on my own

- Software is quite complicated. Would be good to have a hands-on demo with alarm scenarios. Our training was before the system was up and running so we never got to see actual alarms.

- The system needs a lot of in-house customization for the many different alerts and alarms and training in-house screen operators at the PD's has proven to be difficult.

- minimal initial training and post-install use

- Not trained, yet.

- Training could have been more thorough

- Training was very brief. No way to know if it was complete. 


\begin{tabular}{|l|c|c|}
\hline $\begin{array}{l}\text { Question 18. What other kinds of training would have helped you work with the RMS software? } \\
\text { Please select any/all that apply: }\end{array}$ & Response Percent \\
\hline Answer Options & & Response Count \\
\hline $\begin{array}{l}\text { RMS Client Software Overview \{product } \\
\text { familiarization; software overview; status level } \\
\text { setup; setting alarm conditions; tabs (alarms, } \\
\text { general, sounds)\} }\end{array}$ & $53.3 \%$ & 32 \\
\hline $\begin{array}{l}\text { Using the RMS Client Software \{simple view } \\
\text { operation; live images; polled images; latest events; } \\
\text { radiation graph; student practice\} }\end{array}$ & $46.7 \%$ & 28 \\
\hline $\begin{array}{l}\text { RMS Alarms and Related Activities \{using the alarms } \\
\text { screen; alarm viewer (polled images, latest event, } \\
\text { radiation graph); acknowledge alarm actions; alarm } \\
\text { logs; information and warning messages\} }\end{array}$ & $41.7 \%$ & 25 \\
\hline One-time Refresher Training & & \\
\hline Quarterly Refresher/New Hire Training & $16.7 \%$ & 10 \\
\hline Annual Refresher Training & $21.7 \%$ & 39 \\
\hline Other (please specify) & $65.0 \%$ & 12 \\
\hline
\end{tabular}

\section{Comments:}

- Advanced notice of refresher training and a written manual is needed

- hands on training in working environment

- N/A

- I don't deal with the system

- Having dedicated classroom training with and RMS unit and inputs etc. would have been better than the live (i.e. live system in HUPD Dispatch) training we used the initial deployment.

- A lot was given during the initial training. The personnel who provided the training did a fine job. Since my job responsibilities do not require me to interact with this on a regular basis it is not as fresh as it could be. Refresher training would be a very good idea.

- Refreshers only because we use it once a quarter for testing.

- On-call help from Aquila is sufficient

- I agree more training and refreshers are needed and should include LLE

- Something to refer to if you forget.

- Our alarm company really needs the new hire training element

- Having another client would help. 
What other kinds of training would have helped you work with the RMS software? Please select any/all that apply:

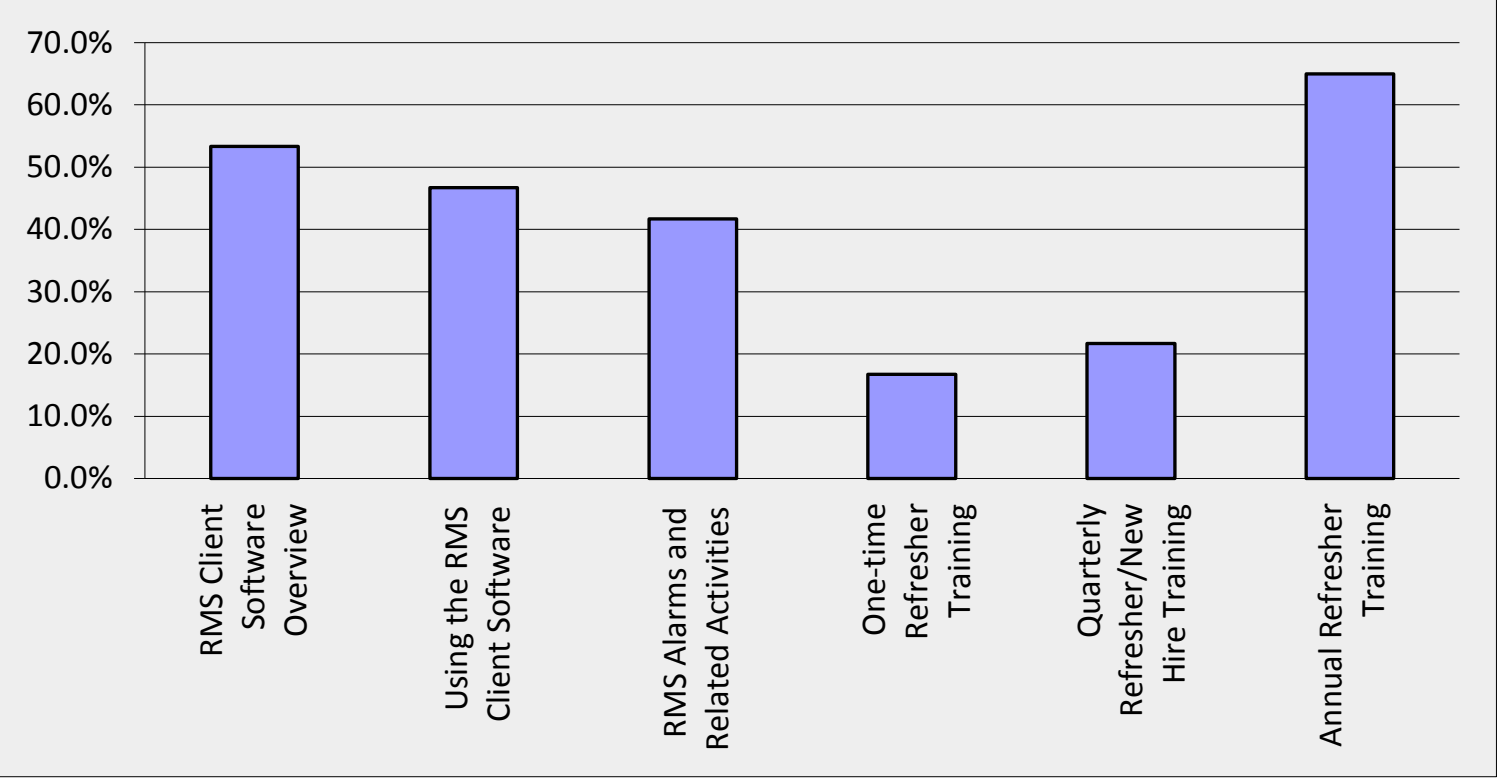

Please rate the quality of the following elements of the training you received:

\begin{tabular}{|l|c|c|c|c|c|c|c|c|}
\hline Answer Options & Poor & Fair & Good & $\begin{array}{c}\text { Very } \\
\text { Good }\end{array}$ & Excellent & $\begin{array}{c}\text { Do not } \\
\text { Recall/NA }\end{array}$ & $\begin{array}{c}\text { Rating } \\
\text { Average }\end{array}$ & $\begin{array}{c}\text { Response } \\
\text { Count }\end{array}$ \\
\hline $\begin{array}{l}\text { Overall content } \\
\text { of the training }\end{array}$ & $2.9 \%$ & $19.1 \%$ & $33.8 \%$ & $23.5 \%$ & $8.8 \%$ & $11.8 \%$ & 2.81 & 68 \\
\hline $\begin{array}{l}\text { User guide or } \\
\text { materials }\end{array}$ & $7.6 \%$ & $13.6 \%$ & $28.8 \%$ & $18.2 \%$ & $7.6 \%$ & $24.2 \%$ & 2.32 & 66 \\
\hline $\begin{array}{l}\text { Presentation of } \\
\text { material by } \\
\text { Instructor }\end{array}$ & $2.9 \%$ & $19.1 \%$ & $27.9 \%$ & $26.5 \%$ & $8.8 \%$ & $14.7 \%$ & 2.75 & 68 \\
\hline $\begin{array}{l}\text { Participant/ } \\
\text { Group activities } \\
\text { or actual practice } \\
\text { during training } \\
\text { with the RMS } \\
\text { software }\end{array}$ & $10.4 \%$ & $17.9 \%$ & $23.9 \%$ & $10.4 \%$ & $9.0 \%$ & $28.4 \%$ & 2.04 & 67 \\
\hline $\begin{array}{l}\text { Facilitation of } \\
\text { activities by } \\
\text { Instructor }\end{array}$ & $7.5 \%$ & $16.4 \%$ & $26.9 \%$ & $14.9 \%$ & $7.5 \%$ & $26.9 \%$ & 2.18 & 67 \\
\hline \begin{tabular}{l} 
Additional comments \\
\hline
\end{tabular} & & & & & & & & \\
\hline
\end{tabular}

\section{Comments:}

- Too involved with system to adequately determine

- There was no handout or manual provided.

- Onsite training

- Instructor did not seem very comfortable presenting to a group. 
- Not the instructors fault - we were unable to focus at the time due to multiple projects occurring. A written manual is required

- It's been too long since the training.

- Did not receive any training.

- NA

- The above 'poor' rating reflects the fact that we had training on our 'live' system, not in a classroom with a static/demo RMS.

- Have not received formal training

- Training was just too short in nature. Operators just got basic training, but malfunctions/false alarms are not understood.

- Again refresher training would be a good idea.

- On install we got 5 minutes of this is what it does and that was it.

- Don't recall training be this structured and/or widely offered

- Initial training was informal

- N/A: not trained

- No formal class provided, just at installation

\section{Question 20. Please share any other comments you have that would help us improve the next RMS software training.}

Comments:

- Handout material may be beneficial.

- I maintain the system. So far we have not had any trouble in the field. If something went wrong with the field equipment I would be the one they would call.

- As RSO, I am a person who does not directly read or monitor the RMS, but would get information/data from those who do monitor the RMS. I wasn't aware that my participation was needed in the initial training for the system; however, I can see the value in understanding its operation and results in order to interpret the data being provided to me. My only comment is to stress (maybe you did) who in what roles, should attend the initial training.

- Refresher training with written materials is needed

- The RMS is not hard to use. How much can you do on a webinar? Most of it is self-explanatory for reasonably computer savvy people.

- Training on how to populate alarm instructions should be provided

- Train users before deployment and not on live systems. Follow-up with some refresher training on the live system during or shortly after deployment.

- More on site Admin Rights. Maybe a Web Based Course on the Software

- Our facility needed more software training with each shift and explanation of what the alarm is. Dispatchers were confused at first with the software and what our response was to each alarm. Also updated lists of people allowed into the facilities needs to be maintained and contacts for after hours

- The dispatchers in Security would be the best personnel to answer these questions. The training for the RMS needs to be more in depth, the installer set the alarm point did a brief this is what it does and that was it.

- The software training was great. The only problem was with the notification procedure materials not being in. Because they weren't there, it wasn't really possible to walk through using the software as though we were getting an actual alarm. So I couldn't practice response and encounter any possible issues. 
- Please arrange RMS training.

- Show how to stop problems with change to daylight time.

- Some of the individuals who actually use the software and provide alarm information appear to find it easy to use while others seem to have some difficulties.

- Would volunteer to help establish training, review materials, or host a regional training. Use me if you need help.

- Break it up into small, easily literate and understandable modules, not computer language.

- Onsite training was minimal. More training would be a welcome addition to eliminate mistakes

- The RMS is kept on the other side of campus in our communications center. Having access to it remotely or having another client would help us use it more.

- The training is fine but in my opinion the display screen for the RMS is flawed. True alarms should appear with a distinct siren and flashing red color (radiation, intrusion, RFID seal etc.). Alerts should be displayed with a yellow color and a different siren, similar to how fire alarm conditions report.

- One day formal initial training and annual (2-3 hours) refresher training would be best.

\begin{tabular}{|c|c|c|c|c|c|c|}
\hline $\begin{array}{l}\text { Question 21. If you use OR n } \\
\text { the following system tasks: }\end{array}$ & itor the & S compu & lease $\epsilon$ & ate you & ent $s$ & rel with \\
\hline Answer Options & $\begin{array}{l}\text { I do not } \\
\text { perform } \\
\text { this task }\end{array}$ & $\begin{array}{c}\text { I have } \\
\text { minimal or } \\
\text { no } \\
\text { proficiency } \\
\text { at this task }\end{array}$ & $\begin{array}{l}\text { I am } \\
\text { proficient } \\
\text { at this } \\
\text { task }\end{array}$ & $\begin{array}{c}\text { I am } \\
\text { extremely } \\
\text { proficient } \\
\text { at this } \\
\text { task }\end{array}$ & $\begin{array}{l}\text { Rating } \\
\text { Average }\end{array}$ & $\begin{array}{c}\text { Response } \\
\text { Count }\end{array}$ \\
\hline $\begin{array}{l}\text { I can locate and open the } \\
\text { RMS software interface }\end{array}$ & $33.8 \%$ & $12.3 \%$ & $43.1 \%$ & $10.8 \%$ & 1.31 & 65 \\
\hline $\begin{array}{l}\text { I can locate and open the } \\
\text { RMS Viewer window }\end{array}$ & $33.8 \%$ & $9.2 \%$ & $44.6 \%$ & $12.3 \%$ & 1.35 & 65 \\
\hline $\begin{array}{l}\text { I can locate and open the } \\
\text { RMS Simple Viewer window }\end{array}$ & $35.4 \%$ & $9.2 \%$ & $43.1 \%$ & $12.3 \%$ & 1.32 & 65 \\
\hline $\begin{array}{l}\text { I can locate a specific RMS } \\
\text { device within the RMS } \\
\text { software interface }\end{array}$ & $34.8 \%$ & $15.2 \%$ & $39.4 \%$ & $10.6 \%$ & 1.26 & 66 \\
\hline $\begin{array}{l}\text { I understand the difference } \\
\text { between each status } \\
\text { indicator (good, marginal, } \\
\text { bad) }\end{array}$ & $30.8 \%$ & $18.5 \%$ & $40.0 \%$ & $10.8 \%$ & 1.31 & 65 \\
\hline $\begin{array}{l}\text { I can locate and view the } \\
\text { "Live View" }\end{array}$ & $27.7 \%$ & $6.2 \%$ & $52.3 \%$ & $13.8 \%$ & 1.52 & 65 \\
\hline $\begin{array}{l}\text { I can locate and view the } \\
\text { "Latest Events" }\end{array}$ & $28.8 \%$ & $15.2 \%$ & $43.9 \%$ & $12.1 \%$ & 1.39 & 66 \\
\hline $\begin{array}{l}\text { I can locate and view the } \\
\text { "Radiation Graph" }\end{array}$ & $25.8 \%$ & $16.7 \%$ & $45.5 \%$ & $12.1 \%$ & 1.44 & 66 \\
\hline $\begin{array}{l}\text { I can view individual graph } \\
\text { data point values }\end{array}$ & $34.4 \%$ & $20.3 \%$ & $32.8 \%$ & $12.5 \%$ & 1.23 & 64 \\
\hline $\begin{array}{l}\text { I can export the Radiation } \\
\text { Graph data }\end{array}$ & $51.6 \%$ & $29.7 \%$ & $12.5 \%$ & $6.3 \%$ & 0.73 & 64 \\
\hline I can locate and view alarm & $29.2 \%$ & $15.4 \%$ & $44.6 \%$ & $10.8 \%$ & 1.37 & 65 \\
\hline
\end{tabular}

Training and Operational Needs Assessment: Analysis Report 


\begin{tabular}{|l|c|c|c|c|c|c|}
\hline data & & & & & \\
\hline $\begin{array}{l}\text { I can locate and view alerts } \\
\text { data }\end{array}$ & $29.7 \%$ & $20.3 \%$ & $39.1 \%$ & $10.9 \%$ & 1.31 & 64 \\
\hline $\begin{array}{l}\text { I can locate and view } \\
\text { radiation readings data }\end{array}$ & $29.2 \%$ & $16.9 \%$ & $41.5 \%$ & $12.3 \%$ & 1.37 & 65 \\
\hline $\begin{array}{l}\text { I can copy data rows to } \\
\text { paste into external } \\
\text { programs }\end{array}$ & $58.7 \%$ & $25.4 \%$ & $12.7 \%$ & $3.2 \%$ & 0.60 & 63 \\
\hline $\begin{array}{l}\text { I can enlarge RMS images or } \\
\text { image sections }\end{array}$ & $43.9 \%$ & $27.3 \%$ & $22.7 \%$ & $6.1 \%$ & 0.91 & 66 \\
\hline $\begin{array}{l}\text { I can check available disk } \\
\text { storage space for the RMS } \\
\text { software data on the } \\
\text { workstation }\end{array}$ & $50.8 \%$ & $27.7 \%$ & $18.5 \%$ & $3.1 \%$ & 0.74 & 65 \\
\hline $\begin{array}{l}\text { I can check the } \\
\text { communications status } \\
\text { between each RMS and the } \\
\text { workstation }\end{array}$ & $50.8 \%$ & $20.0 \%$ & $21.5 \%$ & $7.7 \%$ & 0.86 & 65 \\
\hline $\begin{array}{l}\text { I can check the status of } \\
\text { individual RMS components }\end{array}$ & $47.7 \%$ & $27.7 \%$ & $18.5 \%$ & $6.2 \%$ & 0.83 & 65 \\
\hline $\begin{array}{l}\text { I can enable data and alarm } \\
\text { filters for each RMS }\end{array}$ & $60.9 \%$ & $26.6 \%$ & $10.9 \%$ & $1.6 \%$ & 0.53 & 64 \\
\hline Additional comments & & & 10 \\
\hline
\end{tabular}

\section{Comments:}

- I don't operate the RMS workstation as a daily functions

- I provide oversight-details managed by local observer

- We do not have the system password so cannot perform all tasks.

- The individual who managed the installation resigned his position just prior to the system going live. I feel I did not receive adequate training on the software. Additional training would be appreciated

- Clinical engineering does this job.

- I am a little unclear on some of the above questions and so have put a lower level of comfort for those items.

- Boy I really need some training after seeing this list...

- I have been working my way through the system but do not feel $100 \%$ confident that I can identify all

- The Laboratory's security department and two police departments probably have a total of 4 individuals out of over 100 that are truly proficient in the tasks described above. That is why there are a large number of mistakes made at the monitoring sites.

\section{Question 22. Software Response Tasks}

\begin{tabular}{|l|c|c|c|c|c|c|}
\hline Answer Options & $\begin{array}{c}\text { I do not } \\
\text { perform } \\
\text { this task }\end{array}$ & $\begin{array}{c}\text { I have } \\
\text { minimal or } \\
\text { no } \\
\text { proficiency } \\
\text { at this task }\end{array}$ & $\begin{array}{c}\text { I am } \\
\text { proficient } \\
\text { at this } \\
\text { task }\end{array}$ & $\begin{array}{c}\text { I am } \\
\text { extremely } \\
\text { proficient } \\
\text { at this } \\
\text { task }\end{array}$ & $\begin{array}{c}\text { Rating } \\
\text { Average }\end{array}$ & $\begin{array}{c}\text { Response } \\
\text { Count }\end{array}$ \\
\hline
\end{tabular}




\begin{tabular}{|c|c|c|c|c|c|c|}
\hline $\begin{array}{l}\text { I know how to contact } \\
\text { the System Administrator }\end{array}$ & $26.2 \%$ & $16.9 \%$ & $38.5 \%$ & $18.5 \%$ & 1.49 & 65 \\
\hline $\begin{array}{l}\text { I know when to contact } \\
\text { the System Administrator }\end{array}$ & $26.2 \%$ & $16.9 \%$ & $38.5 \%$ & $18.5 \%$ & 1.49 & 65 \\
\hline $\begin{array}{l}\text { I know how to contact } \\
\text { the GTRI RMS Help Desk }\end{array}$ & $25.0 \%$ & $21.9 \%$ & $37.5 \%$ & $15.6 \%$ & 1.44 & 64 \\
\hline $\begin{array}{l}\text { I know when to contact } \\
\text { the GTRI RMS Help Desk }\end{array}$ & $27.7 \%$ & $27.7 \%$ & $32.3 \%$ & $12.3 \%$ & 1.29 & 65 \\
\hline $\begin{array}{l}\text { I recognize the audio } \\
\text { alarm signal from the } \\
\text { monitoring workstation }\end{array}$ & $27.7 \%$ & $7.7 \%$ & $41.5 \%$ & $23.1 \%$ & 1.60 & 65 \\
\hline $\begin{array}{l}\text { I know the appropriate } \\
\text { response for a "Seal Left" } \\
\text { alert }\end{array}$ & $35.4 \%$ & $21.5 \%$ & $27.7 \%$ & $15.4 \%$ & 1.23 & 65 \\
\hline $\begin{array}{l}\text { I know the appropriate } \\
\text { response for a "Radnet } \\
\text { Trigger" alarm }\end{array}$ & $33.8 \%$ & $15.4 \%$ & $33.8 \%$ & $16.9 \%$ & 1.34 & 65 \\
\hline $\begin{array}{l}\text { I know the appropriate } \\
\text { response for a "RFID } \\
\text { Seal" alarm }\end{array}$ & $32.3 \%$ & $10.8 \%$ & $36.9 \%$ & $20.0 \%$ & 1.45 & 65 \\
\hline $\begin{array}{l}\text { I know the appropriate } \\
\text { response for a "Housing" } \\
\text { alarm }\end{array}$ & $32.8 \%$ & $12.5 \%$ & $35.9 \%$ & $18.8 \%$ & 1.41 & 64 \\
\hline $\begin{array}{l}\text { I know the appropriate } \\
\text { response for an } \\
\text { "Intrusion" alarm }\end{array}$ & $32.3 \%$ & $9.2 \%$ & $40.0 \%$ & $18.5 \%$ & 1.45 & 65 \\
\hline $\begin{array}{l}\text { I know the appropriate } \\
\text { response for a "Duress" } \\
\text { alarm }\end{array}$ & $30.8 \%$ & $9.2 \%$ & $40.0 \%$ & $20.0 \%$ & 1.49 & 65 \\
\hline $\begin{array}{l}\text { I know the appropriate } \\
\text { response for a "Trigger" } \\
\text { alarm }\end{array}$ & $33.8 \%$ & $15.4 \%$ & $35.4 \%$ & $15.4 \%$ & 1.32 & 65 \\
\hline $\begin{array}{l}\text { I know the appropriate } \\
\text { response for a "Loss of } \\
A C \text { " alarm }\end{array}$ & $32.8 \%$ & $18.8 \%$ & $32.8 \%$ & $15.6 \%$ & 1.31 & 64 \\
\hline $\begin{array}{l}\text { I know the appropriate } \\
\text { response for a "Timer" } \\
\text { alarm }\end{array}$ & $33.8 \%$ & $26.2 \%$ & $29.2 \%$ & $10.8 \%$ & 1.17 & 65 \\
\hline $\begin{array}{l}\text { I know the appropriate } \\
\text { response for a "Motion" } \\
\text { alarm }\end{array}$ & $32.3 \%$ & $9.2 \%$ & $40.0 \%$ & $18.5 \%$ & 1.45 & 65 \\
\hline $\begin{array}{l}\text { I can acknowledge } \\
\text { individual alarm events } \\
\text { that have been reviewed } \\
\text { and acted upon }\end{array}$ & $32.3 \%$ & $15.4 \%$ & $32.3 \%$ & $20.0 \%$ & 1.40 & 65 \\
\hline Additional comments & & & & & & 8 \\
\hline
\end{tabular}


Comments:

- I don't operate the RMS workstation as a daily functions

- We have not finalized the protocols for responses to events

- NA

- I am a little unclear on some of the above items (trigger, timer etc.) and so have put a lower level of comfort for those items. Due to our (I think unique design) we monitor motion and general room alarms through external systems and use the GTRI for Seal, Rad., duress and tampers

- I am the system administrator

- So when is the next class?

- The Laboratory's security department performs this function.

- Sounds like we should be invited to a course.

Questions 23-26 are voluntary identifying information. 53 respondents chose to self-identify and provide contact information for further research. Feedback was provided by individuals in the following positions:

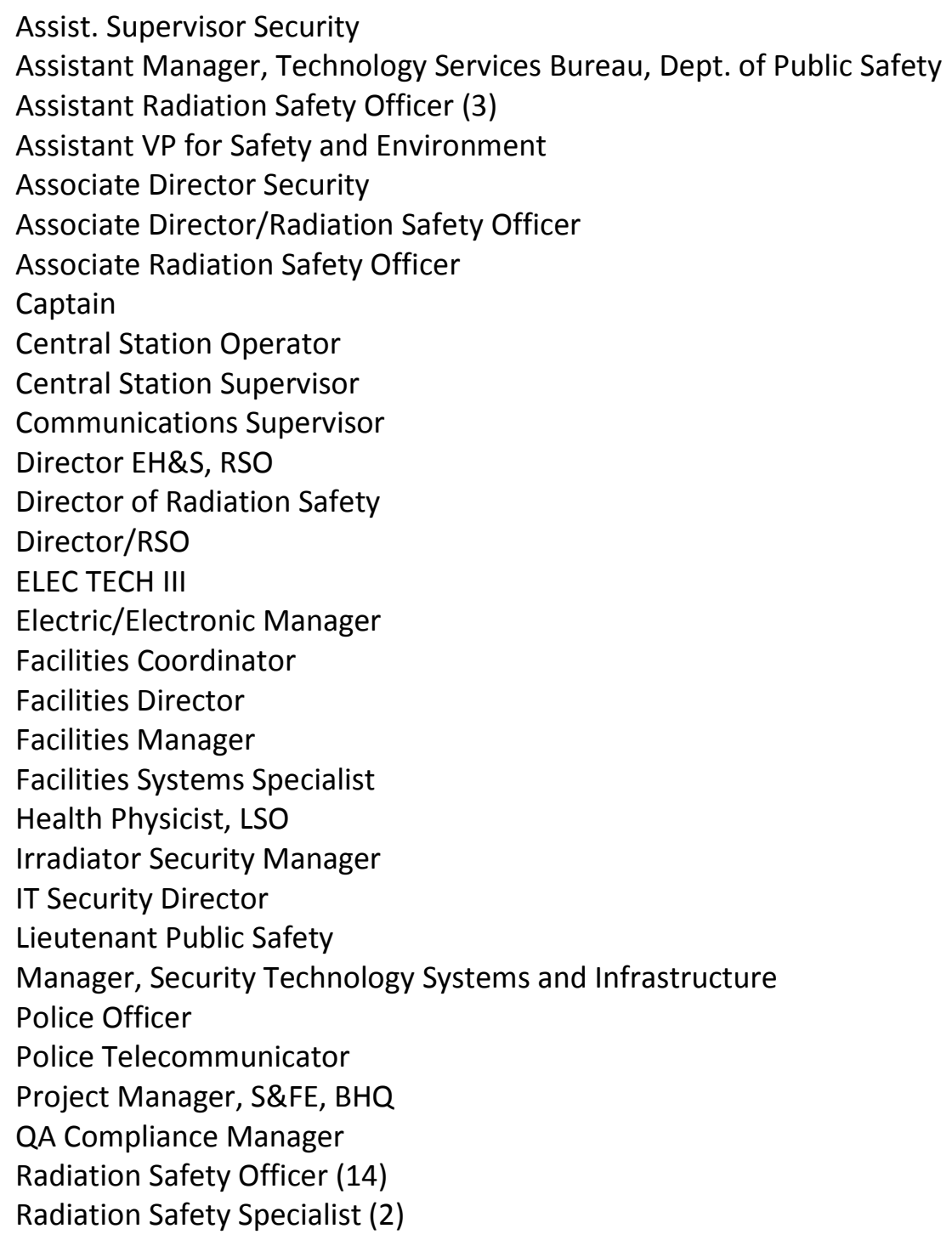


Reactor Director

RPO

Security Specialist

Security Systems BA

Senior Safety Officer

Sergeant

Training Sergeant

Return to Table of Contents 


\section{Appendix F}

\section{Operational Needs Assessment: Data Tables and Graphs}

\begin{tabular}{|l|c|c|}
\hline Question 1. In what state or U.S. territory do you live? \\
\hline Answer Options & Response Percent & Response Count \\
\hline Connecticut & $2.1 \%$ & 1 \\
\hline District of Columbia & $2.1 \%$ & 1 \\
\hline Illinois & $10.4 \%$ & 5 \\
\hline Indiana & $2.1 \%$ & 1 \\
\hline Maryland & $18.8 \%$ & 9 \\
\hline Massachusetts & $10.4 \%$ & 5 \\
\hline Mississippi & $4.2 \%$ & 2 \\
\hline Missouri & $2.1 \%$ & 1 \\
\hline New Jersey & $4.2 \%$ & 2 \\
\hline New York & $16.7 \%$ & 8 \\
\hline Pennsylvania & $8.3 \%$ & 4 \\
\hline Rhode Island & $2.1 \%$ & 1 \\
\hline Texas & $4.2 \%$ & 2 \\
\hline Virginia & $2.1 \%$ & 1 \\
\hline Washington & $8.3 \%$ & 4 \\
\hline Wisconsin & $2.1 \%$ & 1 \\
\hline
\end{tabular}

\section{Question 2. In what city do you live? \\ Response/Counts}

Brookeville

Chicago (5)

Cold Spring Harbor

College Park

Demotte

Gaithersburg

Highlands

Houston (2)

Jefferson

Kansas City

\begin{tabular}{|l|}
\hline Lowell \\
\hline McLean \\
\hline Mountlake Terrace \\
\hline Needham \\
\hline New Hyde Park \\
\hline New York (2) \\
\hline Boston \\
\hline Olney \\
\hline Philadelphia (4) \\
\hline Pleasant Prairie \\
\hline Plymouth \\
\hline Providence \\
\hline
\end{tabular}

Raymore

Rochester

Rockville (2)

Seattle (3)

Seattle

Southaven

Stony Brook

Suitland

Toms River

Wakefield

Washington, DC 


\begin{tabular}{|l|l|}
\hline \multicolumn{2}{|l|}{ Question 3. In which site or facility do you work? } \\
\hline Response/Counts & Northwest Hospital \\
\hline Baltimore & NYU Langone Medical Center \\
\hline Boston & Philadelphia \\
\hline Brown University & Puget Sound Blood Center (2) \\
\hline BTGH & Rush University Medical Center, Chicago, IL \\
\hline Children's Hospital Boston & Shady Grove Adventist Hospital \\
\hline Children's Mercy Hospital (2) & Shrewsbury \\
\hline Childrens National Medical Center & Stony Brook University \\
\hline Cold Spring Harbor Laboratory & Temple University \\
\hline Eatontown Police Department & The Methodist Hospital Research Institute \\
\hline Evanston \& Chicago & UIC \\
\hline Fox Chase Cancer Center & University of Chicago (4) \\
\hline George Washington University Hospital & University of Maryland \\
\hline Harvard University PD & University of Massachusetts Lowell \\
\hline Lifeblood - Memphis, TN & UPENN \\
\hline MIT & Vaccinex \\
\hline Mount Sinai Medical Center (2) & Washington Hospital Center \\
\hline National Institutes of Health (4) & \\
\hline NIST &
\end{tabular}

\section{Question 4. Position/primary role (choose all that apply):}

\begin{tabular}{|l|c|c|}
\hline Answer Options & Response Percent & Response Count \\
\hline Radiation Safety Officer & $34.0 \%$ & 16 \\
\hline Dispatcher & $6.4 \%$ & 3 \\
\hline Site Operator & $10.6 \%$ & 5 \\
\hline Site Administrator & $12.8 \%$ & 6 \\
\hline Site Instructor/Trainer & $14.9 \%$ & 7 \\
\hline Site Responder & $12.8 \%$ & 6 \\
\hline Offsite Responder & $2.1 \%$ & 1 \\
\hline Law Enforcement & $10.6 \%$ & 5 \\
\hline Fire Service & $0.0 \%$ & 0 \\
\hline IT Specialist & $2.1 \%$ & 1 \\
\hline Alarm Maintenance Specialist & $4.3 \%$ & 2 \\
\hline Security System Technician & $6.4 \%$ & 3 \\
\hline Site Security & $8.5 \%$ & 4 \\
\hline Security Manager/Director & $14.9 \%$ & 7 \\
\hline Assistant Security Manager/Director & $4.3 \%$ & 2 \\
\hline Other (please specify) & $17.0 \%$ & 8 \\
\hline
\end{tabular}




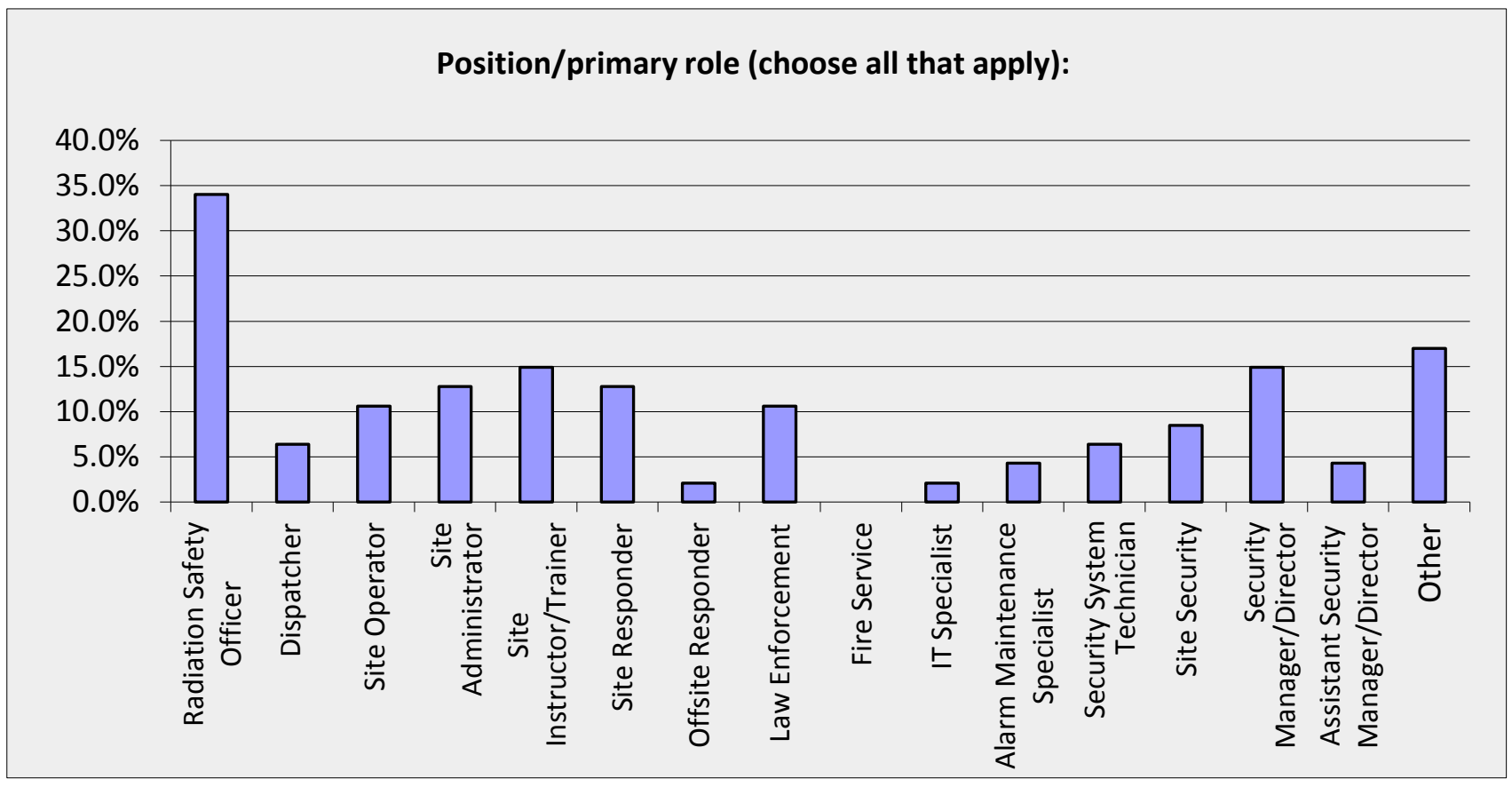

Question 5. How long has the RMS been a part of your facility's security system?

\begin{tabular}{|l|c|c|}
\hline Answer Options & Response Percent & Response Count \\
\hline Less than 1 year & $31.9 \%$ & 15 \\
\hline 1-2 years & $55.3 \%$ & 26 \\
\hline 3-4 years & $10.6 \%$ & 5 \\
\hline 5+ years & $2.1 \%$ & 1 \\
\hline Not sure & $0.0 \%$ & 0 \\
\hline Additional comments & 2 \\
\hline
\end{tabular}

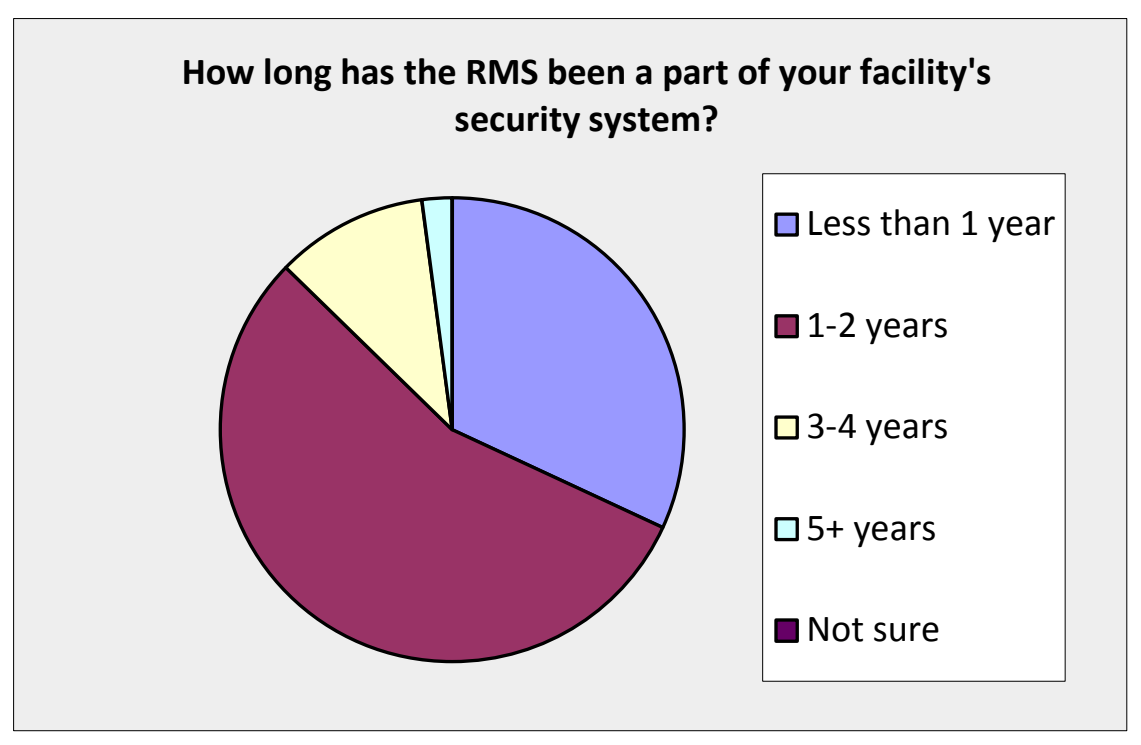




\begin{tabular}{|l|c|c|c|c|c|c|c|c|}
\hline \multicolumn{7}{|l|}{ Question 6. Indicate your overall satisfaction regarding the RMS software usability: } \\
\hline Answer Options & Poor & Fair & Good & $\begin{array}{c}\text { Very } \\
\text { Good }\end{array}$ & Excellent & $\begin{array}{c}\text { Do Not } \\
\text { Recall/ } \\
\text { NA }\end{array}$ & $\begin{array}{c}\text { Rating } \\
\text { Average }\end{array}$ & $\begin{array}{c}\text { Response } \\
\text { Count }\end{array}$ \\
\hline $\begin{array}{l}\text { Ease of monitoring } \\
\text { alarms }\end{array}$ & $0.0 \%$ & $2.6 \%$ & $28.2 \%$ & $35.9 \%$ & $30.8 \%$ & $2.6 \%$ & 3.87 & 39 \\
\hline $\begin{array}{l}\text { Ease of navigating } \\
\text { software }\end{array}$ & $0.0 \%$ & $7.9 \%$ & $34.2 \%$ & $36.8 \%$ & $13.2 \%$ & $7.9 \%$ & 3.32 & 38 \\
\hline $\begin{array}{l}\text { Ease of assessing } \\
\text { alarm and alert } \\
\text { conditions }\end{array}$ & $2.6 \%$ & $0.0 \%$ & $28.9 \%$ & $34.2 \%$ & $28.9 \%$ & $5.3 \%$ & 3.71 & 38 \\
\hline $\begin{array}{l}\text { Accessibility of data } \\
\text { logs }\end{array}$ & $0.0 \%$ & $13.2 \%$ & $23.7 \%$ & $42.1 \%$ & $10.5 \%$ & $10.5 \%$ & 3.18 & 38 \\
\hline $\begin{array}{l}\text { Overall satisfaction } \\
\text { with the RMS } \\
\text { software }\end{array}$ & $0.0 \%$ & $7.7 \%$ & $28.2 \%$ & $38.5 \%$ & $20.5 \%$ & $5.1 \%$ & 3.56 & 39 \\
\hline
\end{tabular}

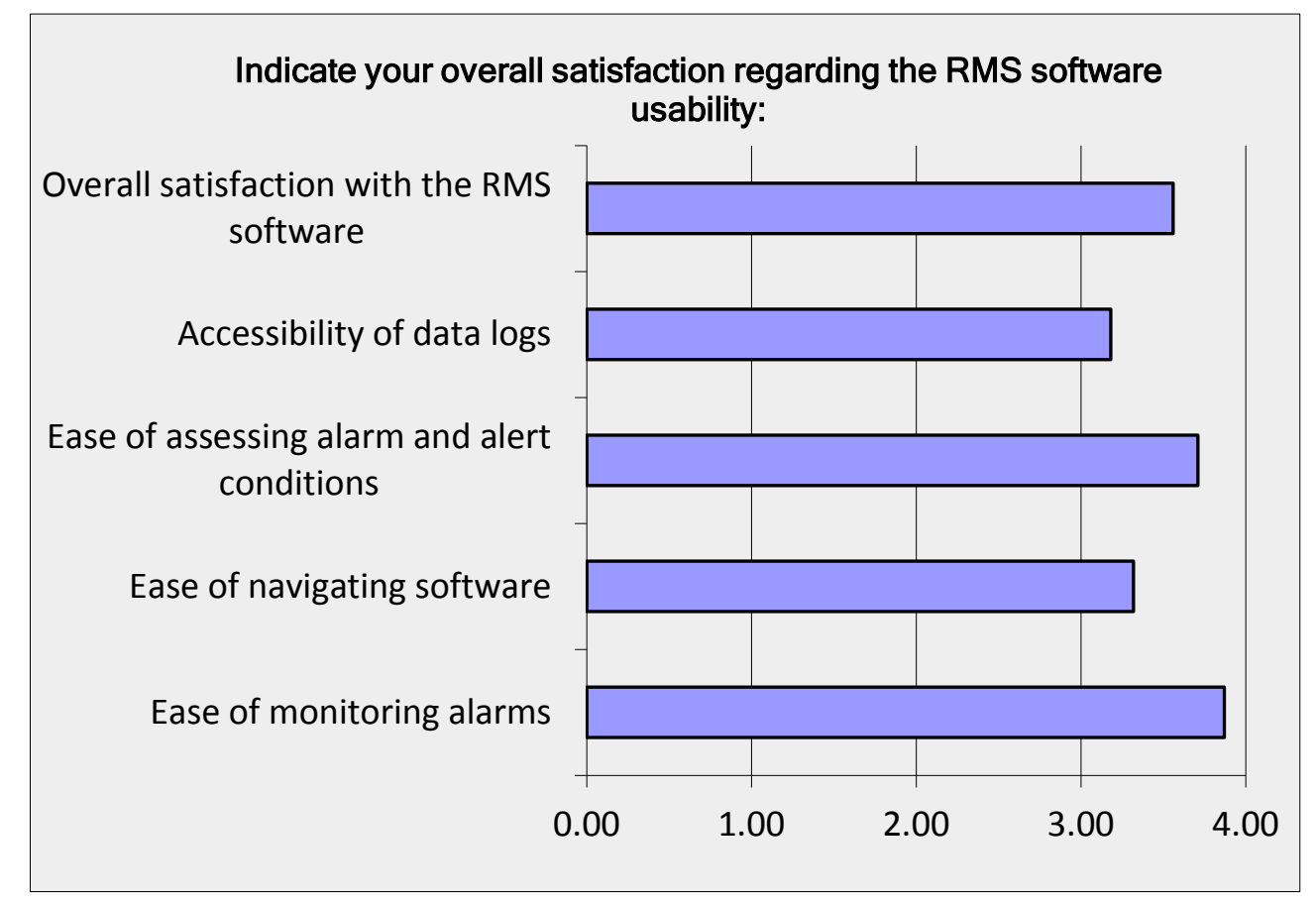

Question 7. Please provide feedback on best practices and/or potential areas for system improvement of the RMS software:

\section{Comments:}

- When an alarm occurs there is an area for comments and once comments are entered into the system we are not able to view the comments. It would help us a great deal if we were able to view all comments entered into the RMS system. 
- It does not cause any problems so I gave it a reasonable review but I have never accessed any data logs or navigated any software and no clue how to assess alarm conditions. Perhaps security does.

- It would be nice if prior alarms would be cleared from the alarms received window and banked elsewhere. No reason to see the old alarms in the window. Reduces confusion if only see current alarms.

- I've not worked with it much lately but not having the ability to export data is a weak point.

- Alarms \& Alerts should sound and appear differently at review station for more clarification.

- Provide a separate link for Radiation Safety personnel to be able to access the system either remotely or by a separate pc.

- Better initial and refresher training

- When there is certain alarm activation, a large number of alarms come in for one activation type. This takes a while for our desk officers to clear. A more 1:1 activation would be best.

- Would help tremendously if we had the system password; could access the data logs and dispatch comments then.

- With everything, there is always room for improvement. No feedback now on how to improvement the RMS software.

- The areas I believe need improvement are the following:

- Real time icons for all alarm status (power, input alarm/open-loop etc.)

- $\quad$ Battery status for the RFID tags

- $\quad$ Ability to separate the monitoring client from the database (typical IT requirement)"

- Not a primary user of the software

- Give the Facility Owner a little more Admin Rights to repair issues on our own and ensure security on a more regular basis rather than waiting for repairs.

\begin{tabular}{|c|c|c|c|c|c|c|c|c|}
\hline Answer Options & Poor & Fair & Good & $\begin{array}{l}\text { Very } \\
\text { Good }\end{array}$ & Excellent & $\begin{array}{c}\text { Do Not } \\
\text { Recall/NA }\end{array}$ & $\begin{array}{l}\text { Rating } \\
\text { Average }\end{array}$ & $\begin{array}{c}\text { Response } \\
\text { Count }\end{array}$ \\
\hline $\begin{array}{l}\text { Acceptability of } \\
\text { false alarm rate } \\
\text { (frequency of } \\
\text { incidents) }\end{array}$ & $2.6 \%$ & $17.9 \%$ & $23.1 \%$ & $28.2 \%$ & $20.5 \%$ & $7.7 \%$ & 3.23 & 39 \\
\hline $\begin{array}{l}\text { Acceptability of } \\
\text { nuisance alarm } \\
\text { rate (frequency } \\
\text { of incidents) }\end{array}$ & $0.0 \%$ & $18.4 \%$ & $15.8 \%$ & $26.3 \%$ & $28.9 \%$ & $10.0 \%$ & 3.34 & 38 \\
\hline $\begin{array}{l}\text { Quality of video } \\
\text { images }\end{array}$ & $5.1 \%$ & $5.1 \%$ & $23.1 \%$ & $38.5 \%$ & $25.6 \%$ & $2.6 \%$ & 3.67 & 39 \\
\hline $\begin{array}{l}\text { Integration with } \\
\text { house alarm } \\
\text { system }\end{array}$ & $2.6 \%$ & $2.6 \%$ & $35.9 \%$ & $35.9 \%$ & $17.9 \%$ & $5.1 \%$ & 3.49 & 39 \\
\hline $\begin{array}{l}\text { Reliability of RFID } \\
\text { seal }\end{array}$ & $2.6 \%$ & $10.3 \%$ & $23.1 \%$ & $25.6 \%$ & $33.3 \%$ & $5.1 \%$ & 3.62 & 39 \\
\hline $\begin{array}{l}\text { Reliability of } \\
\text { network } \\
\text { interface }\end{array}$ & $2.6 \%$ & $10.5 \%$ & $28.9 \%$ & $26.3 \%$ & $28.9 \%$ & $2.6 \%$ & 3.61 & 38 \\
\hline
\end{tabular}




\begin{tabular}{|l|c|c|c|c|c|c|c|c|}
\hline $\begin{array}{l}\text { Reliability of RMS } \\
\text { hardware }\end{array}$ & $0.0 \%$ & $2.7 \%$ & $35.1 \%$ & $32.4 \%$ & $21.6 \%$ & $8.1 \%$ & 3.49 & 37 \\
\hline $\begin{array}{l}\text { Ease of } \\
\text { conducting } \\
\text { maintenance } \\
\text { procedures }\end{array}$ & $0.0 \%$ & $7.9 \%$ & $26.3 \%$ & $26.3 \%$ & $7.9 \%$ & $31.6 \%$ & 2.39 & 38 \\
\hline $\begin{array}{l}\text { Quality of help } \\
\text { desk assistance }\end{array}$ & $0.0 \%$ & $2.6 \%$ & $15.4 \%$ & $25.6 \%$ & $28.2 \%$ & $28.2 \%$ & 2.95 & 39 \\
\hline $\begin{array}{l}\text { Overall stability } \\
\text { of the system }\end{array}$ & $2.6 \%$ & $2.6 \%$ & $33.3 \%$ & $30.8 \%$ & $28.2 \%$ & $2.6 \%$ & 3.72 & 39 \\
\hline $\begin{array}{l}\text { Overall } \\
\text { satisfaction with } \\
\text { RMS? }\end{array}$ & $0.0 \%$ & $5.0 \%$ & $40.0 \%$ & $27.5 \%$ & $25.0 \%$ & $2.5 \%$ & 3.65 & 40 \\
\hline
\end{tabular}

\begin{tabular}{|c|c|c|}
\hline $\begin{array}{l}\text { Question 9. In which one of the following } \\
\text { overhaul/improvement, if any? }\end{array}$ & nts/procedures wc & most like to see \\
\hline Answer Options & Response Percent & Response Count \\
\hline RMS software & $8.1 \%$ & 3 \\
\hline Data logs & $5.4 \%$ & 2 \\
\hline RFID seal & $8.1 \%$ & 3 \\
\hline Quarterly maintenance procedures & $8.1 \%$ & 3 \\
\hline RMS hardware & $0.0 \%$ & 0 \\
\hline Network interface & $2.7 \%$ & 1 \\
\hline Interface with site alarm system & $2.7 \%$ & 1 \\
\hline I am satisfied with the system as it is & $29.7 \%$ & 11 \\
\hline I do not know the system well enough yet & $27.0 \%$ & 10 \\
\hline Other & $8.1 \%$ & 3 \\
\hline If other, please specify: & & 4 \\
\hline
\end{tabular}

Comments:

- Also hardware...we did have one bad computer and one bad RFID seal

- I feel I would need more training on the RMS components/procedures to give a positive answer to the questions.

- The Seals are the primary items that need to be better but the assessment software could also be better (decouple database/logs from client monitoring, pre-defined dispatch messages)

- Integration with LLE systems 


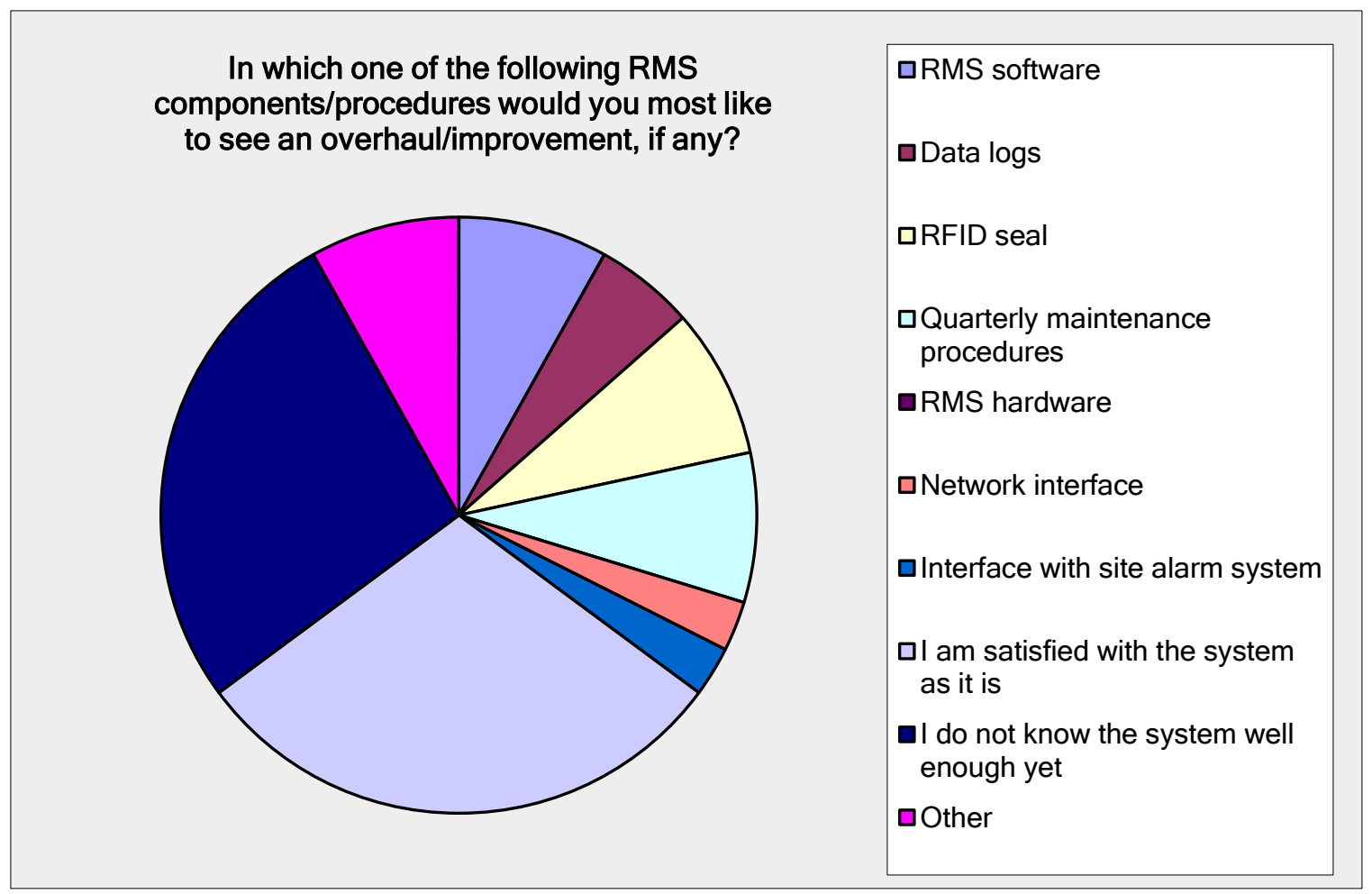

Question 10. Please provide feedback on best practices and/or potential areas for system improvement of the complete RMS:

\section{Comments:}

I think it is working okay or else security would have been complaining.

- We have had issues with network connection. We seem to have power glitches that cause the RMS to alarm, triggering a response by officers. I'm not sure if the RMS is the issue or the network/communication system.

- I would prefer that the cameras could more easily be located away from the panel.

- There should be a way to test the strobe/horn without a source (i.e. manual override).

- Alarm acknowledgment.

- We still get a fair amount of Data Broadcast Status Error alerts. Also 2 events when RFID seal failed, resulting in 30 RFID alarms per incident and necessitating seal replacement. Two events of random High Radiation Alarm (from the Ludlum problem). Video image quality is okay; surveillance camera image quality is better.

- The same as the other please specify questions, thank you.

- We had a very shaky start over about the first 18 months with the system software (v.1 crashed a few times), blown power supplies (I think we went through 3), rad-modules (I think 2), but mostly Seals (we had all seals replaced 2 or 3 times along with some wires too). I would also like if we had 3-factor authentication at the reader so we could support 'duress' entry, and also a way to support escorted access.

- RFID seals need a more sound and permanent method of attachment to units. Epoxy glue is not the best method. Better if mounted inside and out of sight

- Need to find a work around for communications loss. 
Feedback was provided by individuals in the following positions:

- Assist. Supervisor

- Assistant RSO

- Associate Director/Radiation Safety Officer

- Cad Drafter

- Captain

- Communications Supervisor

- Electric/Electronic Manager

- Facilities Coordinator

- Facilities Director

- Facilities Manager

- Health Physicist

- Irradiator Security Manager

- Manager, Security Technology Systems and Infrastructure

- Officer/Dispatcher

- Police Officer

- QA Compliance Manager

- RPO

- Radiation Safety Officer (7)

- Radiation Safety Specialist (2)

- Reactor Director

- Security Systems BA

- Supervisor Emergency Services Dispatch

Return to Table of Contents 


\section{Appendix G}

\section{Initial Survey Recipient List By Location and Position}

\begin{tabular}{|c|c|c|c|c|}
\hline State & County & Site Name & Job Title & POCs \\
\hline $\mathrm{CO}$ & Boulder & University of Colorado & $\begin{array}{l}\text { Asst. Director/Radiation Safety } \\
\text { Officer }\end{array}$ & 1 \\
\hline $\mathrm{CO}$ & Boulder & University of Colorado & Chief of Police/Executive Director & 1 \\
\hline $\mathrm{CO}$ & Denver & National Jewish Health & Radiation Safety Officer & 1 \\
\hline CT & Hartford & $\begin{array}{l}\text { University of Connecticut } \\
\text { Health Center }\end{array}$ & Assistant Radiation Safety Officer & 1 \\
\hline CT & Hartford & $\begin{array}{l}\text { University of Connecticut } \\
\text { Health Center }\end{array}$ & Radiation Safety Officer (RSO) & 1 \\
\hline CT & Hartford & $\begin{array}{l}\text { Veterans Health } \\
\text { Administration }\end{array}$ & Radiation Safety Officer & 1 \\
\hline CT & Hartford & $\begin{array}{l}\text { Veterans Health } \\
\text { Administration }\end{array}$ & Supervisory Police Officer & 1 \\
\hline DC & $\begin{array}{l}\text { District of } \\
\text { Columbia }\end{array}$ & $\begin{array}{l}\text { Children's National Medical } \\
\text { Center }\end{array}$ & Radiation Safety Officer & 1 \\
\hline DC & $\begin{array}{l}\text { District of } \\
\text { Columbia }\end{array}$ & $\begin{array}{l}\text { George Washington Univ. } \\
\text { Hospital }\end{array}$ & Head Security & 1 \\
\hline DC & $\begin{array}{l}\text { District of } \\
\text { Columbia }\end{array}$ & $\begin{array}{l}\text { George Washington University } \\
\text { Hospital }\end{array}$ & Radiation Safety Officer & 1 \\
\hline DC & $\begin{array}{l}\text { District of } \\
\text { Columbia }\end{array}$ & $\begin{array}{l}\text { Georgetown University } \\
\text { Hospital }\end{array}$ & Unknown & 1 \\
\hline DC & $\begin{array}{l}\text { District of } \\
\text { Columbia }\end{array}$ & Washington Hospital Center & Director, Radiation Safety Officer & 1 \\
\hline DC & $\begin{array}{l}\text { District of } \\
\text { Columbia }\end{array}$ & Washington Hospital Center & Unknown & 1 \\
\hline IL & Chicago & Univ. Illinois at Chicago & Assistant Director for FM & 1 \\
\hline IL & Chicago & Univ. Illinois at Chicago & Dispatcher & 1 \\
\hline IL & Chicago & Univ. Illinois at Chicago & Electrician & 1 \\
\hline $\mathrm{IL}$ & Chicago & Univ. Illinois at Chicago & Electrician Foreman & 1 \\
\hline $\mathrm{IL}$ & Chicago & Univ. Illinois at Chicago & Foreman & 2 \\
\hline IL & Chicago & Univ. Illinois at Chicago & Police & 5 \\
\hline IL & Chicago & Univ. Illinois at Chicago & Project Manager & 1 \\
\hline $\mathrm{IL}$ & Chicago & Univ. Illinois at Chicago & Security Supervisor & 1 \\
\hline $\mathrm{IL}$ & Chicago & Univ. Illinois at Chicago & UICPD & 1 \\
\hline IL & Cook & Hines VA & Criminal Investigator & 1 \\
\hline IL & Cook & Hines VA & Lieutenant & 1 \\
\hline IL & Cook & Hines VA & $\begin{array}{l}\text { Lieutenant - VA Site Police } \\
\text { Department }\end{array}$ & 1 \\
\hline IL & Cook & Hines VA & Police & 2 \\
\hline IL & Cook & Hines VA & Radiation Safety Officer (RSO) & 1 \\
\hline IL & Cook & Loyola University & $\begin{array}{l}\text { Sr. Systems Administrator and } \\
\text { Assistant Manager of Security }\end{array}$ & 1 \\
\hline
\end{tabular}




\begin{tabular}{|c|c|c|c|c|}
\hline State & County & Site Name & Job Title & POCs \\
\hline IL & Cook & $\begin{array}{l}\text { NorthShore University } \\
\text { Healthsystem }\end{array}$ & $\begin{array}{l}\text { Director and Radiation Safety } \\
\text { Officer }\end{array}$ & 1 \\
\hline IL & Cook & $\begin{array}{l}\text { NorthShore University } \\
\text { Healthsystem }\end{array}$ & Radiation Safety & 1 \\
\hline IL & Cook & Northwestern University & $\begin{array}{l}\text { Director of Emergency } \\
\text { Management }\end{array}$ & 1 \\
\hline IL & Cook & Northwestern University & Research Safety Tech NG71 & 1 \\
\hline IL & Cook & $\begin{array}{l}\text { Rush University Medical } \\
\text { Center }\end{array}$ & Electrical Manager- Rush & 1 \\
\hline IL & Cook & $\begin{array}{l}\text { Rush University Medical } \\
\text { Center }\end{array}$ & Radiation Safety Officer - Rush & 1 \\
\hline IL & Cook & University Of Chicago & $\begin{array}{l}\text { Associate Director/Radiation } \\
\text { Safety Officer }\end{array}$ & 1 \\
\hline IL & Cook & University of Chicago & Communications Supervisor & 1 \\
\hline IL & Cook & University of Chicago & Dispatcher & 2 \\
\hline IL & Cook & University of Chicago & Health Physicist & 1 \\
\hline IL & Cook & University of Chicago & Lieutenant & 1 \\
\hline IL & Cook & University Of Chicago & $\begin{array}{l}\text { Senior Director of Emergency } \\
\text { Communications and Security } \\
\text { Technology }\end{array}$ & 1 \\
\hline IL & Cook & $\begin{array}{l}\text { University Of Illinois At } \\
\text { Chicago }\end{array}$ & Radiation Safety Officer & 1 \\
\hline IL & Cook & $\begin{array}{l}\text { University Of Illinois At } \\
\text { Chicago }\end{array}$ & Senior Director of Police Services & 1 \\
\hline IN & & Purdue University & Director of Rad Laboratories & 1 \\
\hline IN & & Purdue University & Public Safety Systems Coordinator & 1 \\
\hline IN & & Purdue University & Technician & 1 \\
\hline MA & Barnstable & USDA - OTIS ANGB, MA & Laboratory Director & 1 \\
\hline MA & Barnstable & USDA - OTIS ANGB, MA & USDA Radiation Safety Officer & 1 \\
\hline $\mathrm{MA}$ & Boston & Tufts Medical Center & Operation Manager & 1 \\
\hline MA & Middlesex & Harvard University & Chief of Police & 1 \\
\hline MA & Middlesex & Harvard University & Radiation Safety Officer (RSO) & 1 \\
\hline MA & Middlesex & Harvard University & Security Systems Business Analyst & 1 \\
\hline MA & Middlesex & $\begin{array}{l}\text { Massachusetts Institute of } \\
\text { Technology }\end{array}$ & EH\&S Specialist & 1 \\
\hline MA & Middlesex & $\begin{array}{l}\text { Massachusetts Institute of } \\
\text { Technology }\end{array}$ & $\begin{array}{l}\text { MIT Radiation Protection Officer } \\
\text { (RPO) }\end{array}$ & 1 \\
\hline MA & Middlesex & $\begin{array}{l}\text { Massachusetts Institute of } \\
\text { Technology }\end{array}$ & Reactor Superintendent & 1 \\
\hline MA & Middlesex & $\begin{array}{l}\text { Massachusetts Institute of } \\
\text { Technology }\end{array}$ & Senior Safety Officer & 1 \\
\hline MA & Middlesex & $\begin{array}{l}\text { University of Massachusetts, } \\
\text { Lowell }\end{array}$ & Director, Reactor Operations & 1 \\
\hline MA & Suffolk & $\begin{array}{l}\text { Beth Israel Deaconess Medical } \\
\text { Center }\end{array}$ & Medical Health Physicist & 1 \\
\hline MA & Suffolk & $\begin{array}{l}\text { Beth Israel Deaconess Medical } \\
\text { Center }\end{array}$ & Radiation Safety Officer & 1 \\
\hline
\end{tabular}




\begin{tabular}{|c|c|c|c|c|}
\hline State & County & Site Name & Job Title & POCs \\
\hline MA & Suffolk & Children's Hospital Boston & CHB Radiation Safety Officer & 1 \\
\hline MA & Suffolk & Children's Hospital Boston & Director of Security & 1 \\
\hline MA & Suffolk & Dana-Farber Cancer Institute & Director of Security & 1 \\
\hline MA & Suffolk & Dana-Farber Cancer Institute & Radiation Safety Officer & 1 \\
\hline MA & Suffolk & Tufts Medical Center - Primary & Associate Radiation Safety Officer & 1 \\
\hline MD & Baltimore & $\begin{array}{l}\text { American Red Cross - } \\
\text { Baltimore }\end{array}$ & $\begin{array}{l}\text { Director, Facilities/Materials } \\
\text { Management }\end{array}$ & 1 \\
\hline MD & Gaithersburg & $\begin{array}{l}\text { National Institute of Standards } \\
\text { \& Technology }\end{array}$ & Console Operator & 1 \\
\hline MD & Gaithersburg & $\begin{array}{l}\text { National Institute of Standards } \\
\& \text { Technology }\end{array}$ & Dispatcher & 1 \\
\hline MD & Gaithersburg & $\begin{array}{l}\text { National Institute of Standards } \\
\& \text { Technology }\end{array}$ & Lieutenant & 1 \\
\hline MD & Gaithersburg & $\begin{array}{l}\text { National Institute of Standards } \\
\& \text { Technology }\end{array}$ & NCNR Operations & 1 \\
\hline MD & Gaithersburg & $\begin{array}{l}\text { National Institute of Standards } \\
\& \text { Technology }\end{array}$ & Security Clerk & 1 \\
\hline $\mathrm{MD}$ & Gaithersburg & PW NISTP-100 & Chief of Police & 1 \\
\hline $\mathrm{MD}$ & Gaithersburg & PW NISTP-100 & Chief, Reactor Operations & 1 \\
\hline MD & Montgomery & American Red Cross - Rockville & $\mathrm{ARC} \mathrm{BHO}$ & 1 \\
\hline MD & Montgomery & American Red Cross - Rockville & Radiation Safety Officer & 1 \\
\hline MD & Montgomery & National Institute of Health & Chief, Division of Radiation Safety & 1 \\
\hline MD & Montgomery & $\begin{array}{l}\text { National Institute of Standards } \\
\& \text { Technology }\end{array}$ & $\begin{array}{l}\text { Chief, Reactor Operations and } \\
\text { Engineering }\end{array}$ & 1 \\
\hline MD & Montgomery & $\begin{array}{l}\text { National Institute of Standards } \\
\& \text { Technology }\end{array}$ & $\begin{array}{l}\text { Radiation Safety Officer - Rad } \\
\text { Building }\end{array}$ & 1 \\
\hline MD & Montgomery & $\begin{array}{l}\text { Shady Grove Adventist } \\
\text { Hospital }\end{array}$ & Blood Bank Technical Manager & 1 \\
\hline MD & Montgomery & $\begin{array}{l}\text { Shady Grove Adventist } \\
\text { Hospital }\end{array}$ & Site Contract/Procurement POC & 1 \\
\hline MD & Montgomery & $\begin{array}{l}\text { Uniformed Services University } \\
\text { of Health Sciences }\end{array}$ & Assistant Vice President for ESH & 1 \\
\hline MD & Montgomery & $\begin{array}{l}\text { Uniformed Services University } \\
\text { of Health Sciences }\end{array}$ & Security & 1 \\
\hline MD & $\begin{array}{l}\text { Prince } \\
\text { George's }\end{array}$ & $\begin{array}{l}\text { University of Maryland College } \\
\text { Park }\end{array}$ & $\begin{array}{l}\text { Assistant Director, Radiation } \\
\text { Safety Officer }\end{array}$ & 1 \\
\hline MD & $\begin{array}{l}\text { Prince } \\
\text { George's }\end{array}$ & $\begin{array}{l}\text { University of Maryland College } \\
\text { Park }\end{array}$ & Major & 1 \\
\hline $\mathrm{MO}$ & Jackson & Children's Mercy Hospital & Assistant Director of Security & 1 \\
\hline $\mathrm{MO}$ & Jackson & Children's Mercy Hospital & Radiation Safety Officer & 1 \\
\hline MO & Jackson & St. Luke's Healthcare & $\begin{array}{l}\text { Radiation Safety Officer - Saint } \\
\text { Luke's Health Sys }\end{array}$ & 1 \\
\hline $\mathrm{MO}$ & Kansas City & Children's Mercy Hospital & Administrative Assistant & 2 \\
\hline $\mathrm{MO}$ & Kansas City & Children's Mercy Hospital & Blood Bank Supervisor & 1 \\
\hline $\mathrm{MO}$ & Kansas City & Children's Mercy Hospital & Office Manager & 1 \\
\hline $\mathrm{MO}$ & Kansas City & Children's Mercy Hospital & Project Manager & 1 \\
\hline $\mathrm{MO}$ & Kansas City & Children's Mercy Hospital & Security & 1 \\
\hline
\end{tabular}




\begin{tabular}{|c|c|c|c|c|}
\hline State & County & Site Name & Job Title & POCs \\
\hline $\mathrm{MO}$ & Kansas City & Children's Mercy Hospital & Security Assistant Supervisor & 1 \\
\hline $\mathrm{MO}$ & Kansas City & Children's Mercy Hospital & Security Supervisor & 1 \\
\hline $\mathrm{MO}$ & Kansas City & Children's Mercy Hospital & Unknown & 1 \\
\hline MT & Missoula & $\begin{array}{l}\text { Providence Saint Patrick } \\
\text { Hospital }\end{array}$ & $\mathrm{CSO}$ & 6 \\
\hline MT & Missoula & $\begin{array}{l}\text { Providence Saint Patrick } \\
\text { Hospital }\end{array}$ & PBX & 1 \\
\hline MT & Missoula & ST. PATRICK HOSPITAL & $\begin{array}{l}\text { Director Safety \& Emergency } \\
\text { Preparedness }\end{array}$ & 1 \\
\hline MT & Missoula & ST. PATRICK HOSPITAL & Radiation Safety Officer & 1 \\
\hline MT & Ravalli & NIH Rocky Mountain Labs & Captain NIH Police & 1 \\
\hline MT & Ravalli & NIH Rocky Mountain Labs & Engineer Technician & 1 \\
\hline MT & Ravalli & NIH Rocky Mountain Labs & Health Physicist & 1 \\
\hline MT & Ravalli & NIH Rocky Mountain Labs & IT Specialist & 1 \\
\hline MT & Ravalli & NIH Rocky Mountain Labs & IT Support Analyst & 1 \\
\hline MT & Ravalli & NIH Rocky Mountain Labs & Maintenance & 1 \\
\hline MT & Ravalli & NIH Rocky Mountain Labs & $\begin{array}{l}\text { Radiation Safety \& Environmental } \\
\text { Compliance }\end{array}$ & 1 \\
\hline MT & Ravalli & NIH Rocky Mountain Labs & Sergeant NIH Police & 1 \\
\hline NJ & Eatontown & Central Jersey Blood Center & IT Coordinator, EPD & 1 \\
\hline NJ & Middlesex & St. Peter's University Hospital & Medical Physicist & 1 \\
\hline NJ & Monmouth & Central Jersey Blood Center & Facilities Coordinator & 1 \\
\hline NJ & & $\begin{array}{l}\text { Saint Peter's University } \\
\text { Hospital }\end{array}$ & Assistant Director Safety/Security & 1 \\
\hline NJ & & $\begin{array}{l}\text { Saint Peter's University } \\
\text { Hospital }\end{array}$ & Coordinator Safety \& Security & 1 \\
\hline NJ & & $\begin{array}{l}\text { Saint Peter's University } \\
\text { Hospital }\end{array}$ & Director Safety \& Security & 1 \\
\hline NJ & & $\begin{array}{l}\text { Saint Peter's University } \\
\text { Hospital }\end{array}$ & Program Manager & 1 \\
\hline NJ & & $\begin{array}{l}\text { Saint Peter's University } \\
\text { Hospital }\end{array}$ & Radiation Safety Officer & 1 \\
\hline NY & Albany & Albany Medical Center & Assistant Radiation Safety Officer & 1 \\
\hline NY & Albany & Albany Medical Center & Radiation Safety Officer (RSO) & 1 \\
\hline NY & Bronx & Montefiore Medical Center & $\begin{array}{l}\text { Radiation Safety Officer - } \\
\text { Montefiore }\end{array}$ & 1 \\
\hline NY & Bronx & Montefiore Medical Center & Security Manager - Moses & 1 \\
\hline NY & Bronx & Montefiore Medical Center & Security Manager - North & 1 \\
\hline NY & Monroe & University of Rochester & Radiation Safety Officer & 1 \\
\hline NY & Monroe & Vaccinex & Facility Manager & 1 \\
\hline NY & Monroe & Vaccinex & $\begin{array}{l}\text { Radiation Safety Officer and Lab } \\
\text { Manager }\end{array}$ & 1 \\
\hline NY & Nassau & Cold Spring Harbor Laboratory & $\begin{array}{l}\text { Director Environmental Health } \\
\text { and Safety }\end{array}$ & 1 \\
\hline NY & Nassau & Cold Spring Harbor Laboratory & Security & 1 \\
\hline NY & Nassau & Unknown & Project Manager & 1 \\
\hline NY & Nassau & Unknown & Technician & 2 \\
\hline
\end{tabular}




\begin{tabular}{|c|c|c|c|c|}
\hline State & County & Site Name & Job Title & POCs \\
\hline NY & New York & Bellevue Hospital & $\begin{array}{l}\text { Assistant Director of Hospital } \\
\text { Police }\end{array}$ & 1 \\
\hline NY & New York & Columbia University & Assistant Radiation Safety Officer & 1 \\
\hline NY & New York & Columbia University & Associate Vice President & 1 \\
\hline NY & New York & Columbia University & $\begin{array}{l}\text { Radiation Safety Officer - CUMC } \\
\text { and NY Presbyterian }\end{array}$ & 1 \\
\hline NY & New York & Columbia University & Unknown & 9 \\
\hline NY & New York & Lenox Hill Hospital & Director, Security Services & 1 \\
\hline NY & New York & Mt Sinai Medical Center & Radiation Safety Officer - Mt Sinai & 1 \\
\hline NY & New York & New York University & Radiation Safety Officer & 1 \\
\hline NY & New York & New York University & Radiation Safety Officer & 1 \\
\hline NY & New York & NY Presbyterian Hospital & $\begin{array}{l}\text { Manager of Operations- NY } \\
\text { Presbyterian }\end{array}$ & 1 \\
\hline NY & New York & NY Presbyterian Hospital & Security & 2 \\
\hline NY & New York & NY Presbyterian Hospital & Sergeant & 3 \\
\hline NY & New York & NY Presbyterian Hospital & Unknown & 1 \\
\hline NY & New York & Rockefeller University & Director of Security & 1 \\
\hline NY & New York & Rockefeller University & Radiation Safety Officer & 1 \\
\hline NY & $\begin{array}{l}\text { New York } \\
\text { City }\end{array}$ & NYU Langone Medical Center & Operation Manager & 1 \\
\hline NY & $\begin{array}{l}\text { New York } \\
\text { City }\end{array}$ & NYU Langone Medical Center & Security Manager & 1 \\
\hline NY & $\begin{array}{l}\text { New York } \\
\text { City }\end{array}$ & NYU Langone Medical Center & Security Supervisor & 1 \\
\hline NY & $\begin{array}{l}\text { New York } \\
\text { City }\end{array}$ & NYU Langone Medical Center & Training Manager & 1 \\
\hline NY & Rochester & Vaccinex & Casco Security & 1 \\
\hline NY & Rochester & Vaccinex & Central Station & 2 \\
\hline NY & Rochester & Vaccinex & Operation Manager & 1 \\
\hline NY & Rochester & Vaccinex & Unknown & 1 \\
\hline NY & Suffolk & Stony Brook University & Radiation Safety Officer & 1 \\
\hline NY & Suffolk & Stony Brook University & Staff Assistant, University Police & 1 \\
\hline NY & Westchester & Westchester Medical Center & Director of Security & 1 \\
\hline NY & Westchester & Westchester Medical Center & Radiation Safety Officer & 1 \\
\hline NY & & Rockefeller University & Radiation Safety Officer & 2 \\
\hline NY & & Rockefeller University & Security & 1 \\
\hline NY & & Rockefeller University & Unknown & 1 \\
\hline $\mathrm{OH}$ & Hamilton & University of Cincinnati & Assistant Radiation Safety & 1 \\
\hline $\mathrm{OH}$ & Hamilton & University of Cincinnati & Radiation Safety Officer & 1 \\
\hline $\mathrm{PA}$ & Centre & Penn State University & Radiation Safety Officer & 1 \\
\hline PA & Centre & Penn State University & Reactor Director & 1 \\
\hline $\mathrm{PA}$ & Centre & Penn State University & Reactor Supervisor & 1 \\
\hline PA & Montgomery & Glaxo Smith Kline & $\begin{array}{l}\text { Radiation Safety Officer (RSO) - } \\
\text { GSK }\end{array}$ & 1 \\
\hline $\mathrm{PA}$ & Montgomery & Merck & Corporate Radiation Safety Officer & 1 \\
\hline $\mathrm{PA}$ & Montgomery & Merck & Health Physicist & 1 \\
\hline $\mathrm{PA}$ & Montour & Geisinger Health System & Director, Security and Emergency & 1 \\
\hline
\end{tabular}

Training and Operational Needs Assessment: Analysis Report 


\begin{tabular}{|c|c|c|c|c|}
\hline State & County & Site Name & Job Title & POCs \\
\hline PA & Philadelphia & $\begin{array}{l}\text { American Red Cross: } \\
\text { Philadelphia }\end{array}$ & $\begin{array}{l}\text { Real Estate and Fixed Assets } \\
\text { Management }\end{array}$ & 1 \\
\hline $\mathrm{PA}$ & Philadelphia & Drexel University & Radiation Safety Officer (RSO) & 1 \\
\hline $\mathrm{PA}$ & Philadelphia & Fox Chase Cancer Center & Director of Security & 1 \\
\hline PA & Philadelphia & Fox Chase Cancer Center & Radiation Safety Officer (RSO) & 1 \\
\hline PA & Philadelphia & $\begin{array}{l}\text { Hahnemann University } \\
\text { Hospital }\end{array}$ & $\begin{array}{l}\text { Radiation Safety Officer - } \\
\text { Hahnemann }\end{array}$ & 1 \\
\hline PA & Philadelphia & $\begin{array}{l}\text { Hahnemann University } \\
\text { Hospital }\end{array}$ & Security Manager & 1 \\
\hline PA & Philadelphia & St. Christopher's Hospital & $\begin{array}{l}\text { Director of Administrative and } \\
\text { Technical Services }\end{array}$ & 1 \\
\hline $\mathrm{PA}$ & Philadelphia & St. Christopher's Hospital & Director of Support Services & 1 \\
\hline PA & Philadelphia & Temple University & $\begin{array}{l}\text { Radiation Safety Officer (RSO) - } \\
\text { Temple University }\end{array}$ & 1 \\
\hline PA & Philadelphia & Thomas Jefferson University & $\begin{array}{l}\text { Radiation Safety Officer -Thomas } \\
\text { Jefferson University }\end{array}$ & 1 \\
\hline PA & Philadelphia & Thomas Jefferson University & $\begin{array}{l}\text { Senior Project Manager - Siemens } \\
\text { Industry, Inc. }\end{array}$ & 1 \\
\hline PA & Philadelphia & University Of Pennsylvania & $\begin{array}{l}\text { Radiation Safety Officer - } \\
\text { University of Pennsylvania }\end{array}$ & 1 \\
\hline $\mathrm{RI}$ & Providence & Brown University & Associate Director of Facilities & 1 \\
\hline $\mathrm{RI}$ & Providence & Brown University & Card Access Controller & 1 \\
\hline $\mathrm{RI}$ & Providence & Brown University & Commander of Police Department & 1 \\
\hline $\mathrm{RI}$ & Providence & Brown University & Communications Control Officer & 1 \\
\hline $\mathrm{RI}$ & Providence & Brown University & Crime Analyst & 1 \\
\hline $\mathrm{RI}$ & Providence & Brown University & Director of Facilities & 1 \\
\hline $\mathrm{RI}$ & Providence & Brown University & Public Safety Officer & 2 \\
\hline $\mathrm{RI}$ & Providence & Brown University & Radiation Safety Officer & 1 \\
\hline $\mathrm{RI}$ & Providence & Brown University & Radiation Safety Specialist & 1 \\
\hline $\mathrm{RI}$ & Providence & Brown University & Sergeant & 1 \\
\hline TN & Shelby & Life Blood & Radiation Safety Officer & 1 \\
\hline TN & Shelby & $\begin{array}{l}\text { Methodist Healthcare } \\
\text { University Hospital - TN }\end{array}$ & Director of Security & 1 \\
\hline TN & Shelby & $\begin{array}{l}\text { St. Jude Children's Research } \\
\text { Hospital }\end{array}$ & Security Director & 1 \\
\hline TX & Bexar & $\begin{array}{l}\text { University of Texas Health } \\
\text { Science Center }\end{array}$ & Radiation Safety Officer & 1 \\
\hline TX & Harris & Baylor College of Medicine & $\begin{array}{l}\text { Director, BCM Office of } \\
\text { Environmental Safety }\end{array}$ & 1 \\
\hline $\mathrm{TX}$ & Harris & Ben Taub General Hospital & Medical Physicist & 1 \\
\hline $\mathrm{TX}$ & Harris & Ben Taub General Hospital & Supervisor & 1 \\
\hline $\mathrm{TX}$ & Harris & Memorial Hermann Hospital & Radiation Safety Officer & 1 \\
\hline TX & Harris & Methodist University Hospital & Call Center Coordinator & 1 \\
\hline$T X$ & Harris & Methodist University Hospital & Radiation Safety Officer & 1 \\
\hline WA & King & $\begin{array}{l}\text { Benaroya Research Institute at } \\
\text { Virginia Mason }\end{array}$ & Unknown & 2 \\
\hline WA & King & Boeing & Radiation Safety Officer & 1 \\
\hline
\end{tabular}




\begin{tabular}{|l|l|l|l|l|}
\hline State & \multicolumn{1}{|c|}{ County } & \multicolumn{1}{|c|}{ Job Title Name } & POCs \\
\hline WA & King & $\begin{array}{l}\text { NW HOSPITAL GAMMA KNIFE } \\
\text { CENTER }\end{array}$ & Facilities Manager & 1 \\
\hline WA & King & $\begin{array}{l}\text { NW HOSPITAL GAMMA KNIFE } \\
\text { CENTER }\end{array}$ & Radiation Safety Officer & 1 \\
\hline WA & King & $\begin{array}{l}\text { NW HOSPITAL GAMMA KNIFE } \\
\text { CENTER }\end{array}$ & Unknown & 1 \\
\hline WA & King & University of Washington & Radiation Safety Officer-UW & 1 \\
\hline WA & Pierce & $\begin{array}{l}\text { TACOMA-PIERCE COUNTY } \\
\text { BLOOD BANK }\end{array}$ & Director of Laboratory Operations & 1 \\
\hline WA & Pierce & $\begin{array}{l}\text { TACOMA-PIERCE COUNTY } \\
\text { BLOOD BANK }\end{array}$ & Radiation Safety Officer & 1 \\
\hline WA & Seattle & Harborview & Security Supervisor & 1 \\
\hline WA & Seattle & PSBC Terry Ave & Biomed & 1 \\
\hline WA & Seattle & PSBC Terry Ave & Chief Engineer Facilities & 1 \\
\hline WA & Seattle & PSBC Terry Ave & Director Facilities & 1 \\
\hline WA & Seattle & PSBC Terry Ave & $\begin{array}{l}\text { Facilities and Engineering } \\
\text { Supervisor }\end{array}$ & 1 \\
\hline WA & Seattle & PSBC Terry Ave & Facilities Systems Specialist & 1 \\
\hline WA & Seattle & PSBC Terry Ave & Quality Coordinator & 1 \\
\hline WA & Seattle & $\begin{array}{l}\text { Univ. of WA Health Sciences } \\
\text { Ctr. }\end{array}$ & Unknown & 3 \\
\hline WA & Seattle & Univ. of WA Medical Center & CS Supervisor & 1 \\
\hline
\end{tabular}

Return to Table of Contents 


\section{Appendix $\mathbf{H}$}

\section{Glossary of Evaluation Analysis Terms}

\begin{tabular}{|c|c|}
\hline Cleaning Data & $\begin{array}{l}\text { The process of excluding from analysis forms or individual responses } \\
\text { that are substantially incomplete, or do not make sense. }\end{array}$ \\
\hline Coding Instruments & The process of converting responses to numbers for data entry. \\
\hline Content Analysis & $\begin{array}{l}\text { The process used to organize open-ended, unstructured information } \\
\text { (qualitative data). }\end{array}$ \\
\hline Data Analysis & $\begin{array}{l}\text { The process of putting together qualitative and/or quantitative } \\
\text { information to derive answers to questions. }\end{array}$ \\
\hline Database & $\begin{array}{l}\text { Computer software that can be used for data entry, analysis and } \\
\text { retrieval. Data is entered line by line, where each line contains the } \\
\text { information for one filled instrument. }\end{array}$ \\
\hline Emergent Categories & Categories in a content analysis determined after reading the raw data. \\
\hline Frequency & The number of times a given response occurs. \\
\hline Instrument & $\begin{array}{l}\text { A device for recording, indicating, measuring or collecting information } \\
\text { to measure your objective. }\end{array}$ \\
\hline Mean & $\begin{array}{l}\text { The average of numeric responses or scores. This is obtained by } \\
\text { dividing the total of all responses to an item by the number of } \\
\text { responses obtained. }\end{array}$ \\
\hline Measure & $\begin{array}{l}\text { (Verb) The process of counting information. (Noun) the instrument } \\
\text { used to quantify information. }\end{array}$ \\
\hline Percent Distribution & The proportion of respondents selecting each response. \\
\hline Pre/Post Test & $\begin{array}{l}\text { An instrument administered at two or more points in time, used to } \\
\text { capture gains over that period of time. }\end{array}$ \\
\hline Predetermined Categories & Categories in a content analysis determined before data is collected. \\
\hline Qualitative Data & $\begin{array}{l}\text { Data that comes from open-ended questions, in the form of words. } \\
\text { These may be written or recorded. }\end{array}$ \\
\hline Quantitative Data & $\begin{array}{l}\text { Data which are provided in the form of numbers or can be converted } \\
\text { to numeric form This type of data usually comes from structured } \\
\text { instrument where respondents are required to select a response or } \\
\text { provide a numeric response. This may also include test scores. }\end{array}$ \\
\hline Raw data & $\begin{array}{l}\text { All of the data collected from the instruments before they are } \\
\text { analyzed. }\end{array}$ \\
\hline Response & The individual answer selected or given to a question. \\
\hline Spreadsheet & $\begin{array}{l}\text { Computer software that allows data arranged in a grid format. Allows } \\
\text { for east data entry and basic analysis. }\end{array}$ \\
\hline
\end{tabular}


This page intentionally blank 


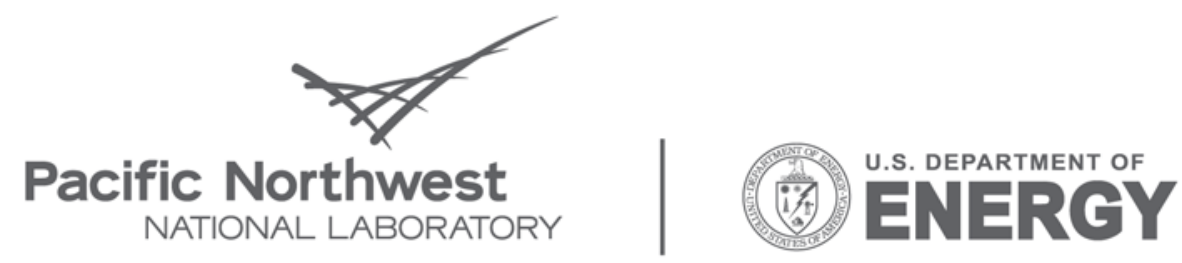

Proudly Operated by Battelle Since 1965

902 Battelle Boulevard

P.O. Box 999

Richland, WA 99352

1-888-375-PNNL (7665)

www.pnl.gov 